\title{
WestVirginiaUniversity
}

THE RESEARCH REPOSITORY @ WVU

Graduate Theses, Dissertations, and Problem Reports

2005

\section{Laser-induced breakdown spectroscopy as a diagnostic tool for coal fines}

\author{
I. Andrew Aurelio \\ West Virginia University
}

Follow this and additional works at: https://researchrepository.wvu.edu/etd

\section{Recommended Citation}

Aurelio, I. Andrew, "Laser-induced breakdown spectroscopy as a diagnostic tool for coal fines" (2005). Graduate Theses, Dissertations, and Problem Reports. 1653.

https://researchrepository.wvu.edu/etd/1653

This Thesis is protected by copyright and/or related rights. It has been brought to you by the The Research Repository @ WVU with permission from the rights-holder(s). You are free to use this Thesis in any way that is permitted by the copyright and related rights legislation that applies to your use. For other uses you must obtain permission from the rights-holder(s) directly, unless additional rights are indicated by a Creative Commons license in the record and/ or on the work itself. This Thesis has been accepted for inclusion in WVU Graduate Theses, Dissertations, and Problem Reports collection by an authorized administrator of The Research Repository @ WVU. For more information, please contact researchrepository@mail.wvu.edu. 


\title{
Laser-Induced Breakdown Spectroscopy as a Diagnostic Tool for Coal Fines
}

\author{
I. Andrew Aurelio \\ Thesis submitted to the College of Engineering and Mineral Resources \\ at West Virginia University \\ in partial fulfillment of the requirements for the degree of
}

\author{
Master of Science \\ in \\ Mechanical Engineering
}

Bruce Kang, Ph.D., Chair

Eric Johnson, Ph.D.

Steven Woodruff, Ph.D.

Department of Mechanical and Aerospace Engineering

\section{Morgantown, West Virginia}

2005

Keywords: LIBS, Coal, Laser-Spark, Time-Resolved 


\section{ABSTRACT \\ Laser-Induced Breakdown Spectroscopy as a Diagnostic Tool for Coal Fines}

\section{Andrew Aurelio}

Laser Induced Breakdown Spectroscopy (LIBS) is a technique that uses a laser, to focus down and atomize a sample of desired material. Focusing of the laser onto the material causes a plasma formation, which the material is broken down into excited ionic and atomic states. The atoms then emit characteristic optical radiation. Collection of the emitted light can be used to provide information on the elemental composition of the material. This research investigates a fundamental study of laser-induced breakdown spectroscopy (LIBS) applied to coal samples, coal fines, and fly ash. During this research, apparatus and methodology were developed to quantify the content of carbon, sulfur, iron and mercury in coal. It was observed carbon and mercury could be quantified using LIBS. A polygonal scanning mirror was added to the LIBS apparatus to observe lifetimes of emission lines. The data showed that each emission line showed different time dependent characteristics within the laser spark. 


\section{ACKNOWLEDGEMENTS}

I would like express my deepest gratitude toward Dr. Bruce Kang for the opportunity to work for him, and for his guidance, patience, and technical advice throughout this research. I would also like to thank Dr. Steven Woodruff and Dr. Eric Johnson for their guidance, patience and advice.

I would also like to thank Cliff Judy for machining critical instrumentation in the apparatus, Chuck Coleman and Carol Wells for their quick work with environmental health and safety, Dr. Peter Stansberry and Albert Taylor for the temporary use of their measuring and grinding equipment, and Dustin McIntyre and Dr. Chuanyu Feng for their technical advice and support.

Finally, I would like to thank my wife, Kimberly Aurelio, my parents, Ike and Shauna Aurelio and my former professor, Dr. Albert Popson for their moral support and encouragement throughout my studies in graduate school. 


\section{TABLE OF CONTENTS}

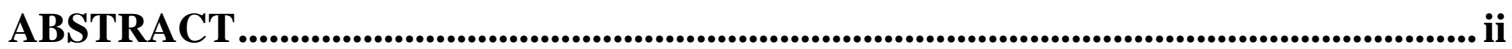

ACKNOWLEDGEMENTS ....................................................................................................ii

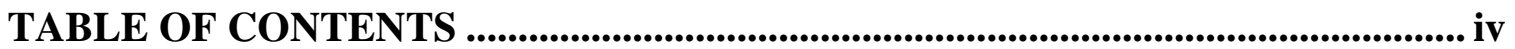

LIST OF TALBES

LIST OF FIGURES ................................................................................................................. viii

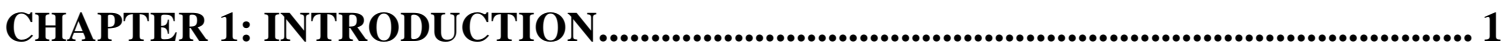

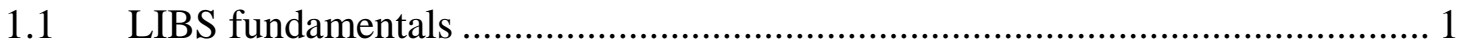

1.2 Research Objective .............................................................................................. 4

CHAPTER 2: LITERATURE REVIEW............................................................................ 6

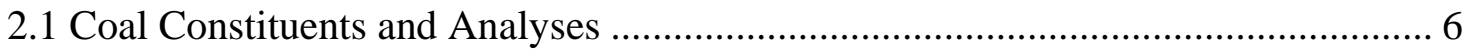

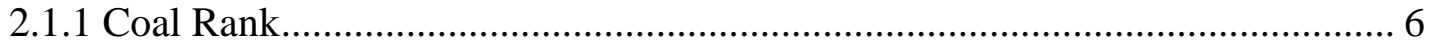

2.1.2 Coal Analyses …………………................................................................... 7

2.1.3 Coal Constituents ..................................................................................... 9

2.2 Experiments of LIBS applied to coal.............................................................. 10

2.3 Experiments of LIBS on Elements of Interest ...................................................... 14

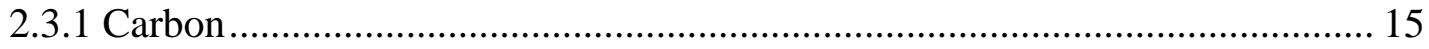

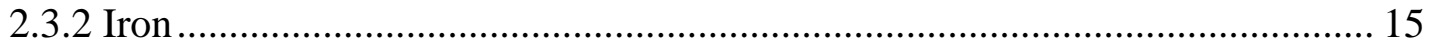

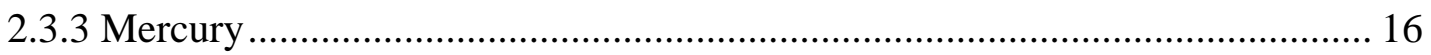

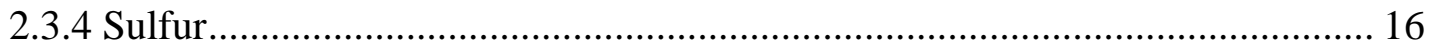

CHAPTER 3: EXPEREMENTAL SETUP AND PROCEDURE ................................. 17

3.1 Sample Preparation ................................................................................................. 17

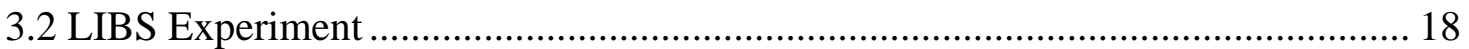


3.2.1 Modular Collimator System......................................................................... 18

3.2.2 Spectrograph Selection .............................................................................. 19

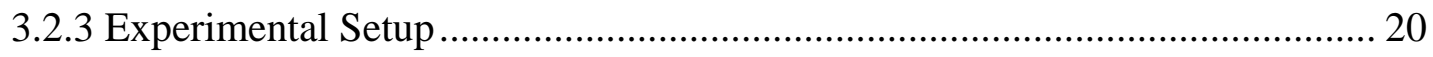

3.3.2 Static LIBS and Dynamic LIBS................................................................ 21

3.3.3 LIBS Testing Procedure........................................................................ 23

3.3.4 File Processing Procedure................................................................................ 25

CHAPTER 4: RESULTS AND DISCUSSION ............................................................ 27

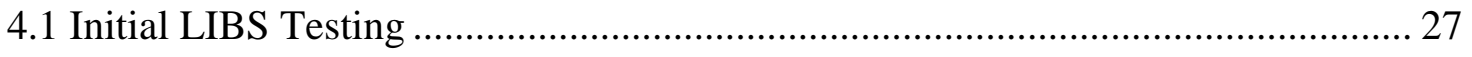

4.2 LIBS Testing of Synthetic Mixtures ................................................................. 28

4.3 Static LIBS Analysis.................................................................................... 29

4.3.1 Qualitative Analysis............................................................................... 29

4.3.2 Quantitative Analysis ............................................................................... 29

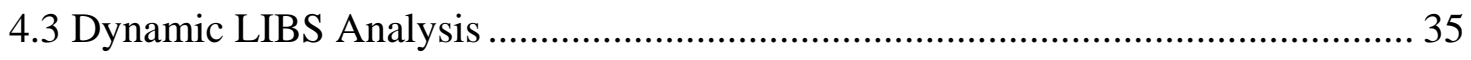

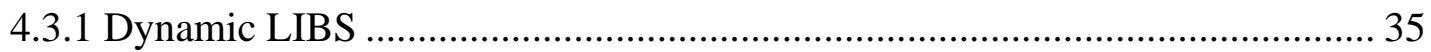

4.3.2 Calculation of the Dynamic LIBS Time Frame ............................................... 36

4.3.3 Signal Processing and Analysis ..................................................................... 39

CHAPTER 5: CONCLUSIONS AND RECOMMENDATIONS................................ 51

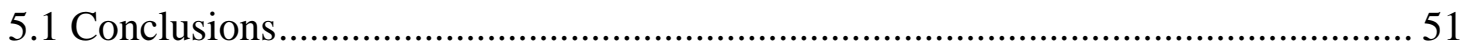

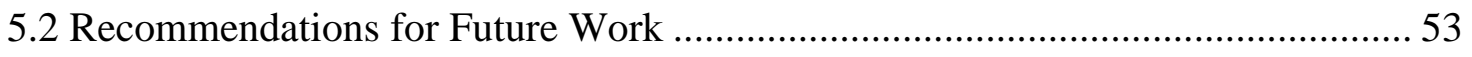

5.2.1 System Improvements.................................................................................. 53

5.2.2 The Ideal LIBS Apparatus ........................................................................... 54

REFERENCES.................................................................................................................. 56

APPENDIX A: FILE PROCESSING ..............................................................................60 
APPENDIX B: FIGURES OF RAW DATA FROM STATIC LIBS TESTING ..... 73

APPENDIX C: PROCESSED DATA FILES FROM STATIC LIBS TESTING..... 83

APPENDIX D: DYNAMIC LIBS DATA SETS...............................................................90 


\section{LIST OF TALBES}

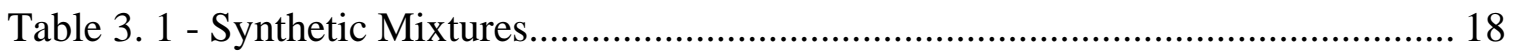

Table 3. 2 - Elemental Concentrations of Synthetic Mixtures....................................... 18

Table 4. 1 - Emission Lines Observed in 431-452nm wavelength range. ........................ 28

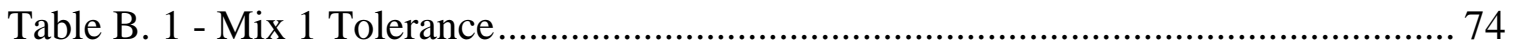

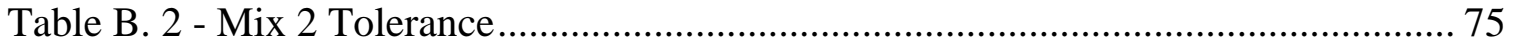

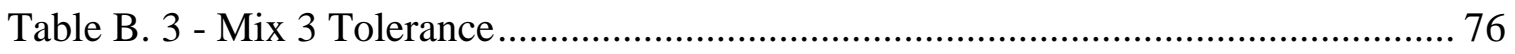

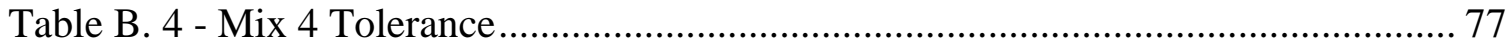

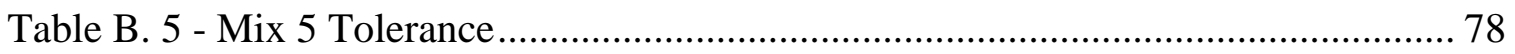

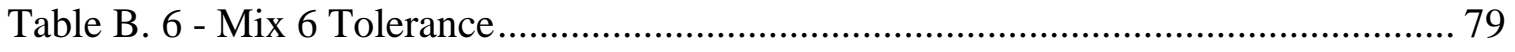

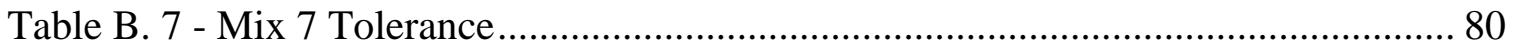

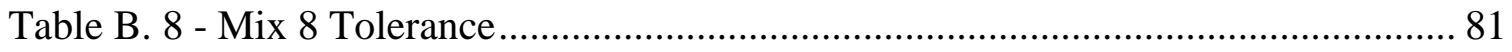

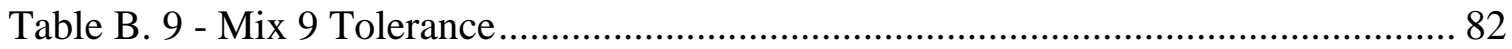

Table D. 1 - Number of Dynamic Shots per Synthetic Mixture ................................... 90 


\section{LIST OF FIGURES}

Figure 3. 1 - Sample Preparation System.................................................................... 17

Figure 3. 2 - LIBS Testing Schematic ........................................................................... 21

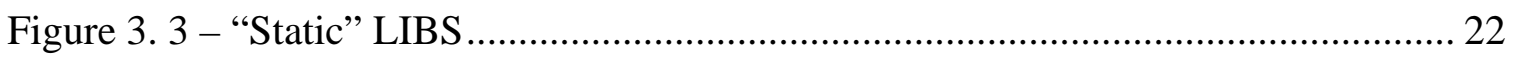

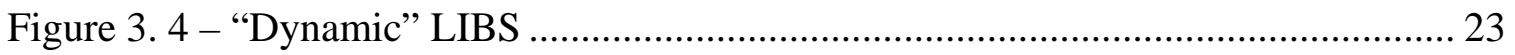

Figure 3.5 - LIBS Testing Apparatus .......................................................................... 25

Figure 4. 1 - the dimensions of the Modular Collimator System...................................... 19

Figure 4. 2 - Static LIBS Raw Data, mix\#1, shot 59.................................................... 29

Figure 4. 3 - 100 Shot Average of Mix 1 for qualitative and quantitative analyses ........ 30

Figure 4. 4 - Correlation of the 445nm Carbon - related line............................................. 31

Figure 4. 5 - Correlation of the $447 \mathrm{~nm}$ Carbon - related line........................................... 31

Figure 4. 6 - Correlation of the 436nm Mercury Emission Line....................................... 33

Figure 4. 7 - Correlation of the 432nm Iron Emission Line............................................... 33

Figure 4. 8 - Correlation of the 439nm Iron Emission Line............................................ 34

Figure 4. 9 - Correlation of the 441nm Iron Emission Line............................................. 34

Figure 4. 10- Images and Intensity Profiles of Static and Dynamic LIBS ........................ 36

Figure 4. 11 - Modeling in OSLO. The spark image was scanned to form a 2mm line on

the entrance slit to the spectrograph............................................................................. 38

Figure 4. 12 - Original and Background Corrected Signals.............................................. 39

Figure 4. 13 - Traveling beam incident on the lens ......................................................... 40

Figure 4. 14 - Eclipsing Function ..................................................................................... 41

Figure 4. 15 - Convoluted Emission Line Data separated by 10 counts for clarity ........... 42 
Figure 4. 16 - Emission Lines with eclipsing correction at later spark life separated by 20 counts for clarity 43

Figure 4. 17 - Background Radiation Decay ........................................................... 43

Figure 4. 18 - C 445nm temporal behavior............................................................ 44

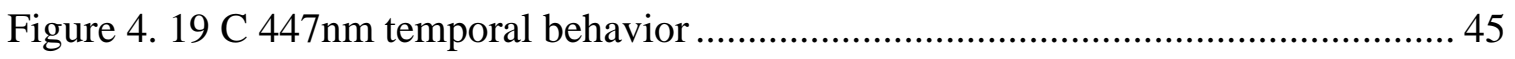

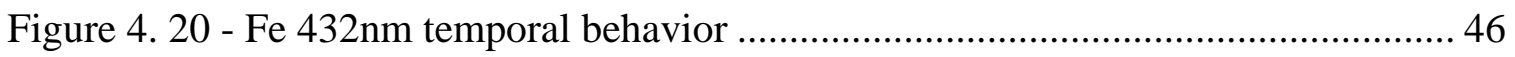

Figure 4. 21 - Fe 439nm temporal behavior ......................................................... 47

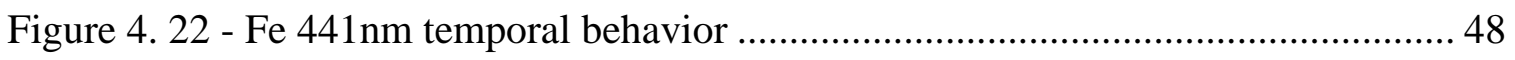

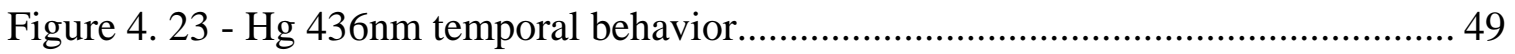

Figure 4. 24 - Comparison of Iron and Mercury Lines in Mix9 .................................. 50

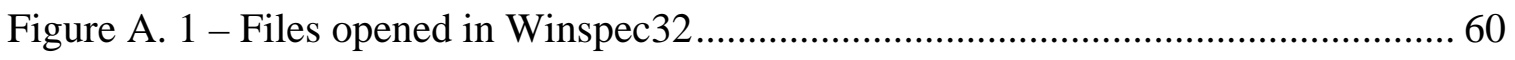

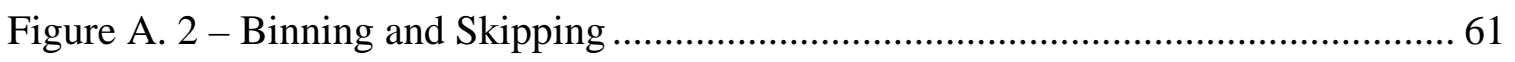

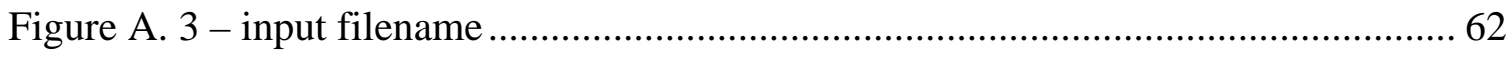

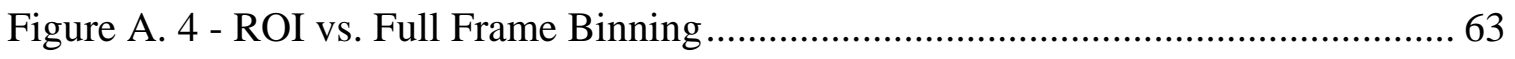

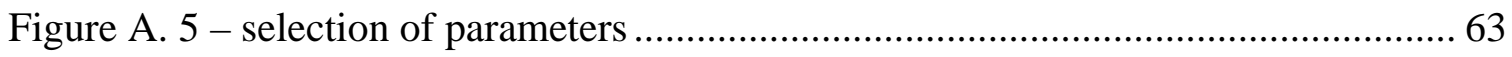

Figure A. 6 - selection of output filename ......................................................... 64

Figure A. 7 - a binned file in Winspec32 …......................................................... 65

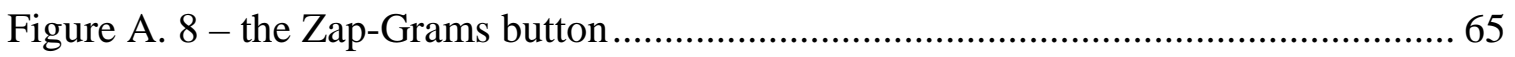

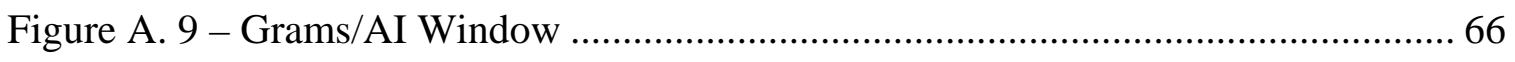

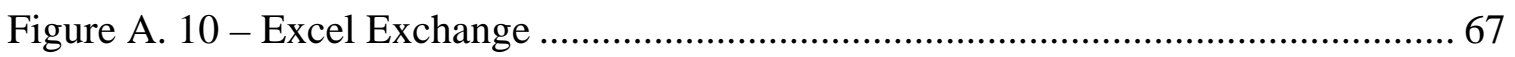

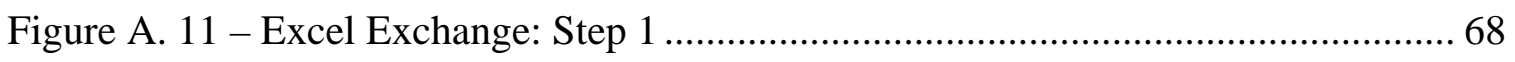

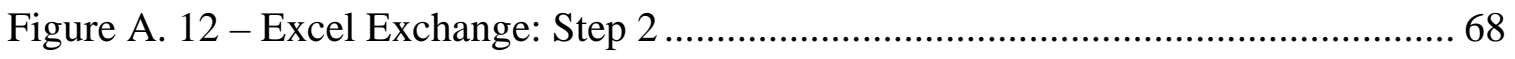

Figure A. 13 - Excel Exchange: Step 3 .............................................................. 68 


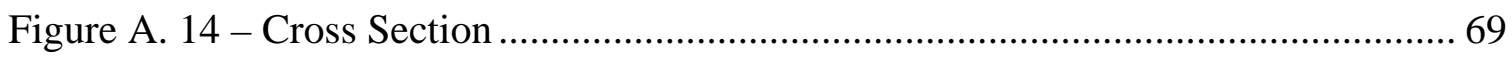

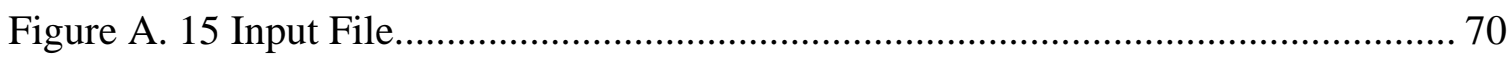

Figure A. 16 - Parameters of Cross Sectioning.............................................................. 71

Figure A. 17 - Output File......................................................................................... 72

Figure A. 18 - mix9shot11C447 final product......................................................... 72

Figure B. 1 - First 10 shots of Synthetic Mix 1........................................................... 74

Figure B. 2 - First 10 shots of Synthetic Mix 2. ............................................................ 75

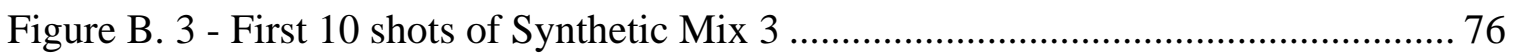

Figure B. 4 - First 10 shots of Synthetic Mix 4 ............................................................. 77

Figure B. 5 - First 10 shots of Synthetic Mix 5 .............................................................. 78

Figure B. 6 - First 10 shots of Synthetic Mix 6 ........................................................... 79

Figure B. 7 - First 10 shots of Synthetic Mix 7 .......................................................... 80

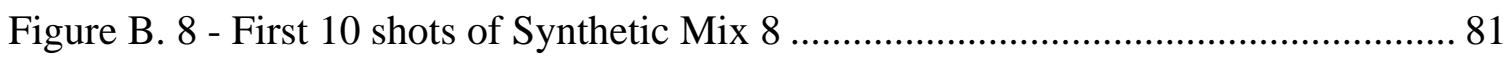

Figure B. 9 - First 10 shots of Synthetic Mix 9 ............................................................. 82

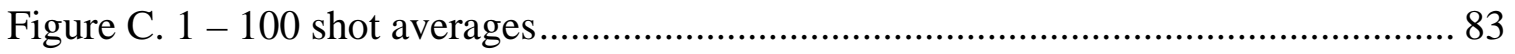

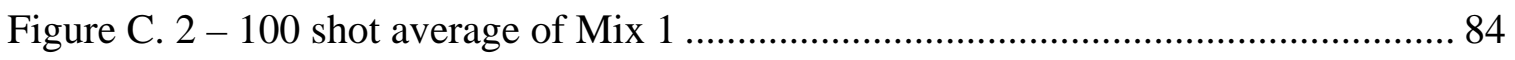

Figure C. 3 - 100 shot average of Mix 2 …………………......................................... 84

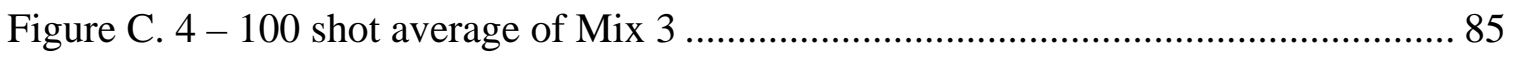

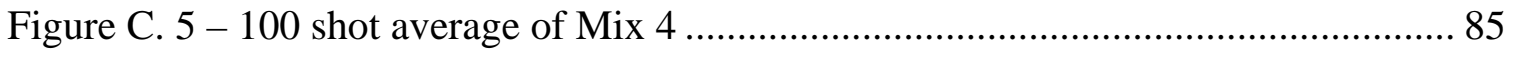

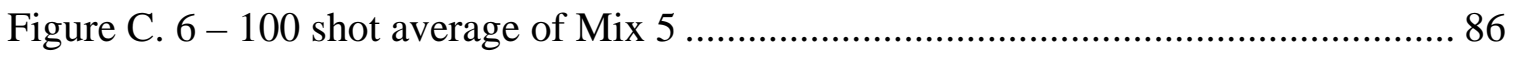

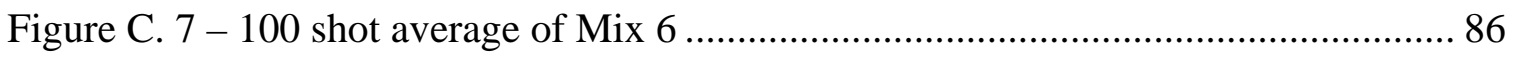

Figure C. 8 - 100 shot average of Mix 7 ....................................................................... 87 


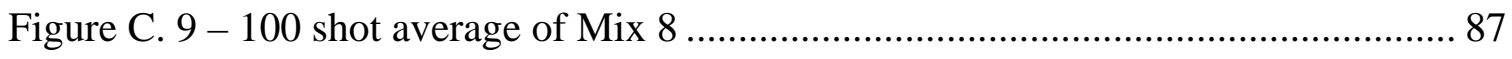

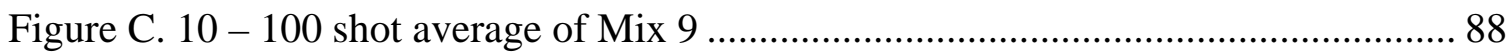

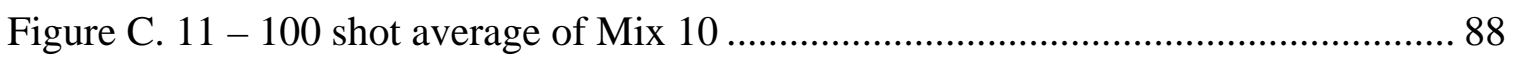

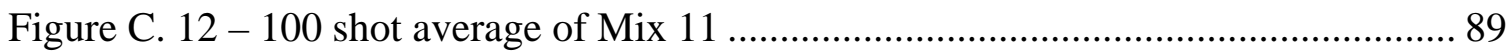

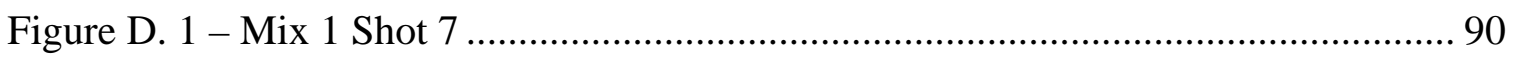

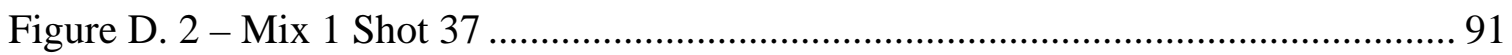

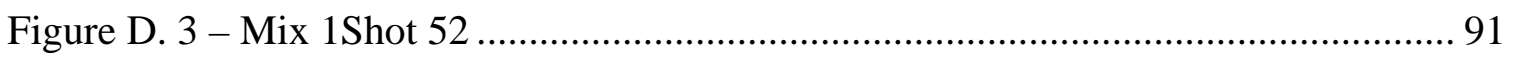

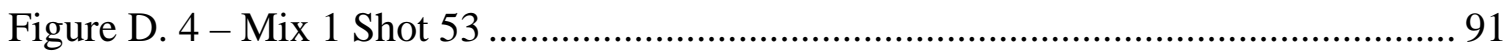

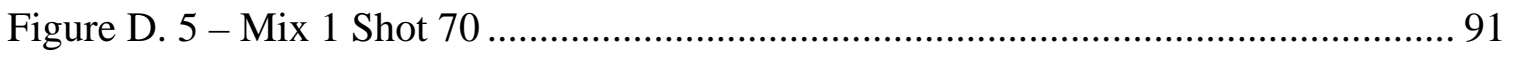

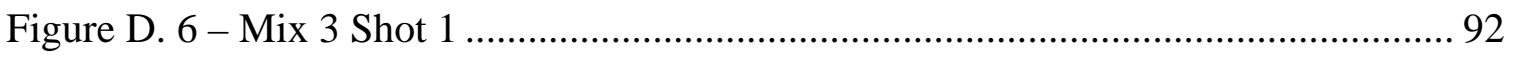

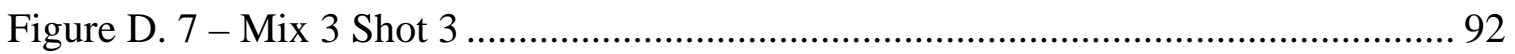

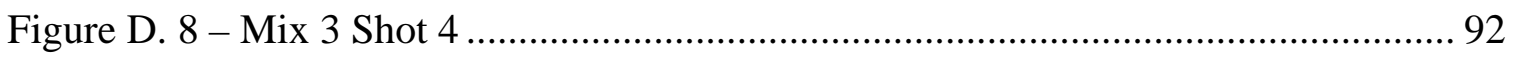

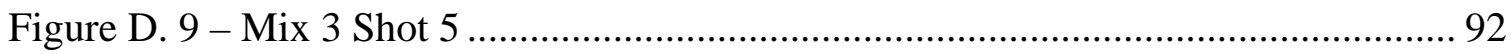

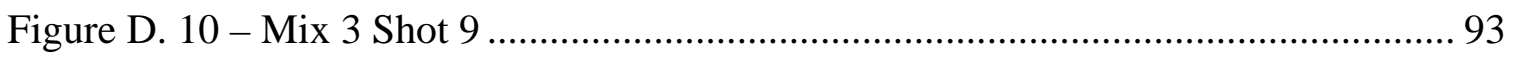

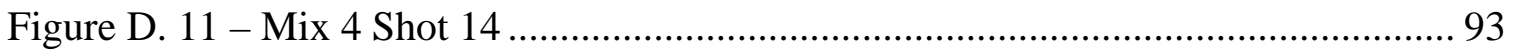

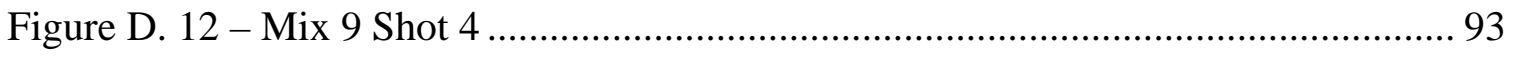

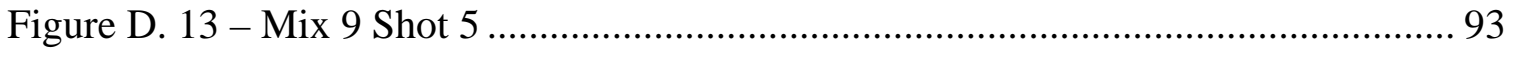

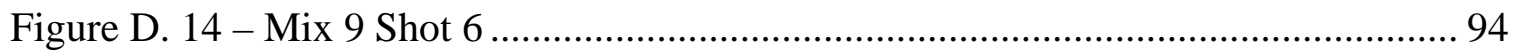

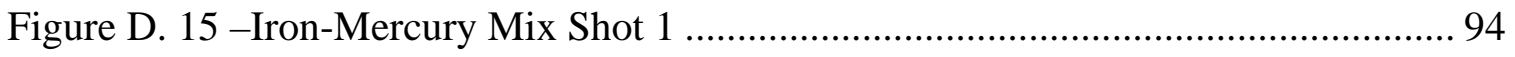

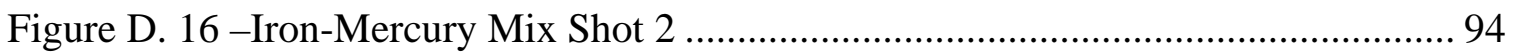

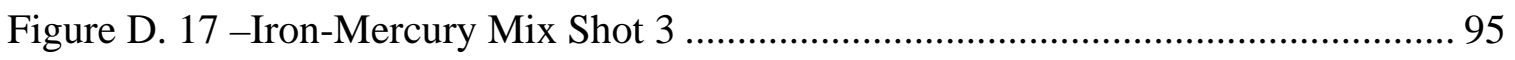

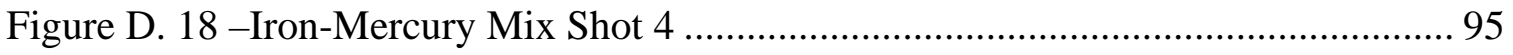

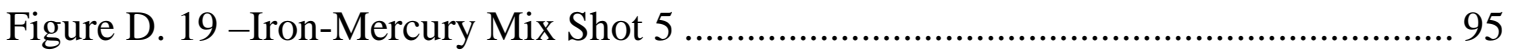




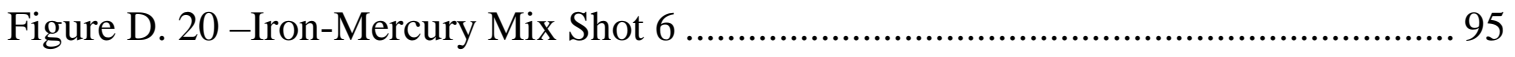

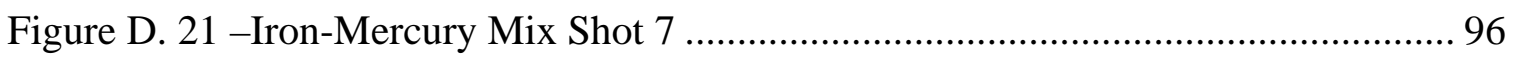

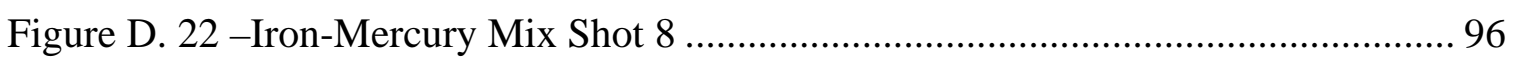

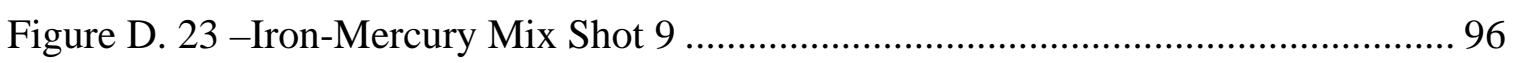

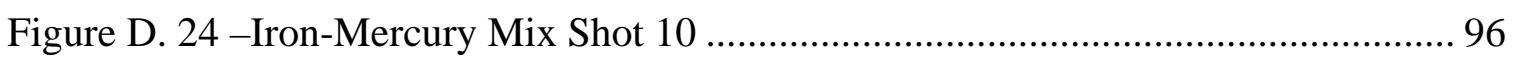




\section{CHAPTER 1: INTRODUCTION}

\subsection{LIBS fundamentals}

Laser Induced Breakdown Spectroscopy (LIBS) is a technique that uses a high peak power laser to disintegrate a sample of a desired material into excited atoms and ions resulting in plasma formation. During the early stages of the plasma development (less than 10 microseconds), the plasma temperature goes up to 10,000 to $25,000 \mathrm{~K}$ [1]. At this temperature, the plasma has sufficient energy to break molecular bonds to form constituent atoms and excite electrons of neutral atoms and ions into excited electronic states. All chemical bonds are broken resulting in highly excited, and unstable, atoms, ions and free electrons. The free electrons are absorbed by the atoms and ions in the plasma, resulting in a continuum emission. As the plasma cools, characteristic photons are emitted as the excited ions and atoms stabilize, resulting in the formation the ionic and atomic emission lines of the elements, i.e. a spectrum. Each element has a unique spectrum of atomic and ionic emission lines. The emitted light from the laser-induced

plasma can be collected and used to provide information on the elemental composition of the material by means of spectroscopy.

There are several key advantages to LIBS that can be beneficial for an on-line monitoring and diagnostic. LIBS is a non-contact, minimally destructive test. It requires the light collected from the plasma created by the laser and destroys an insignificant amount of particles (i.e. in the tens of nanograms). Because LIBS is an optical technique, it can be used for deployment in hostile environments. After sufficient calibration, LIBS requires no sample preparation, which makes it a candidate for development of a portable system, easily deployed for field use. 
Since 1990, several LIBS systems have been developed in different international laboratories with different analytical applications. Common tasks, such as certification of metal contents in alloys, and detection of trace metals in soils for environmental protection, have already employed the use of LIBS instruments [2].

In conducting experiments with the LIBS technique, basic principles of operation and a fundamental understanding of spectroscopy must be established. After establishing fundamentals, results of experiments will be gathered to support the idea of using LIBS as a method for determining the elemental analysis of coal fines.

The fundamental understanding of the LIBS process comes from Max Planck, who proposed the quantum theory in order to explain the properties of radiation emitted by heated bodies. This theory was later extended to include the emission and absorption processes. There are two important postulates of the quantum theory [3]. The first postulate states that atoms, ions and molecules can exist only in certain discrete states, characterized by definite amounts of energy. During plasma formation, all bonds are broken, resulting in emission of free electrons, creating a continuum emission. The continuum emission disappears quickly as the free electrons are captured by atoms and ions. When a specie changes its states, it absorbs or emits an amount of energy exactly equal to the energy difference between the states. According to the second postulate, when atoms, ions or molecules absorb or emit radiation in making the transition from one energy state to a second, the wavelength, $\lambda$ of the radiation is related to the energy difference between the states by the following equation.

$$
\Delta \mathrm{E}=h c / \lambda \quad \ldots(1-1)
$$


The quantity $\Delta \mathrm{E}$ is the difference between the higher and lower energy states, $c$ is the speed of light ( $3 \times 10^{8}$ meters/second) and $h$ is Planck's constant $\left(6.626 \times 10^{-34} \mathrm{~J} / \mathrm{s}\right)$. As energy changes from one state to the other within the laser-induced plasma, it is accompanied by the emission of light. This light is then collected and analyzed using the concepts of the science of optical spectroscopy.

Spectroscopy is the science that deals with an interaction between various types of electromagnetic radiation and matter [3]. The specific type of spectroscopy that is utilized here is optical atomic spectroscopy. The spectra observed are the atomic emission spectra, which is a result of Planck's quantum theory, briefly explained above. Once the energy has been released, the light must be collected and treated accordingly to obtain the spectrum for further analysis. This involves the use of optics to collect the emitted light and direct it to a spectrograph, which disperses the light and re-focuses it as a spectrum on to a detector for data collection.

The conventional tools for experiments involving LIBS include a high powered, pulsed laser to atomize the substance, optical elements for collection and directing of light emitted from the laser-induced plasma, a spectrograph for separating the light by wavelength, and a detector, often a CCD (charge-coupled device) to collect the image produced by the spectrograph. A high powered laser is used to sample the specimen, which emits light into $4 \pi$ steradians. A fraction of this light is collected by a lens and directed into the entrance slit of the spectrograph with the use of lenses and mirrors. The spectrograph, with a combination of gratings and mirrors, separates the light in accordance to its wavelength. After the light goes through the spectrograph, it lands on the pixels of a CCD, or intensified CCD (ICCD). The CCD then collects the data, and 
relays it to the CCD controller. The CCD controller processes the data and sends it to a computer, which displays the data as a graph of intensity with respect to wavelength on the computer monitor. Although the overall process is dependent upon many variables, such as timing and the processing speed of the host computer, LIBS data is capable of being produced in almost real time, displaying spectroscopic data in less than a second.

With minimal sample preparation, and data collection at almost real time, LIBS would seem like an ideal application in many fields including soil testing, on-line and environmental monitoring. LIBS is being advanced in laboratories throughout the world, and some companies are now selling commercial packages [4]. However, real-world applications present technical difficulties including analytical issues such as calibration, precision, accuracy, and interferences. Instrumental ruggedness is also an issue [4]. The benefit to cost ratios for LIBS experiments will constantly change according to each application.

\subsection{Research Objective}

The objective of this project was to develop a LIBS system to determine qualitatively as well as quantitatively the content of carbon, sulfur, iron and mercury in coal and test the limits of detection of the elements of interest in standard atmospheric conditions. This research was to consist of a fundamental study of laser-induced breakdown spectroscopy (LIBS) applied to coal samples, coal fines, and fly ash. This study is to include the use of a unique capability of temporal resolution by means of a polygonal scanning mirror. Characteristic elemental emissions are to be observed and a method established for the quantification of the elements of interest. An experimental 
method for observing temporal characteristics of the emission lines of interest was also developed. 


\section{CHAPTER 2: LITERATURE REVIEW}

\subsection{Coal Constituents and Analyses}

This section establishes a background of coal with specific interest in constituents and reviews current ASTM standards for the analysis of elements such as carbon, iron (trace metals), sulfur and mercury, as well as an ASTM method similar to LIBS.

\subsubsection{Coal Rank}

Coal ranges from a soft, moist brownish material to a hard, black solid. Coal varies in physical appearance, material properties, and elemental composition. Because of the wide variation in coal, it is necessary to have a system that characterizes coal. Traditionally, there were three kinds of analyses that characterize coal; proximate analysis, which determines moisture, fixed carbon, volatile matter, and ash; heating value, the analysis that determines the amount of energy that can be obtained by burning coal; and ultimate analysis, an elemental analysis that normally determines carbon, hydrogen, oxygen, sulfur, nitrogen, and ash [5]. Each analysis has standard procedures set by the American Society for Testing and Materials (ASTM).

These characteristics of coal vary widely from seam to seam. To classify coal by these characteristics, ASTM developed the classification of coal by ranks. These ranks of coal provide the physical description, material properties and elemental composition associated with those particular ranks of coal. Brown coal and lignite have a brownish color; have the least amounts of carbon, and the highest moisture content of the ranks. Bituminous coal is the predominant coal that is used in the United States. Moisture and volatile matter are lower and heating value is higher than that of the lignite and 
subbituminous coals (coals ranked between bituminous coal and lignite). With low moisture and volatile matter content, high heating values, and a clean burning flame, anthracite ranks highest among coals.

\subsubsection{Coal Analyses}

With coal ranks having been established, the determination of coal's constituents can be estimated by the particular rank of the specific coal. Of particular interest within the scope of this project is the elemental analysis of bituminous coal, particularly coal mined from the Appalachian Mountain Range. The elements of interest are carbon, iron, mercury and sulfur. Carbon and sulfur are determined in the ultimate analysis of coal, for which there is an ASTM standard procedure. Mercury has 2 different ASTM standard procedures for analysis, and iron can be found in ash analysis as well as analysis of trace elements in coal. These tests are fully described in the Annual Book of ASTM Standards.

Before the analysis, most of the test methods require that coal samples must be prepared using ASTM method D2013. In this method, a gross sample of coal is divided and crushed to pass a number of sieves, finally ending in a 60 sieve, which is approximately 250 microns. The procedure also requires that the final sample must weigh no less than 50 grams [6]. After procedure D2013, the sample can be analyzed by other ASTM standard procedures. Example standard test methods for the elements of interest are mentioned below.

ASTM method D 3178 -89 (2002) states that carbon and hydrogen analyses are

determined by burning a weighed quantity of sample in a closed system and fixing the 
products of combustion in an absorption train after complete oxidation and purification from interfering substances [7].

According to ASTM method D 3177 -02, sulfur can be analyzed in two different methods, the Eschka method, and the Bomb Washing method. The analysis of sulfur done by the Eschka method takes a weighed sample of coal and Eschka mixture are mixed and ignited together (Eschka's mixture is magnesium oxide and calcium carbonate). The sulfur is dissolved in hot water and then precipitated from the resulting solution as barium sulfate. In the second method, sulfur is precipitated as barium sulfate from oxygen bomb calorimeter washings. In each method, the precipitate is filtered, ashed and weighed [8].

Ash is determined by the weight of the residue of burned coal or coke under rigidly controlled parameters of weight, temperature, time, atmosphere and equipment conditions [9]. Ash composition differs from the inorganic coal composition, as chemical reactions have taken place during the burning period. Because incineration conditions differ from furnace to furnace, ash composition will be varied.

Mercury, because of its environmental concern, is of particular importance to observe. A current standard of mercury determination in coal is by direct combustion analysis, where controlled heating of the sample in oxygen liberates mercury. The sample is dried out by heating, then thermally and chemically decomposed. The decomposition products are carried by flowing oxygen and passed through a catalytic section and then through an algamator, where mercury is selectively trapped. The algamator is rapidly heated to release the mercury vapor, which is carried by flowing 
oxygen through absorbance cells positioned in the light path of single wavelength atomic absorption spectrophotometer, which is adjusted to the $253.7 \mathrm{~nm}$ wavelength [10].

ASTM standard D6349-01 [11] relies on atomic emission spectroscopy by means of an inductively coupled plasma (ICP). ICP is similar to LIBS because it relies on emission spectroscopy. In this method, the sample is ashed and dissolved in a mild acidic solution. The solution is nebulized and transported to the produced plasma torch, where excitation and emission occur. As within LIBS, a grating monochromator system is used to separate the emission lines. A photodiode array detector monitors intensities of the lines.

\subsubsection{Coal Constituents}

When coal is carefully analyzed for major, minor, and trace constituents, almost every element will be found, however, the main constituent of coal is carbon. Carbon content in coal is what classifies it by rank. The final maturation of coal is a form of pure carbon [5]. Anthracite, which is the "highest" ranked coal, has carbon concentrations from 86 to 98 percent. Other coals contain anywhere between 69 and 86 percent carbon. Proximate analyses and Ultimate analyses show that oxygen content in coal will decrease as carbon content increases. Oxygen in coal will range from 25\% to almost nothing. Hydrogen and nitrogen show little change being around 5.5\% and 1\%, respectively, while sulfur ranges from 0.5 to 8 percent in the United States coal beds [5]. Other elements found in coal are sodium, calcium, magnesium, potassium, aluminum, silicon, iron and mercury.

If it is necessary to have the concentrations of elements known in coal prior to testing, or if the coal will be used as an internal standard for a test method, a sample of 
analyzed coal can be purchased by the National Institute of Standards and Technology (NIST). NIST sells several samples of analyzed coal. The current samples of coal provided by NIST are bituminous and analyzed for sulfur and mercury content.

\subsection{Experiments of LIBS applied to coal}

Because coal is a heterogeneous substance, the application of LIBS to analyze the coal can be difficult. Only a few articles have been published that discuss the application of LIBS to coal. Most LIBS experiments applied to coal have been applied to the coal power industry as an on line monitor. The collection and processing of the LIBS spectrum is done from an experimental approach. LIBS signals are quantified by the intensities of the elemental emissions in question. The following experiments described below present methods for analysis of the coal spectrum.

LIBS was applied to Australian lignite at the Cooperative Research Centre for Clean Power from Lignite. Body and Chadwick developed and applied the LIBS method to the coal power industry [12].

The design of their collection system incorporated a parallel processing design, utilizing multiple spectrographs, CCD detectors, and acquisition electronics. The configuration used a $90 \mathrm{~mJ}$ pulsed Nd:YAG laser, $0.2 \mathrm{~m}$ optical spectrographs with a resolution of 3 angstroms, and gated CCD detectors. The four spectrographs were typically used to cover the wavelength range $180-800 \mathrm{~nm}$. The apparatus was fully software controlled. Samples presented for the analyses were on a fast translation stages, which reduced the matrix effects induced by laser-sample interaction.

Acquisition of the data involved moving the specimen, firing the laser, and storing the resulting spectra from each CCD. To avoid the broadband emission from the plasma, 
the acquisition was delayed for approximately one microsecond. During acquisition, the instruments were programmed to analyze 250 laser pulses, which results in a total of 1000 spectra for analysis.

Calibration was performed by preparing a lignite standard with high concentrations of silicon, aluminum, iron, calcium, sodium, and magnesium. This was done by crushing and blending four samples from different mine areas. The sample was blended and tested until the uniformity of the sample was confirmed. The process was repeated until five well characterized samples of low and high ash lignite samples were developed. Samples taken and analyzed by LIBS were analyzed by two alternative test methods. In addition to the standards, 90 other samples were tested. The analysis procedure of the experiment was to prepare subsequent unknowns in the same way that the calibration standards. From these tests, detection limits were obtained as low as 3 parts per million in sodium.

The application of LIBS has also been applied to detect unburned carbon in fly ash in Japan by Kurihara et al [13]. An automated LIBS unit was developed and implemented within a 1000-MW coal-fired power plant. Kurihara et al reported that quantitative measurements from LIBS and the conventional standard (Japanese Industrial Standard 8815) were in agreement of each other.

In Kurihara's research, the method of LIBS measurements relied on computation and calibration standards, as calibration of the LIBS signal was necessary for quantitative analysis. Kurihara used system of equations was used to describe the emission formed from the plasma. The equation for the intensity of an atomized species was stated, based on the assumption of a uniform plasma temperature. 


$$
I_{i}=A_{i} n_{i} \sum_{j}\left\{g_{i}^{(j)} \exp \left[-E_{i}^{(j)} / \mathbf{k T}\right]\right\}
$$

Where $I_{i}[J / s]$ is the emission intensity of species $i, A_{i}\left[\mathrm{~J} \mathrm{~m}^{3} / \mathrm{s}\right]$ is a constant that refers to the energy transition of species $i, n_{i}\left[1 / \mathrm{m}^{3}\right]$ reflects the concentration of species $i, g_{i}^{(j)}$ is the statistical weight of species $i$ at upper energy level $j, E_{i}^{(j)}[J]$ is the upper level energy of species $\mathrm{i}, \mathrm{k}$ is the Boltzmann constant, and $\mathrm{T}[\mathrm{K}]$ is the plasma temperature.

Temperature was then determined by measuring two different atomic spectral lines of the same atom and determining the ratio between them. If the statistical weights, $\mathrm{g}_{\mathrm{i}}^{(1)}$ and $\mathrm{g}_{\mathrm{i}}^{(2)}$ are known, then the plasma temperature can be directly calculated using the ratio of the two.

$$
\frac{\mathrm{I}_{\mathrm{i}}^{(1)}}{\mathrm{I}_{\mathrm{i}}^{(2)}}=\frac{\mathrm{g}_{\mathrm{i}}^{(1)}}{\mathrm{g}_{\mathrm{i}}^{(2)}} \exp \left[-\frac{\mathrm{E}_{\mathrm{i}}^{(1)}-\mathrm{E}_{\mathrm{i}}^{(2)}}{\mathrm{kT}}\right]
$$

From this second equation, $\alpha_{\mathrm{i}}$, which is a variable factor related to species $\mathrm{i}$, which contains the plasma's temperature and pressure correction factors was derived by Kurihara et al. The variable is defined by use of the intensity ratio in equation (2-2).

$$
\alpha_{\mathrm{i}}=\mathrm{K}_{\mathrm{i}} \mathrm{I}_{\mathrm{i}}^{\mathrm{b0}}\left[\mathrm{I}_{\mathrm{i}}^{(1)} / \mathrm{I}_{\mathrm{i}}^{(2)}\right]^{\mathrm{b} 1}
$$

$K_{i}$ and $b_{0}$ are correction factors for species $I_{i}$. $I_{j}{ }^{(1)}$ and $I_{j}{ }^{(2)}$ are emission intensities from upper energy levels 1 and 2 of species $\mathrm{j}, \mathrm{b}_{\mathrm{i}}$ is the plasma temperature correction factor for the emission pair $\mathrm{I}_{\mathrm{j}}^{(1)}$ and $\mathrm{I}_{\mathrm{j}}^{(2)}$. The correction factors were determined under measurement conditions that contained parameters such as laser intensity, pressure and gas composition.

Since typical analytical constituents of fly ash are $\mathrm{SiO}_{2}, \mathrm{Al}_{2} \mathrm{O}_{3}, \mathrm{Fe}_{2} \mathrm{O}_{3}, \mathrm{CaO}$ and unburned carbon, the carbon content in the fly ash was calculated from the emission 
intensities of Si, $\mathrm{Al}, \mathrm{Fe}, \mathrm{Ca}$ and $\mathrm{C}$. The unburned carbon in the fly ash was stated as the following:

$$
\% \mathrm{C}=\frac{\alpha_{\mathrm{C}} \mathrm{L}_{\mathrm{C}} / \mathrm{I}_{\mathrm{Si}}}{1+\alpha_{\mathrm{C}} \mathrm{L}_{\mathrm{C}} / \mathrm{I}_{\mathrm{Si}}+\alpha_{\mathrm{Al}} \mathrm{I}_{\mathrm{Al}} / \mathrm{I}_{\mathrm{Si}}+\alpha_{\mathrm{Fe}} \mathrm{I}_{\mathrm{Fe}} / \mathrm{I}_{\mathrm{Si}}+\alpha_{\mathrm{Ca}} \mathrm{I}_{\mathrm{Ca}} / \mathrm{I}_{\mathrm{Si}}}
$$

The main constituents of the fly ash were detected in the wavelength range from 240 to $340 \mathrm{~nm}$. The analysis of the $\mathrm{C} / \mathrm{Si}$ and $\mathrm{Fe} / \mathrm{Si}$ ratios used a high resolution spectrograph, where the $\mathrm{Al} / \mathrm{Si}$ and $\mathrm{Ca} / \mathrm{Si}$ ratios were found with a wide-range spectrograph. Similarly, the concentrations of Si, Ca, Al and Fe in the fly ash were determined from the signal intensity for each emission wavelength by equation (2-4). As stated before, the measurements from the LIBS testing done by Kurihara were in agreement with the industrial standard stated earlier (Japanese Industrial Standard 8815).

LIBS was applied to coal particles as early as 1990 at the Combustion Research Facility at Sandia National Laboratories by Ottesen, et al [14], when performing studies on single particles for real-time, in situ monitoring. The results have shown that laser spark emission spectroscopy (synonymous with LIBS) proved to be a useful technique for determining the elemental composition of single coal particles in flowing environments. In this flowing environment, $\mathrm{Li}, \mathrm{Na}, \mathrm{K}, \mathrm{Mg}, \mathrm{Ca}, \mathrm{Ba}, \mathrm{Sr}, \mathrm{Al}, \mathrm{Si}, \mathrm{Ti}, \mathrm{Mn}$, and $\mathrm{Fe}$ in addition to $\mathrm{C}, \mathrm{H}, \mathrm{O}$ and $\mathrm{N}$ were detected. In this experiment, sulfur could not be observed. A major concern of the experiment was the inability to resolve elements with interfering wavelengths. The use of a half meter spectrograph with a 300 grove $/ \mathrm{mm}$ grating provided a wide spectral range of $170 \mathrm{~nm}$, however, the resolution was sacrificed for range. The relative concentrations of the elements on an atomic bases were determined by using ratio of corrected, integrated intensities for several emission lines 
along with an effective plasma temperature in the Boltzmann or Saha relations [14]. The detection limits recorded for this experiment was $100 \mathrm{ppm}$.

Wiesburg et. al. performed a recent experiment of LIBS applied to coal at Energy Research Company (ERCo). From their experiments, they obtained values of hydrogen, carbon, metals, ash content, and nitrogen [15]. Eleven samples from different coals were taken and pressed. The coal samples were then subject to LIBS testing from an inert gas environment at a pressure at $50 \mathrm{kpa}$, one half of atmospheric pressure.

To collect the emitted light from the laser spark, viewing lenses focused light onto the ends of two fiber optic cables, one was to a conventional Czerny-Turner scanning spectrograph system, while the other was connected to a broadband Echelle spectrograph, capable at viewing wavelengths from $200-780 \mathrm{~nm}$. Elements such as hydrogen, oxygen and nitrogen were found in the far visible to near infrared region, while sulfur was found separately at $857 \mathrm{~nm}$. Other elements determined in the study were $\mathrm{Ca}, \mathrm{Al}, \mathrm{Fe}, \mathrm{K}, \mathrm{Mg}, \mathrm{Na}$, $\mathrm{Si}$, Ti, and Cr. Two elements, arsenic and mercury were also measure with LIBS testing, although the coal samples were doped to approachable detection limits, both of which where reported at 36ppm [15]. Mercury's emission line at $254 \mathrm{~nm}$ was observed in this experiment, however, there was interference with iron reported.

\subsection{Experiments of LIBS on Elements of Interest}

This section reviews the elements of interest in regards to sensitive wavelengths, interfering wavelengths, detection limits, and experimental conditions. 


\subsubsection{Carbon}

LIBS has been used to determine carbon in soils, coal, and gas/air mixtures containing $\mathrm{CO}$ and $\mathrm{CO}_{2}$. The most common line observed in carbon lies in the UV range at 247.9nm. This emission line has been observed in experiments on coal, and in soils. Martin et. al. reported that a plot of intensity with background correction plotted as a function of concentration showed a linear relationship when determining total carbon in soils [16]. Also, because of the experimental apparatus, an iron emission line at 248.4nm was said to have interfered with the carbon signal. Sturm and Noll conducted a LIBS experiment of air/gas mixtures for multi-element measurements of carbon, hydrogen, oxygen and nitrogen [17]. Using ratios of carbon to oxygen, Sturm and Noll developed excellent calibration curves correlating atomic abundance ratio to the ratio of the 2 peak areas. While the $247.9 \mathrm{~nm}$ emission line is commonly used as a good emission line to observe, Ferioli, Puzinauskas, and Buckley utilized the 711.3nm emission line to observe carbon in their on-line engine equivalence ratio measurements [18]. Carbon has been detected at a concentration of less than $10 \mathrm{ppm}$ in the ultraviolet range at 193.1nm [19] as well as in the infrared at $833.52 \mathrm{~nm}[20]$.

\subsubsection{Iron}

According to Wiesburg [15], iron interferes with the $253.7 \mathrm{~nm}$ mercury line, and according to Martin [16], it also interferes with the $248.4 \mathrm{~nm}$ carbon emission line. The MIT wavelength tables have so far recorded 4757 emission lines between 200 and 1000 nm for iron [21]. The detection limits can be as low as 0.01 parts per million [20] in the ultraviolet range. Iron lines appear all over the ultraviolet, visible, and infrared spectrum. 


\subsubsection{Mercury}

Mercury’s strongest observable emission line appears at $253.7 \mathrm{~nm}$ [21]. Because of its strong signal, this line is the most observed in the mercury spectrum. Using LIBS, Lazzari, et. al. [23] reported the sensitivity of this line down to 5 parts per billion in an isolated air atmosphere. Gleason and Hahn reported that the 253.7nm line is selectively quenched by oxygen species during the recombination of atoms in the plasma decay process [24]. In a study conducted by Chen, the $253.7 \mathrm{~nm}$ line could not be observed at all when trying to make in-situ measurements in aerosol of $\mathrm{Hg}$ and $\mathrm{Cr}$ in real time [25]. Instead, the $435.8 \mathrm{~nm}$ line was substituted and observed. Mercury has other sensitive lines at 546.1nm, 404.6nm, 366.3nm, 365.5nm, and 365.0nm [21].

\subsubsection{Sulfur}

Sulfur has 451 emission lines within the range of 200nm to 1000nm [21]. All of these lines have been observed by glow discharge source instead of a spark or ark source. Within the 200 - 1000nm range, the most sensitive lines can be observed at $923.7 \mathrm{~nm}$, 922.8nm, 921.3nm, 469.6nm, 469.5nm, and $469.4 \mathrm{~nm}$, with the $469 \mathrm{~nm}$ series having weaker intensities. LIBS experiments have detected sulfur in an inert gas environment around 857nm [15], while LIBS testing in a vacuum environment reported detection limits at 10 ppm while observing the 180.7nm line [19]. Like mercury, sulfur has been known to have emission lines quenched in air [20]. 


\section{CHAPTER 3: EXPEREMENTAL SETUP AND PROCEDURE}

\subsection{Sample Preparation}

The sample preparation equipment consisted of a scale for massing chemicals, a grinding mill for mixing, and a pellet press and die for making pellets out of the synthetic mixtures. Chemicals used in making the mixtures were all in solid powder form and included graphite, coal (NIST sample 1635), iron, and mercuric oxide. Powders were used because they could be thoroughly mixed by a grinding mill and pressed into solids.

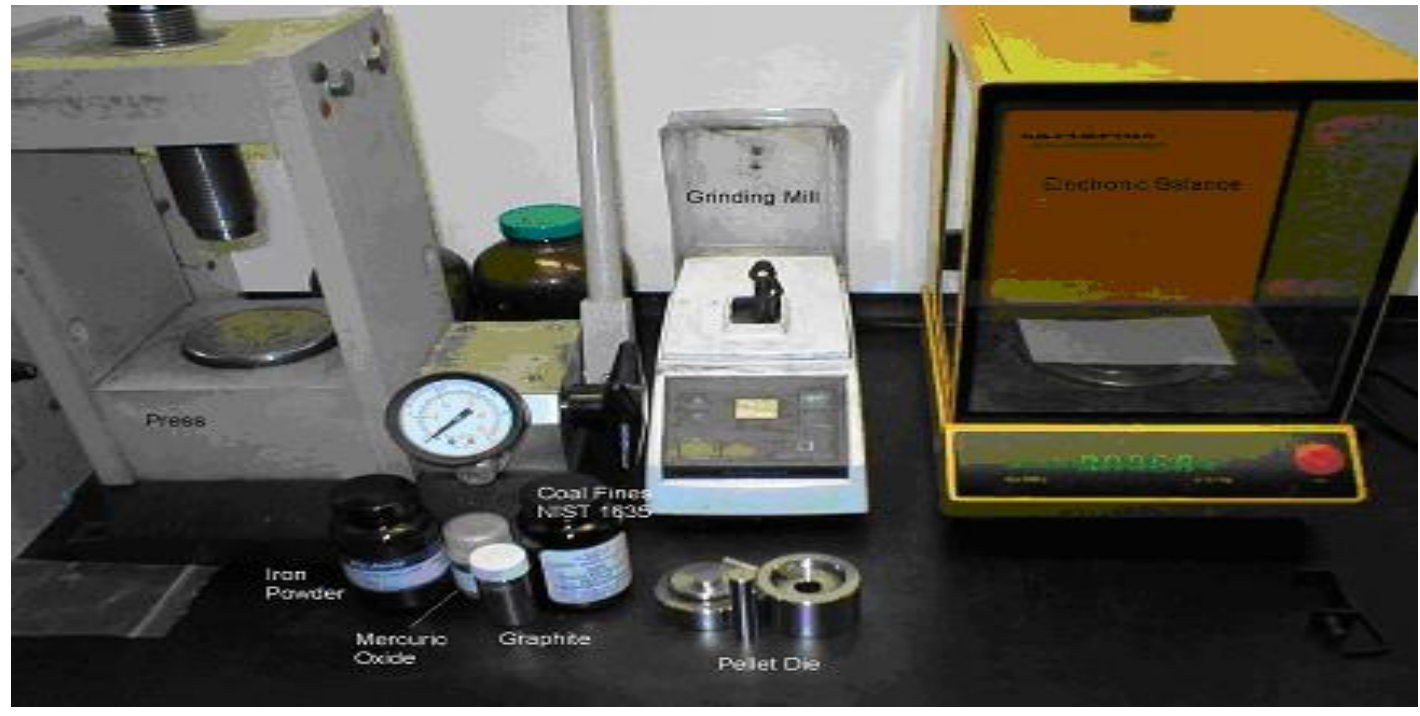

Figure 3. 1 - Sample Preparation System

Eleven synthetic samples were made to test the LIBS process. Each sample contained a mix of graphite, NIST 1635 (sub bituminous coal), iron, and mercuric oxide for elevating mercury levels in the mixtures. The each mixture contained varying concentrations of carbon, iron, and mercury.

Each chemical was initially massed on a tarred weighing dish, and transferred into a labeled grinding capsule. The weighing dish was massed again. The mass recorded 
was the difference between the initial and final masses. The grinding capsule containing the mixture was then placed in the grinding mill (Wig-L-Bug), and ground for one 20 second grinding cycle. The components of the synthetic samples were assumed to be distributed throughout the mixture due to the use of a ball-grinding mill. The synthetic mixture was then placed in a pellet die and compressed with 10 tons of pressure for one grinding cycle to form a pellet.

\begin{tabular}{|l|l|l|l|l|l|l|l|l|l|l|l|}
\hline & Mix1 & Mix2 & Mix3 & Mix4 & Mix5 & Mix6 & Mix7 & Mix8 & Mix9 & Mix10 & Mix11 \\
\hline Graphite & 0.0770 & 0.3344 & $0 \mathrm{~g}$ & 0.0082 & 0.0936 & 0 & 0.1519 & 0.2259 & 0.1757 & 0 & 0 \\
\hline NIST1635 & 0.0704 & 0 & 0.7575 & 0.3113 & 0.2985 & 0.2967 & 0.2415 & 0.2121 & 0.2715 & 0.7141 & 0.6357 \\
\hline $\begin{array}{l}\text { Iron } \\
\text { Powder }\end{array}$ & 0.0138 & 0.0060 & 0.0998 & 0.0199 & 0.0213 & 0.0091 & 0 & 0.0017 & 0.0072 & 0.4141 & 0.0027 \\
\hline $\begin{array}{l}\text { Mercuric } \\
\text { Oxide }\end{array}$ & 0.0108 & 0.0026 & 0.0111 & 0.0019 & 0.0003 & 0.0084 & 0.0132 & 0.1497 & 0.0138 & 0 & 0 \\
\hline
\end{tabular}

Table 3.1 - Synthetic Mixtures

\begin{tabular}{|l|l|l|l|l|l|l|l|l|l|l|l|}
\hline & Mix1 & Mix2 & Mix3 & Mix4 & Mix5 & Mix6 & Mix7 & Mix8 & Mix9 & Mix10 & Mix11 \\
\hline Carbon, \% & 73.42 & 97.49 & 61.06 & 66.24 & 73.12 & 67.75 & 81.20 & 81.21 & 78.11 & 65.93 & 69.73 \\
\hline Iron, \% & 8.12 & 1.75 & 11.70 & 6.05 & 5.32 & 3.18 & 0.15 & 0.46 & 1.68 & 6.04 & 0.62 \\
\hline Mercury, \% & 5.81 & 0.70 & 1.18 & 0.52 & 0.08 & 0.25 & 0.31 & 2.77 & 2.74 & 0 & 0 \\
& & & & & & & & & & & \\
\hline
\end{tabular}

Table 3. 2 - Elemental Concentrations of Synthetic Mixtures

\subsection{LIBS Experiment}

\subsubsection{Modular Collimator System}

Collection Optics determined how much light was fed into the spectrograph. The CCD camera could process greater emission signals with more light, optimizing the 
detection of trace elements such as mercury. Light emitted from all directions during plasma formation. The optics were selected to transmit optimum UV and visible light while avoiding aberrations.

The image was made to be small enough to fit several times on the y-axis of the CCD camera for dynamic LIBS testing. A spatial filter was built to reduce the spark size. The final design of the spatial filter included a series of $f / 4$ lenses $(f=100 \mathrm{~mm}, d=5.4 \mathrm{~mm})$ for collection and focusing down to the pinhole. A smaller focal length lens ( $\mathrm{f}=35 \mathrm{~mm}$, $\mathrm{d}=25.4 \mathrm{~mm}$ ) was placed after the pinhole to reduce image size and avoid eclipsing of the beam incident on the scanning mirror facets. The modular collimator system allowed light from the laser spark to be collected, focused through a pinhole and collimated before reaching the scanning mirror

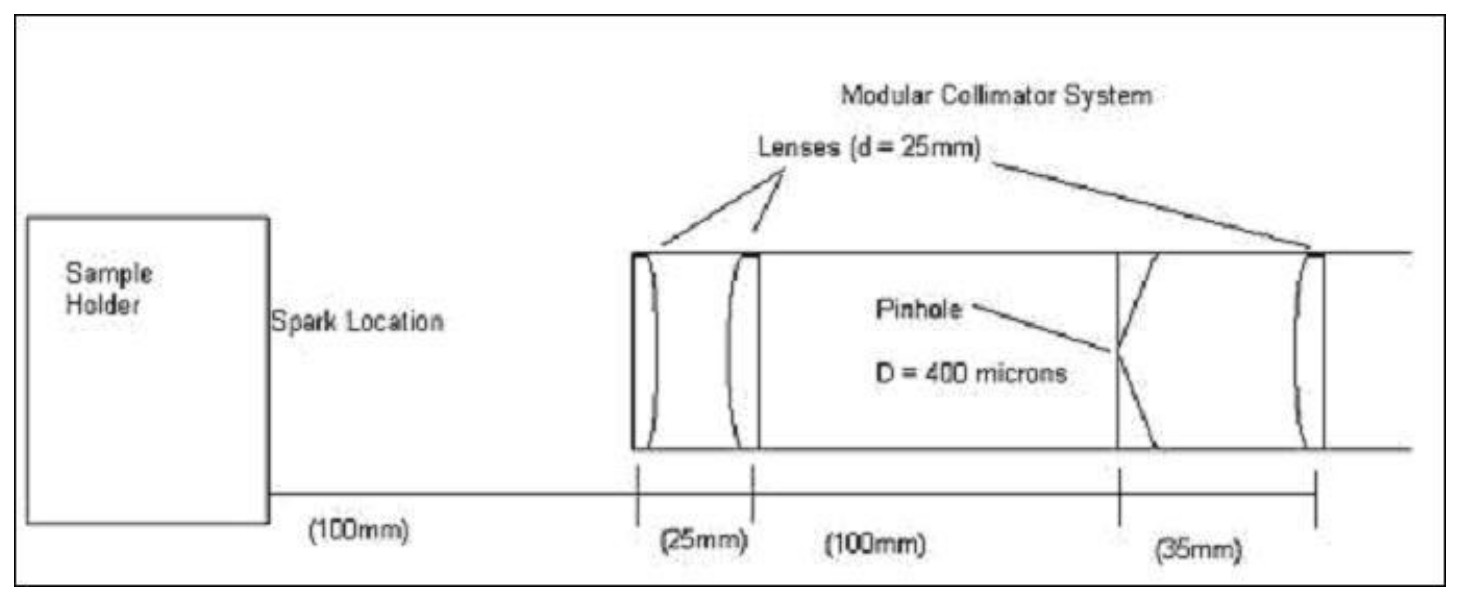

Figure 4. 1 - the dimensions of the Modular Collimator System

\subsubsection{Spectrograph Selection}

During LIBS testing, several wavelengths were investigated to determine the region of interest. In other experiments, elements have been known to interfere with each other. Iron was known to interfere with carbon [16] and mercury [15] with shorter focal length spectrographs. A high-resolution spectrograph (HR 640 from ISA - Jobin Yvon) 
was used with an 1800 grove/mm grating. This allowed a resolution of 0.02 nanometers, where a LIBS experiment that would use $250 \mathrm{~mm}$ focal length or $500 \mathrm{~mm}$ focal length spectrographs and have a resolution of $0.3-0.05$ nanometers. With greater resolution, emission lines that regularly interfere with each other could be resolved. The wavelength range would encompass 20 nanometers at a time. A search was conducted to find wavelengths characteristic to the elements of interest that were within 20 nanometers of each other.

\subsubsection{Experimental Setup}

The experimental LIBS apparatus was designed and built to allow optimum signal collection as well as temporal characteristics of the LIBS spark of coal. A two channel pulse generator (BNC 555) was used to control the synchronization of the experiment. When the pulse generator was activated, channel A sent a signal to the flash lamp of the laser (Quanta Ray DCR 11 Q-switched Nd: YAG). Approximately 250 microseconds after the signal was sent to the flash lamp, the laser fired. The laser beam was focused through a focusing lens (CVI Laser, focal length $=100 \mathrm{~mm}$, diameter $=25.4 \mathrm{~mm})$ onto a pellet, creating a plasma spark. The CCD controller was connected to output channel B of the pulse generator. 


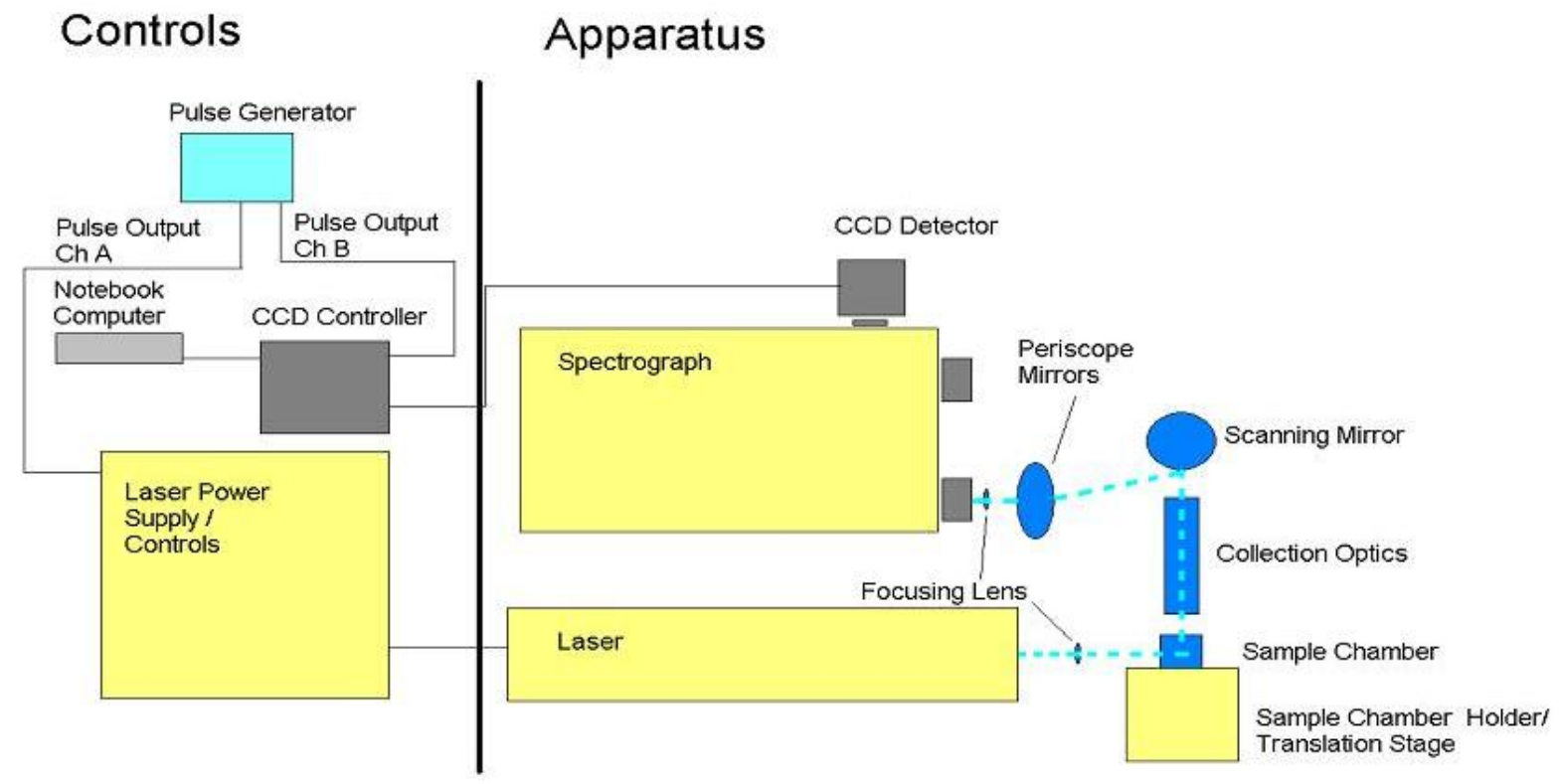

Figure 3. 2 - LIBS Testing Schematic

Once the laser spark occurred, the light was collected and collimated by a modular collimator system. The collimated beam traveled to the polygonal scanning mirror (Lincoln Laser Company), where it was reflected off one of the scanning mirror facets. The beam then traversed through a set of periscope mirrors, and was reflected onto a final focusing lens (focal length $=50 \mathrm{~mm}$, diameter $=25.4 \mathrm{~mm}$ ). The lens focused the collimated light onto the entrance slit to the spectrograph (Horiba Jobin-Yvon HR640). The use of the scanning mirror in the experiment determined the type of LIBS test.

\subsubsection{Static LIBS and Dynamic LIBS}

When the scanning mirror was turned off, data from the LIBS spark was taken with no internal gating. Also, because of a lack of a shutter on the CCD camera, the CCD camera was fully exposed until the data was completely read out, which was approximately 2.6 seconds. The image formed by the spark created a spot on the 
entrance slit. This spot encompassed the entire lifetime of the laser-induced plasma, from the black body radiation observed in initial plasma formation to the emission lines resulting from the recombination of atoms and molecules. This process was deemed "static LIBS".

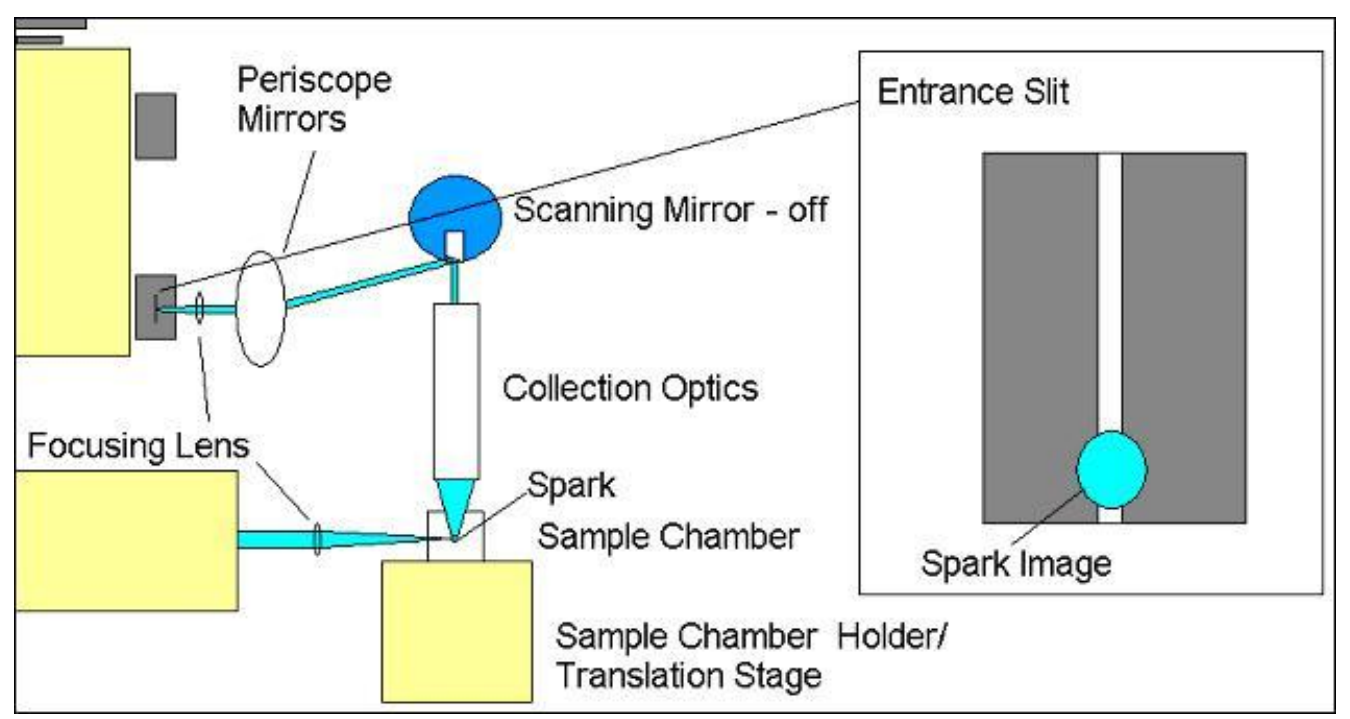

Figure 3. 3 - "Static" LIBS

When the scanning mirror was enabled, the image of the spark was spread out across the entrance slit because of the mirror's motion. This created a line on a portion of the entrance slit. Each point in the line represented a different time within the lifetime of the spark. This information provides us with an approximate time frame to observe the temporal behavior of each emission line. This process was named "dynamic LIBS". 


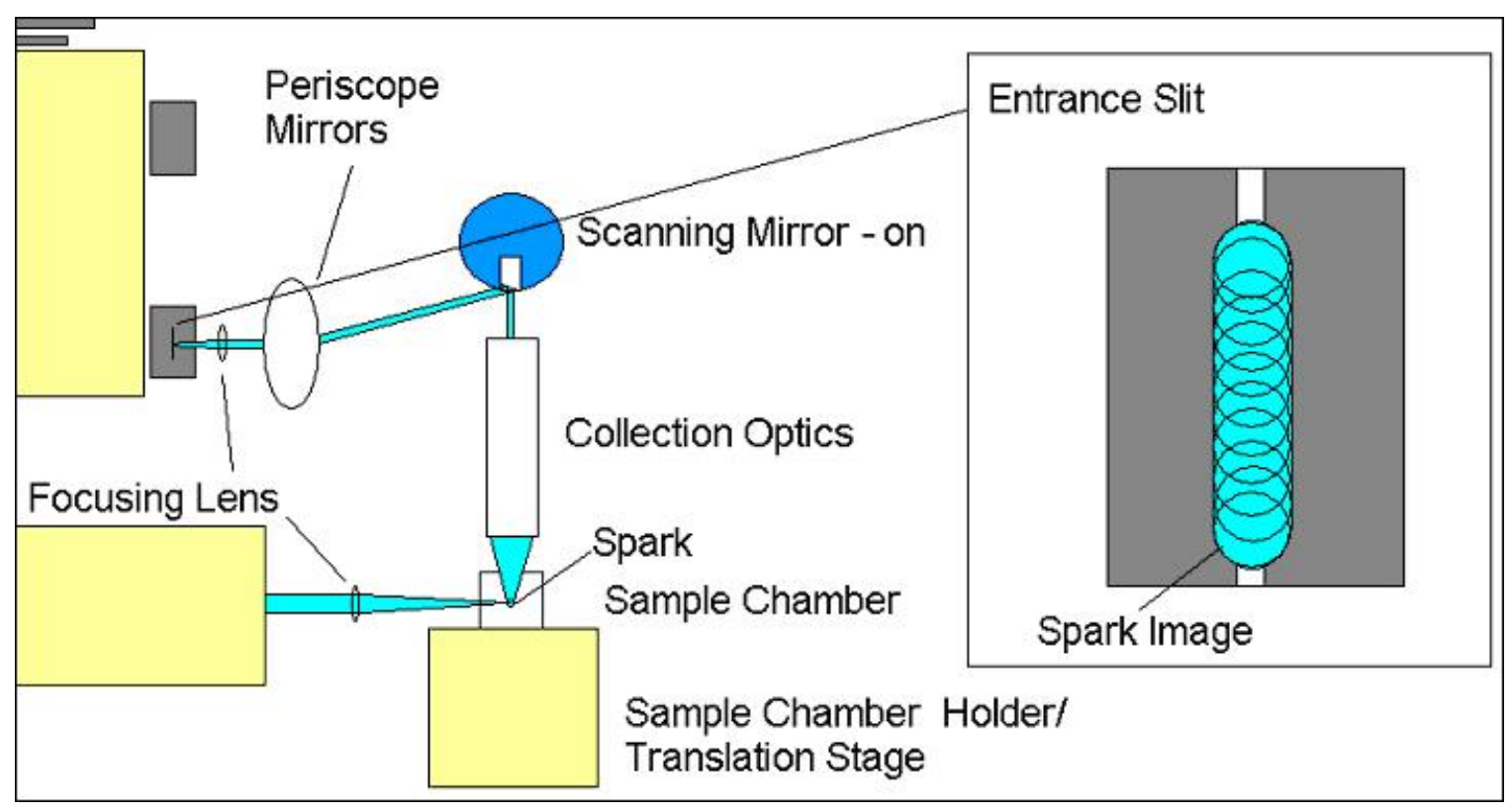

Figure 3.4 - "Dynamic” LIBS

\subsubsection{LIBS Testing Procedure}

During startup, the laser, computer, detector controller and pulse generator were turned on, the overhead ceiling light (which contained mercury) was turned off, and the software (Winspec32) was opened. Channel B on the pulse generator was delayed by 250 microseconds compensate for the delay time between the pulse to the flash lamp from channel A and the actual laser firing.

The specimen holder with a mercury lamp was aligned to the optics via a translation stage. The mercury lamp emitted light through a pinhole $($ diameter $=250 \mu \mathrm{m})$ at the approximate spark location. The mercury lamp, because of its intensity, made an excellent alignment tool.

During spectrograph calibration, which followed alignment, the pinhole was removed and replaced with a sample chamber, which was a 2"x2"x2" cube constructed 
in-house using components from Edmund Optics and Mountaineer Glass \& Mirror Company. The sample holder contained iron powder for calibration of the spectrograph.

To perform spectrograph calibration, a LIBS spectra of iron powder with the ceiling lights on was obtained to observe iron and mercury emission lines. The spectrograph was then tuned to a selected wavelength range, and calibrated using a linear relationship between known emission lines of iron and mercury. This step was only necessary when selecting the desired wavelength range to be observed.

Once the calibration specimen was removed, the pinhole was placed over the lamp source and the alignment was repeated. After re-alignment, the pinhole was removed and a sample chamber with a test specimen was placed in the sample holder.

The spinning mirror was either turned on or left off, depending of the desired test. The mirror was left off for static LIBS testing, and turned on for dynamic LIBS testing. Pulse generator triggered the flash lamp to the laser (channel A) as well as the CCD controller (channel B). The collected light traversed through the pinhole into the modular collimated system. If the light from the spark did not go through the pinhole, then the sample holder was appropriately translated until the light collected from plasma emission was focused through the pinhole. For dynamic LIBS testing, the spinning mirror functioned independently from the pulse generator and was not synchronized to the rest of the apparatus, resulting in a hit or miss method to acquire data. 


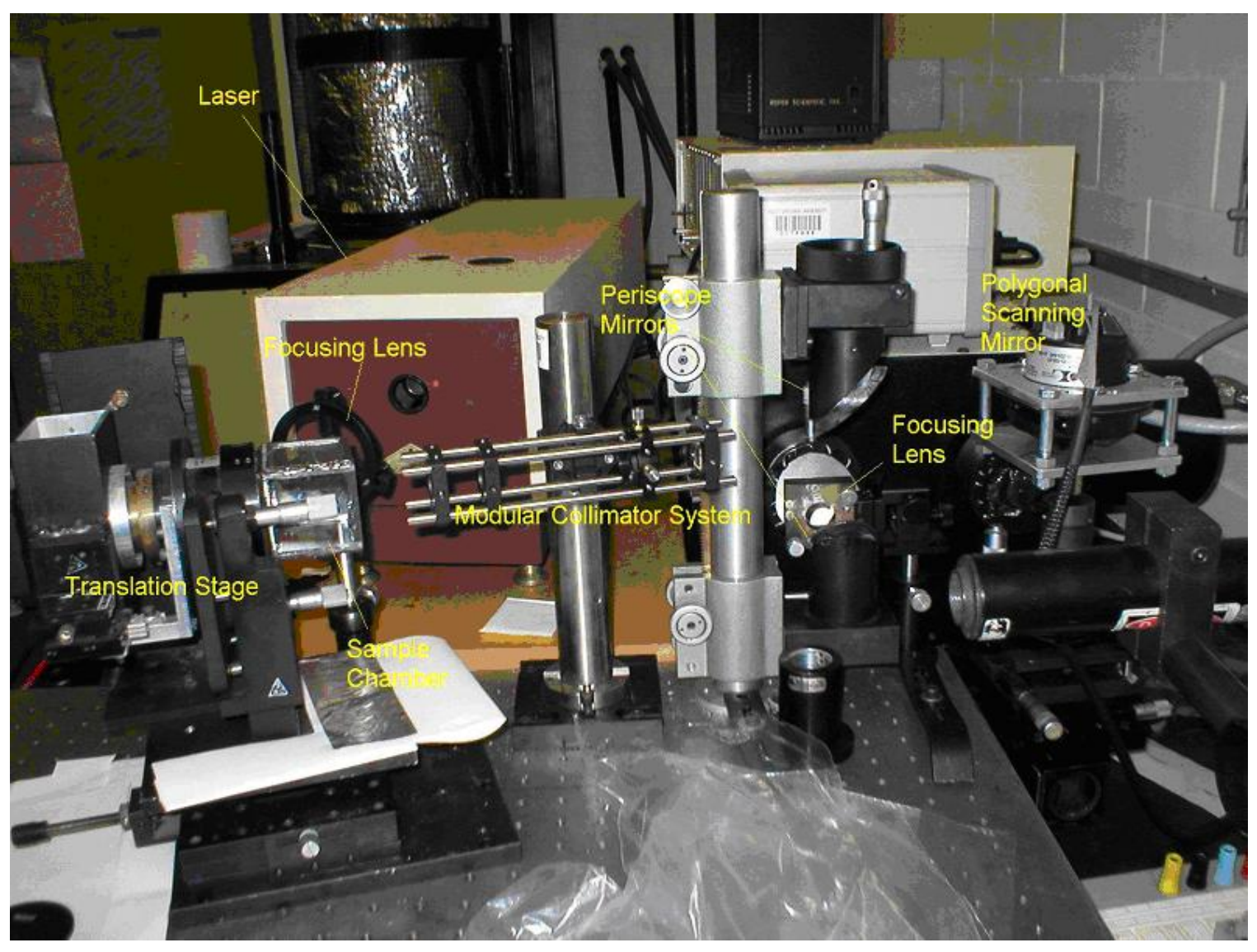

Figure 3. 5 - LIBS Testing Apparatus

\subsubsection{File Processing Procedure}

Before any data files can be analyzed, they were processed into a form that was useful for data processing. The raw data file was opened in Winspec32, where it was then binned. Binning is a process in spectral analysis software that sums the vertical pixels of the CCD and reduces the noise when compared to the signal of the emission lines. Binning of the emission spectrum involved the summation of all 252 active pixels along the vertical axis. The binned signal was then converted into Grams/AI by a seamlessly integrated conversion. After the signal was converted to Grams/AI, it was then converted into a Microsoft Excel file through a program in Grams/AI called "Excel 
Exchange”. A graphical run through of the steps for file processing is explained in further detail in Appendix A.

The data processing for dynamic LIBS was a completely different procedure. While static LIBS testing determined the elemental composition of the specimen, dynamic LIBS testing observed time-dependent characteristics of the individual emission lines within the specimen. Data processing for dynamic LIBS involved a process in WinSpec32 called cross-sectioning. A "good" LIBS shot was selected for cross sectioning. A "good" LIBS shot was a single shot spectrum that displayed all six emission lines of interest (Fe 432nm, Hg 436nm, Fe 439nm, Fe 441nm, C 445nm, and C 447nm) and showed no dominant continuum radiation. Each emission line of interest in the LIBS spectrum was individually selected and subject to the cross-sectioning process (explained in appendix A). 


\section{CHAPTER 4: RESULTS AND DISCUSSION}

\subsection{Initial LIBS Testing}

Initial testing for signal acquisition and optimization began with steel. This choice was made because iron has a rich spectrum, and can be observed throughout the spectral regions of interest, making it an optimal candidate for spectrograph calibration. Both static and dynamic LIBS testing were applied to steel.

The first studies were observed in the wavelength range of 530-545nm. This wavelength range included at least one emission line from each of the elements of interest was anticipated (3 sulfur lines, 1 carbon line and 1 mercury line). Static LIBS was applied to pyrite, sulfur powder, coal powder and graphite powder. No signals from carbon or sulfur were observed in this wavelength region. Pyrite testing only revealed iron emission lines. Mercury was not tested in this region.

The spectrograph was tuned to mercury's most sensitive line, 254nm. In this range, the spectrograph would encompass the $254 \mathrm{~nm}$ line from mercury as well as the 248nm line in carbon. LIBS testing was done on the coal sample from NIST, the expected carbon line was not observed in this range, nor was mercury.

In an attempt to find sulfur, the spectrograph was tuned to the wavelength range to observe emissions at 921, 923, and 928nm. Static LIBS testing was applied to a sulfur pellet. Experiments done to detect sulfur were also conducted with the spectrograph centered at sulfur's $496 \mathrm{~nm}$, and $180 \mathrm{~nm}$ line. Sulfur showed no emission lines from the ultraviolet to the infrared spectrum. It is hypothesized that this could be due to effects of an air environment that seem to quench sulfur emission lines. 
The spectrograph was tuned to the wavelength range of 431-452nm. Static LIBS testing was done on coal powder, coal pellets, graphite powder, graphite flakes, a carbon rod, carbon powder, sulfur powder, sulfur pellets, iron powder, and a mercury-copper amalgam. Upon testing the powders, no emission lines were observed in graphite or sulfur. LIBS testing applied to coal powder revealed emission lines at approximately 445 $\mathrm{nm}$ and $447 \mathrm{~nm}$, which were tested against a carbon rod and matched. These wavelengths were also observed in LIBS testing applied to pure carbon powder. The 436nm mercury emission line was observed in the testing of the mercury-copper amalgam. Only iron emission lines appeared in the LIBS testing of the pyrite sample, no sulfur emission lines were observed. Since all carbon, iron and mercury were present within this wavelength range, this wavelength range was chosen for LIBS experiments on synthetic mixtures.

\begin{tabular}{|l|l|l|l|l|l|l|l|l|l|l|}
\hline & \multicolumn{2}{|c|}{ COAL } & \multicolumn{2}{c|}{ GRAPHITE } & \multicolumn{2}{c|}{ CARBON } & \multicolumn{2}{c|}{ SULFUR } & IRON & MERCURY \\
\hline LINE & POWDER & PELLET & POWDER & FLAKES & POWDER & ROD & POWDER & PELLETS & POWDER & AMALGAM \\
\hline FE432 & & & & & & & & & $x$ & \\
\hline HG436 & & & & & & & & & & \\
\hline FE439 & & & & & & & & & $x$ \\
\hline FE441 & & & & & & & & & $x$ \\
\hline C445 & $x$ & $x$ & & & $x$ & $x$ & & & $x$ \\
\hline C447 & $x$ & $x$ & & & $x$ & $x$ & & & \\
\hline
\end{tabular}

Table 4. 1 - Emission Lines Observed in 431-452nm wavelength range.

\subsection{LIBS Testing of Synthetic Mixtures}

Static and dynamic LIBS testing were applied to the synthetic mixtures. To compensate for the lack of uniformity in the samples, 100 static LIBS shots were taken for each synthetic mixture. Standard deviation values of the shot intensities of each sample indicate that the mixtures were not thorough. After static testing of a specimen, 
the spinning mirror was then turned on for dynamic testing. Dynamic shots were collected from each sample, except for sample \#2, which was destroyed during static testing.

\subsection{Static LIBS Analysis}

\subsubsection{Qualitative Analysis}

Qualitative analysis of the LIBS signals can be done with the data of the static LIBS shots. Emission lines were visually observed by identifying their maximum intensity values, and correlated to the calibration of the spectrograph for identification. Iron was identified at 432nm, 439nm, and 441nm. Mercury was identified at 436nm. Two emission lines at 445 and 447 were observed to be carbon-related emission lines.

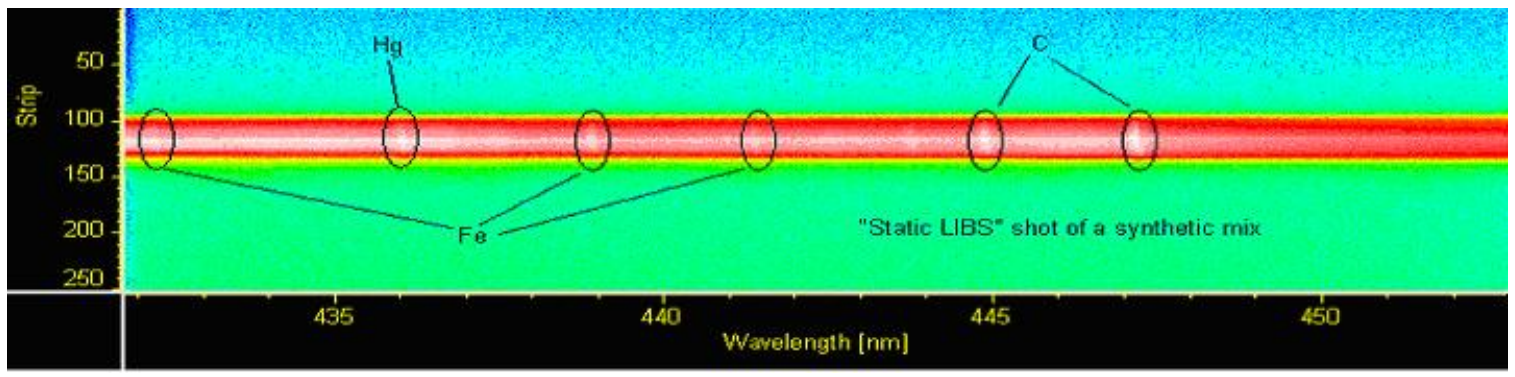

Figure 4. 2 - Static LIBS Raw Data, mix\#1, shot 59

\subsubsection{Quantitative Analysis}

The data from 100 static shots of each sample was signal averaged for quantitative analysis. In this procedure, spectral background was subtracted from the signal (which included electronic response of the detector and the continuum radiation from the initial plasma formation).

$$
\mathrm{I}_{\text {emission }}=\mathrm{I}_{\text {signal }}-\mathrm{I}_{\text {background }} \ldots(4-1)
$$


Where $I_{\text {signal }}$ is the intensity at the peak of the emission line, and $I_{\text {background }}$ is the intensity at the baseline of the emission line. Because of the initial radiation resulting in a broad emission that interferes with the atomic and ionic emission signals, the baseline intensities to the left and right of the emission lines were averaged before subtracting the baseline value from the peak intensity. The intensity of the emission lines resulting from LIBS were plotted with respect to the elemental concentration of the corresponding specie within the sample. Emission lines suitable for analytical purposes display linear relationship between intensity and elemental concentration.

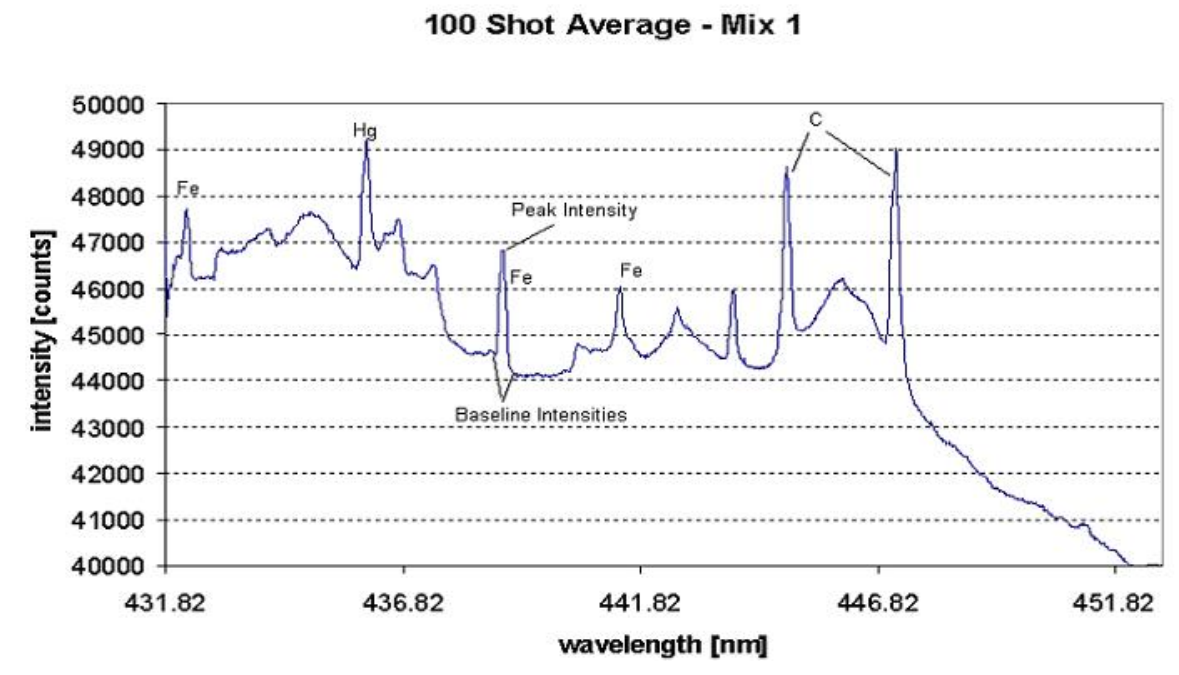

Figure 4. 3 - 100 Shot Average of Mix 1 for qualitative and quantitative analyses 


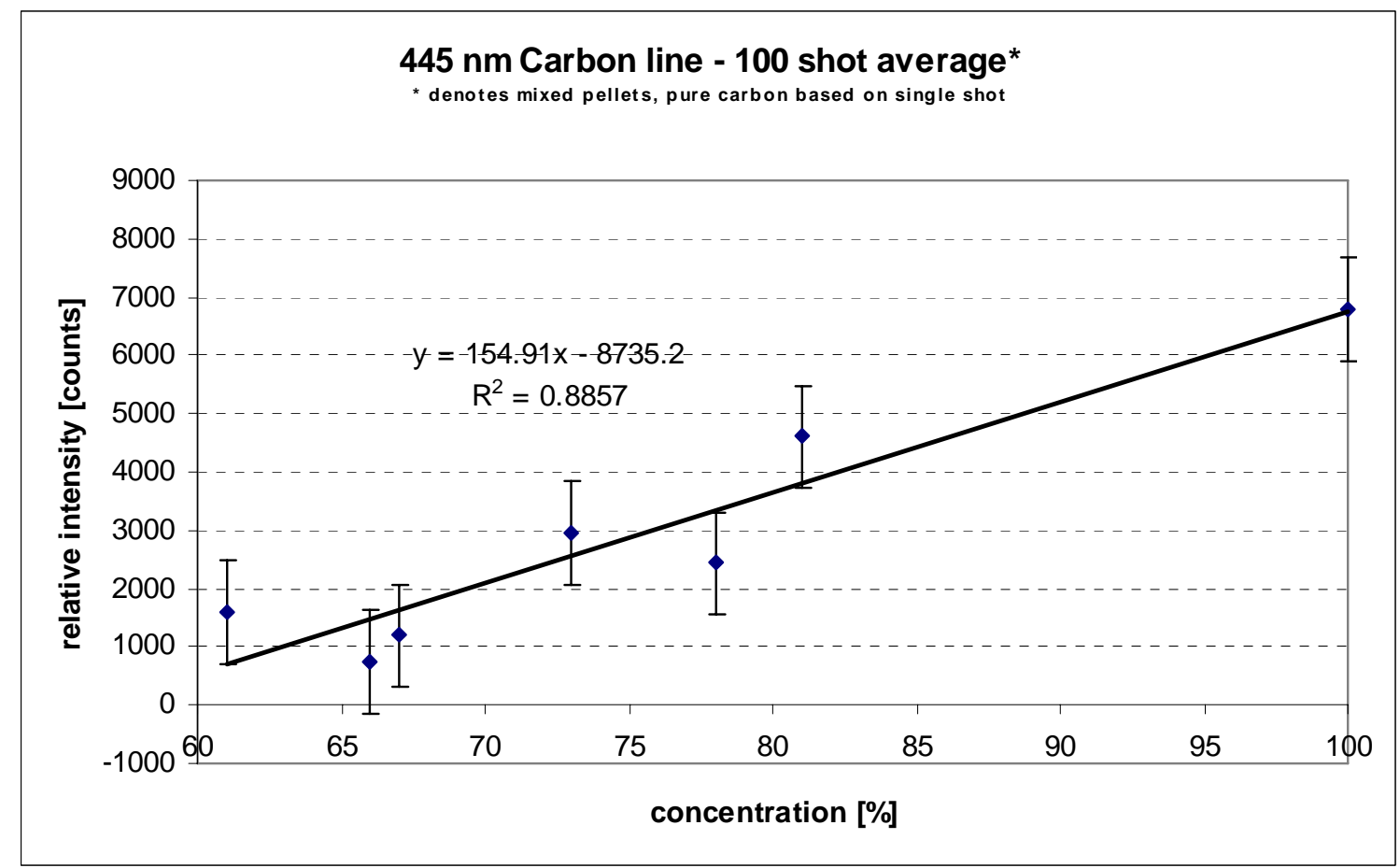

Figure 4. 4 - Correlation of the 445nm Carbon - related line

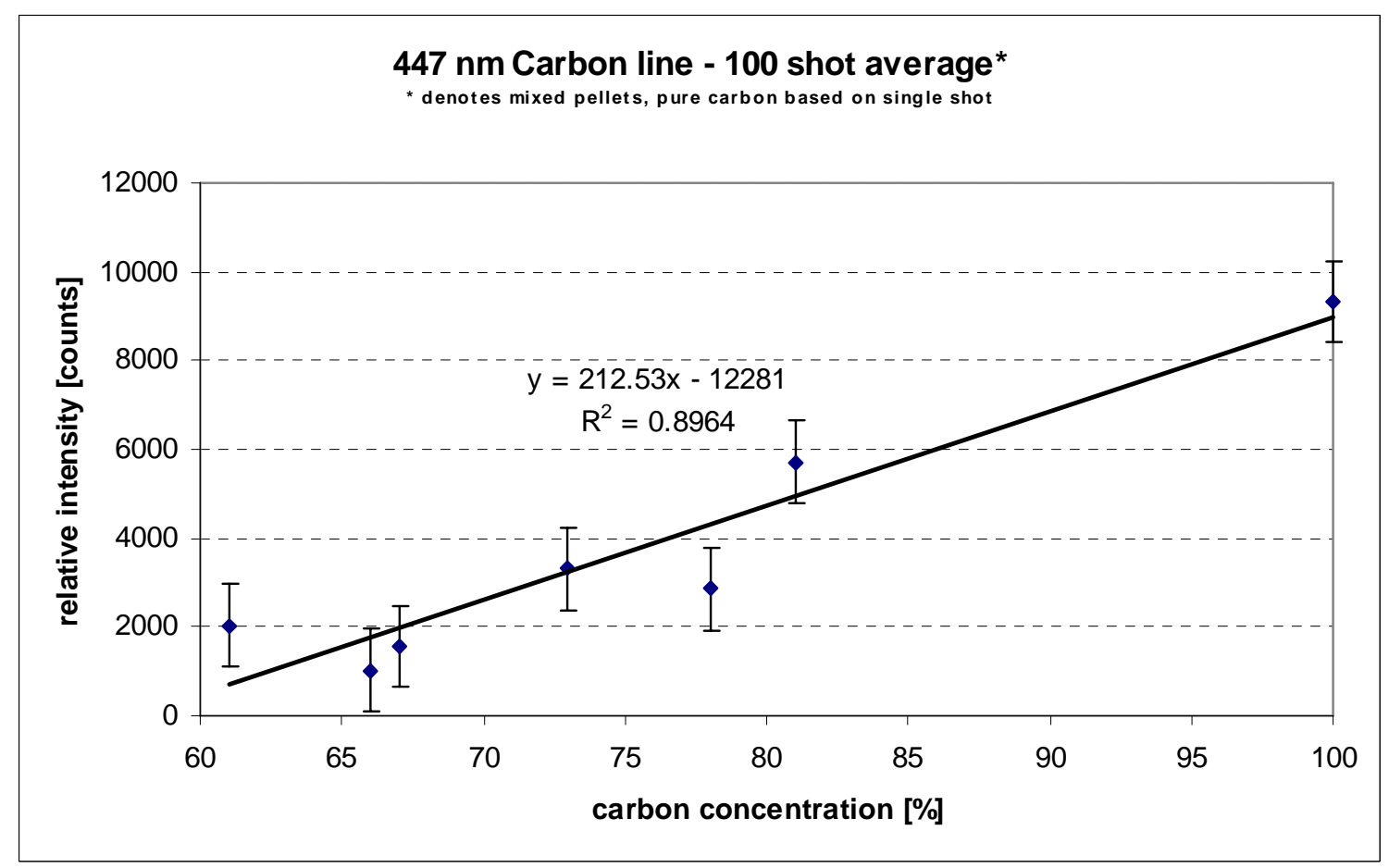

Figure 4. 5 - Correlation of the $447 \mathrm{~nm}$ Carbon - related line 
The linear fit observed in the 445 and $447 \mathrm{~nm}$ carbon-related lines showed linear fit between elemental concentration and line intensity at higher concentrations. These emission lines were not tested at concentrations lower than 60 percent by mass. A forecast through their trend line would show a non-zero intercept, and therefore, do not have strong correlation to concentration for analytical purposes.

Mercury concentration ranged between 800 parts per million to 6 percent. The strongest and most observed line is 253.6 [15, 21, 23]. The 253.6nm mercury line was not observed during initial LIBS testing. It is hypothesized that the 253.6nm mercury line may have been quenched by standard atmospheric conditions. The 436nm emission line was chosen because of its close proximity to the carbon-related and iron emission lines. The $436 \mathrm{~nm}$ emission line proved to have a linear relation between line intensity and elemental concentration. There was one discrepancy point within the data set, which is hypothesized to be a measurement error that occurred during the pellet formation process. 


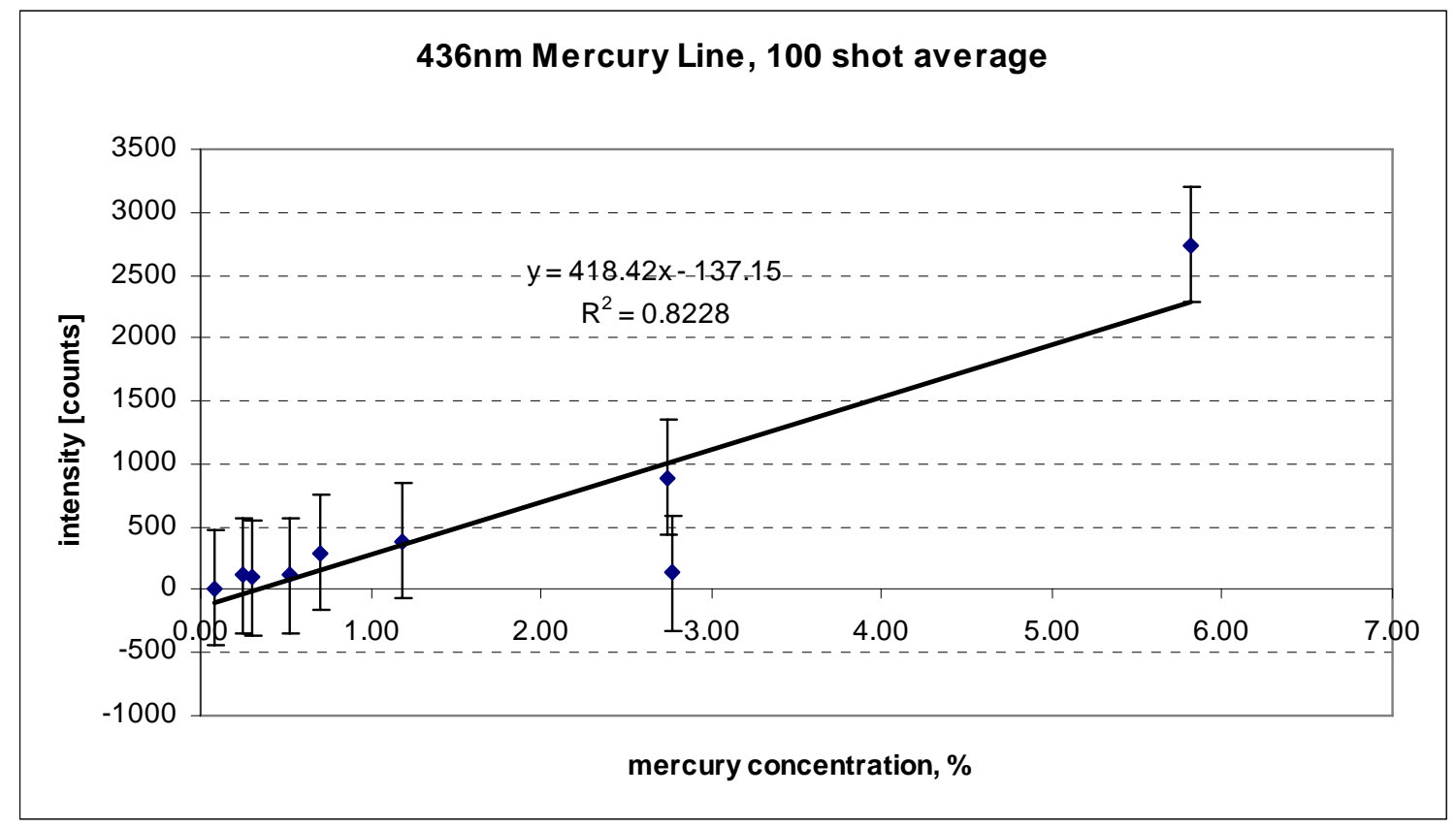

Figure 4. 6 - Correlation of the 436nm Mercury Emission Line

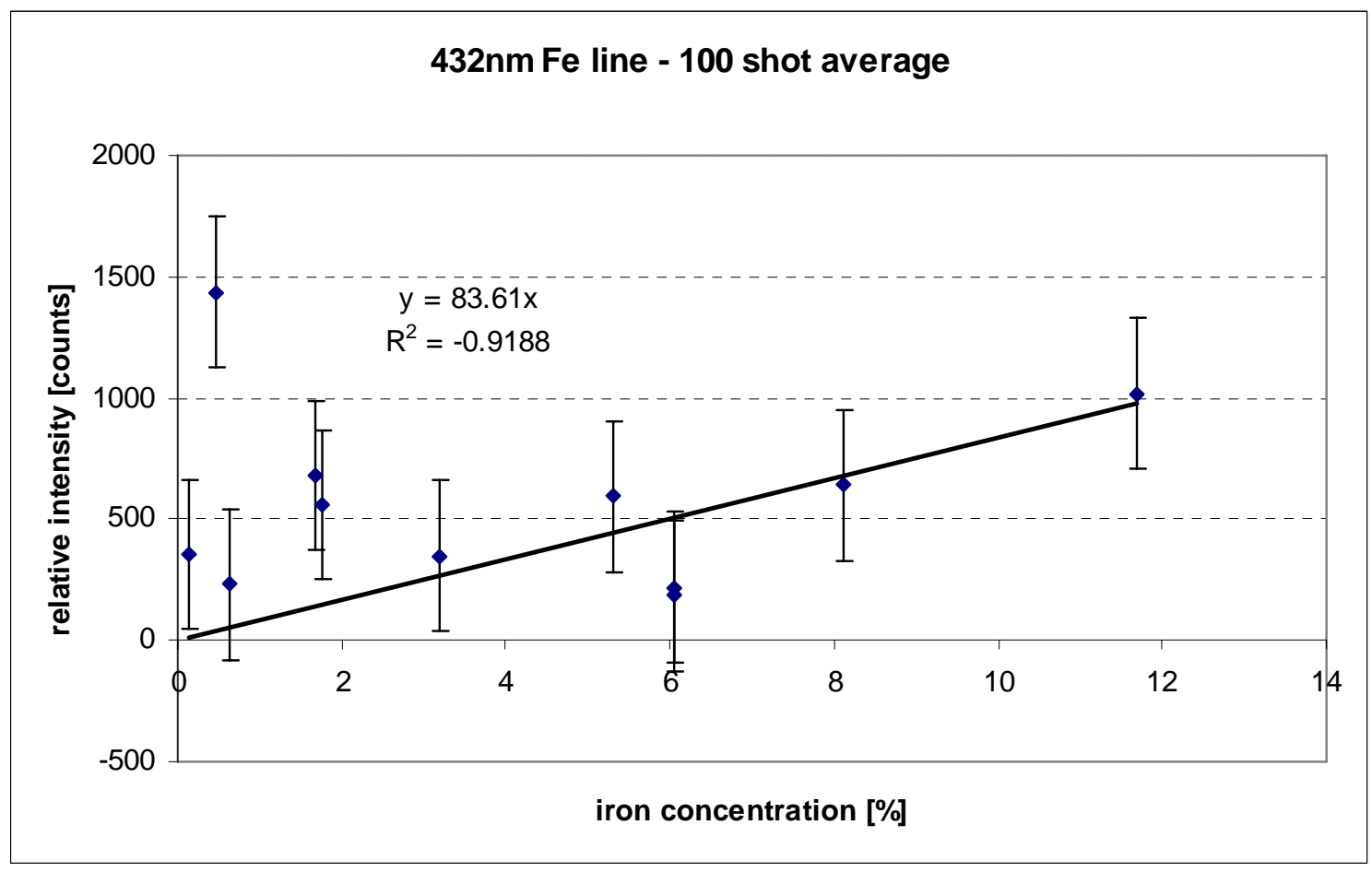

Figure 4. 7 - Correlation of the 432nm Iron Emission Line 


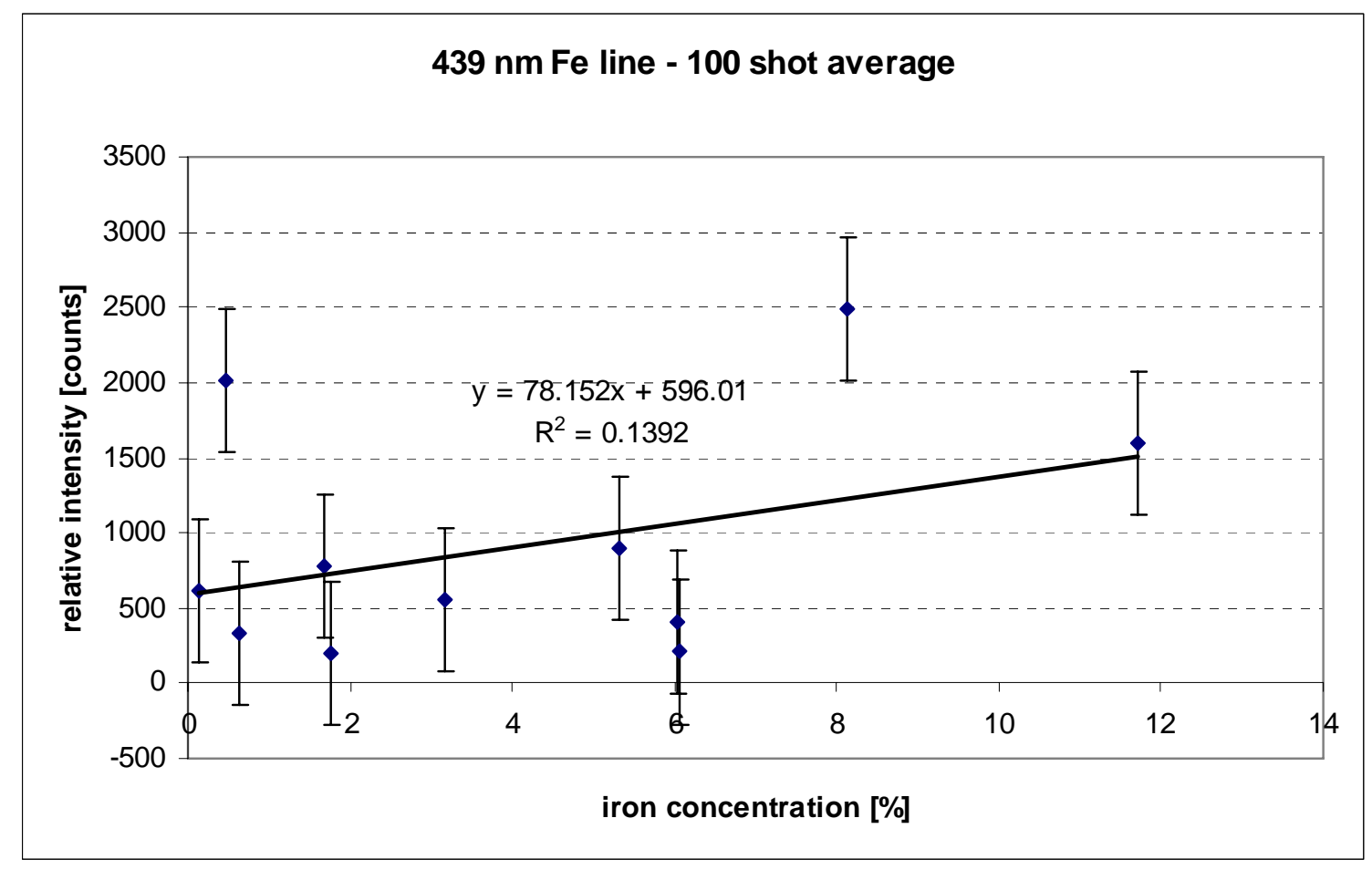

Figure 4. 8 - Correlation of the 439nm Iron Emission Line

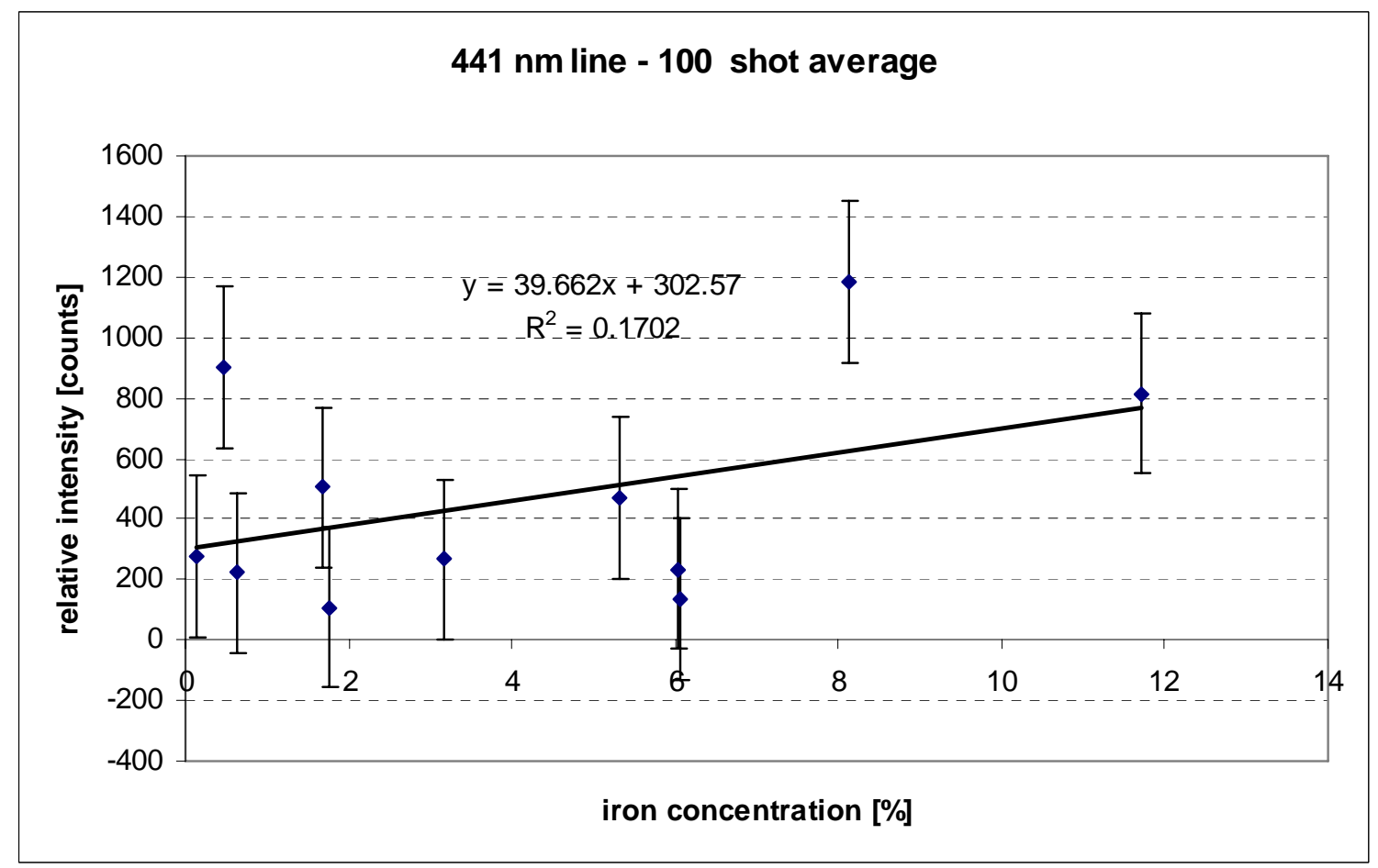

Figure 4. 9 - Correlation of the 441nm Iron Emission Line 
The iron lines showed a poor correlation between emission line intensity and elemental concentration. The iron found in coal is mostly mineral forms of iron, such as iron oxide [4]. The iron used to make the synthetic mixtures was pure iron powder. It is hypothesized that the iron powder formed together when subject to the grinding unit, which created small areas of heavy iron concentration within the heterogeneous mixture.

\subsection{Dynamic LIBS Analysis}

\subsubsection{Dynamic LIBS}

Since the LIBS spark is brief, it is necessary to have a time frame in which the characteristic line spectra can be observed. In their review, "Quantitative micro-analysis by laser-induced breakdown spectroscopy: a review of the experimental approaches", Tognoni et. al. state that early stages of the laser-induced plasma will contain black body continuum radiation [26]. The continuum correlates to the emission of free electrons during initial plasma formation and cannot be reduced through averaging, though it can be normalized though use of Wien's Law. The best way to compensate for the continuum emission is to activate the spectral acquisition with a proper delay after the laser-induced plasma has been produced. So far, LIBS experiments have been temporally resolved by the use of an Intensified CCD (ICCD). The ICCD allows the option of temporal gating. This means that the end user of the equipment can set a delay time after the black body continuum has been reduced and during an optimal time for observing the characteristic line spectrum of the element in question.

The dynamic LIBS method was developed to observe temporal behaviors of multiple emission lines in a single LIBS spectrum. This system incorporated the use of a 
polygonal scanning mirror to achieve temporal differentiation within the emission spectrum. To current knowledge, this was the first time a polygonal scanning mirror was applied to a LIBS experiment for time-resolved studies. The polygonal scanning mirror reflected light off of one of its facets, creating a line image instead of a spot image of the LIBS spark. Each segment of the line will correspond to a different time. The scan line fell on the entrance slit of the spectrograph. The light fell on the CCD array in an area that correlated to a temporal profile, creating a temporally resolved, as well as a spatially resolved LIBS signal.

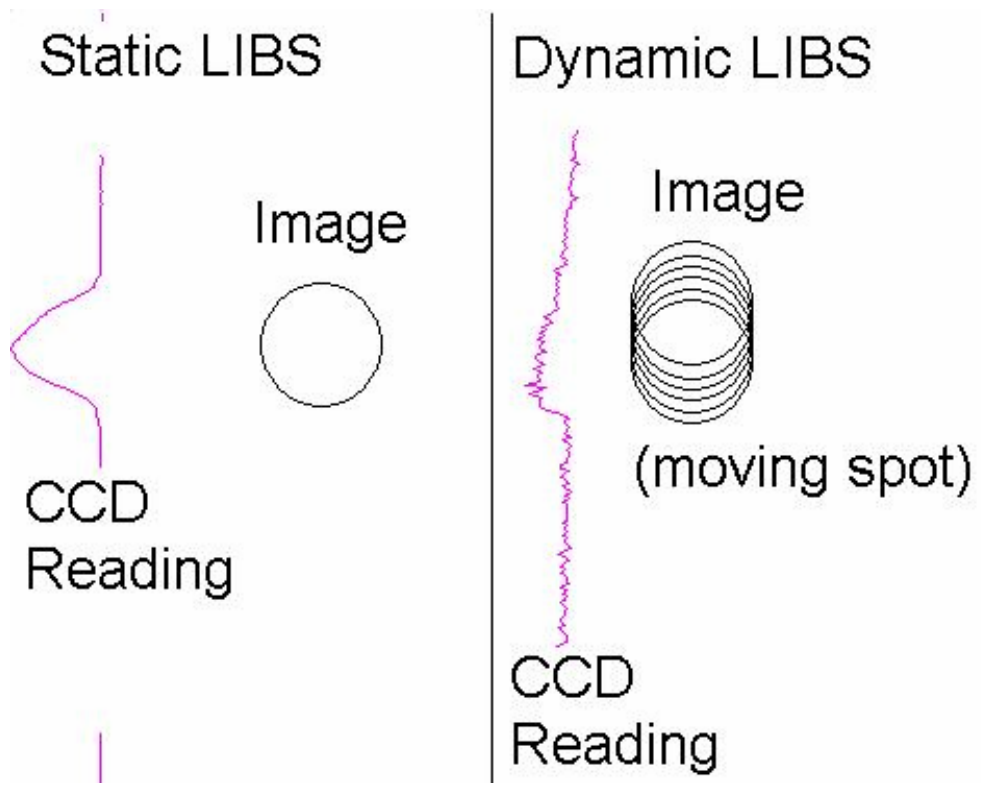

Figure 4. 10- Images and Intensity Profiles of Static and Dynamic LIBS

\subsubsection{Calculation of the Dynamic LIBS Time Frame}

The mirror used was a 30 faceted polygon spinning at 12,000 rpm. The spinning of the scanning mirror allowed for a time-dependent signal to be generated spectrograph's entrance slit. The dimensions for each facet were $9.8 \mathrm{~mm}$ high by $6.0 \mathrm{~mm}$ wide. The incident light on the facet was a circle with a diameter $8.9 \mathrm{~mm}$. The scanning 
mirror facet reflected the light, changing the angle incident on the focusing lens, resulting in the incident light imaging to a line instead of a point. The line image across the CCD array displayed the temporal characteristics of the LIBS spark.

The number of facets and the rotation speed in the scanning mirror determined the time frame in which the laser spark was observed. The scanning mirror had 30 facets around the circumference of a disk, which resulted in a 12 degree scan angle for each facet. The rotation speed of the scanning mirror was set at 200 revolutions per second. The value in revolutions per second was converted into degrees per second by multiplying by 360 degrees per revolution i.e. 72,000 degrees per second. The full 12 degree scan of an individual mirror facet would take 167 microseconds. The focusing lens did not include the full scan of the mirror facets. The optical path from the scanning mirror to the focusing lens was $330 \mathrm{~mm}$. For the full scan of a facet to be encompassed, the lens diameter would have to be $62.6 \mathrm{~mm}$. The diameter of the focusing lens was 25.4mm, resulting in a reduced scan angle, and therefore, a reduced time frame of observation.

To calculate the window of observation, the beam was modeled in OSLO, an optical modeling software. From the OSLO modeling, the length of the line incident on the entrance slit of the spectrograph was calculated to be 2 millimeters. The operational scan angle was calculated to be 2 degrees. Figure 4.10 depicts the beam path of the light through the final focusing lens at the center and at a scan angle of 1 degree. The scan distance is 1 millimeter, making the spot size $2 \mathrm{~mm}$ on the entrance slit to the spectrograph. 


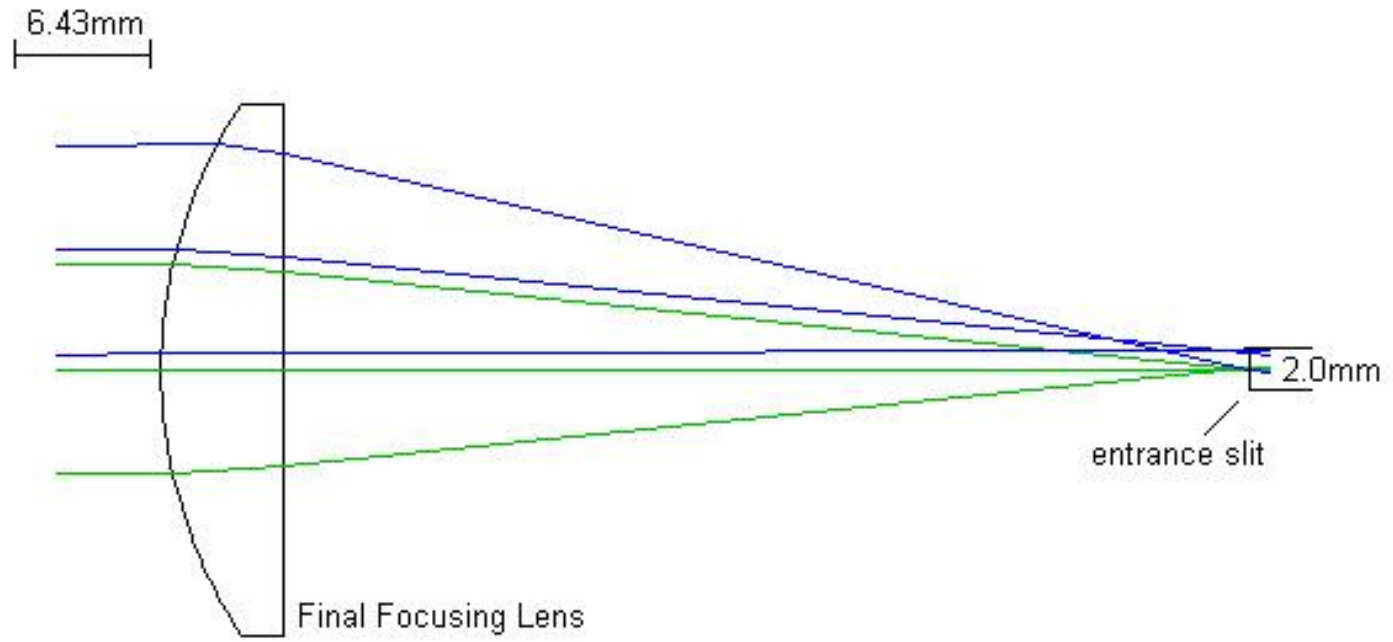

Figure 4. 11 - Modeling in OSLO. The spark image was scanned to form a $2 \mathrm{~mm}$ line on the entrance slit to the spectrograph.

From the rotation speed of the scanning mirror and the number of degrees per incident scan, the observation window was calculated at 27.8 microseconds. The size of the incident line (2mm) was used to calculate the region of the CCD used. The CCD has 256 pixels and is $6 \mathrm{~mm}$ high. The incident line from the laser spark was $2 \mathrm{~mm}$ high. Of the 256 pixels on the CCD, 86 pixels were actively collecting light from the laser spark. The time scale for the dynamic LIBS experiments was calculated to be 0.32 microseconds per pixel. 


\subsubsection{Signal Processing and Analysis}

The dark current in the dynamic LIBS data was normalized before further signal processing could take place. The dark current response of the CCD was added to the dynamic LIBS signal. The use of an exponential fit to the dark current was subtracted from the original signal to eliminate the dark current from the dynamic LIBS signal.

HG 436

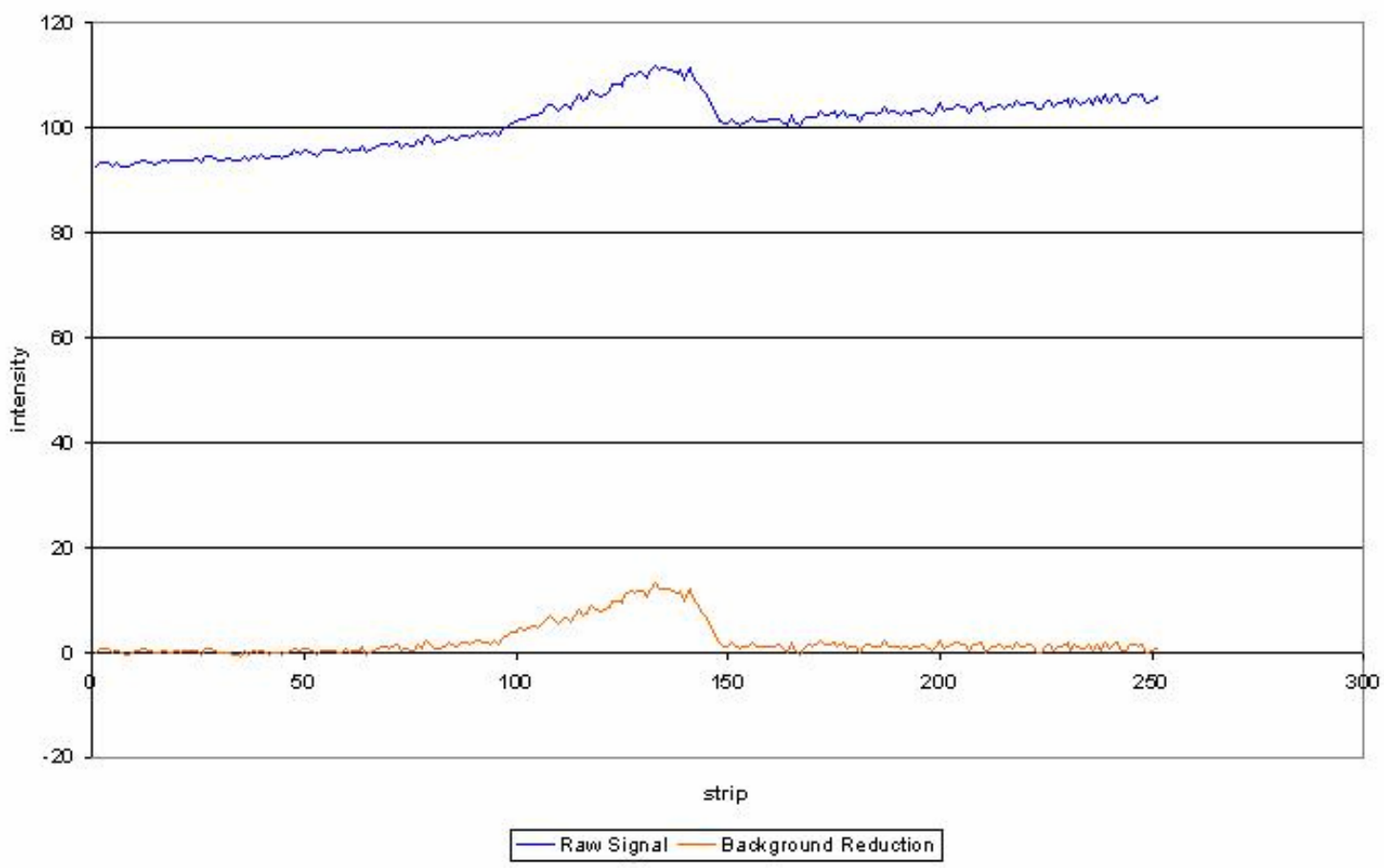

Figure 4. 12 - Original and Background Corrected Signals

The final focusing lens of the experimental set up eclipsed the dynamic LIBS signal. The eclipsing convoluted the signals of the true time-dependent characteristics of the emission lines. To recover this data, a function had to be formulated to de-convolute the effects of the eclipsing. The eclipsing of the beam and the lens was modeled in AUTOCAD. The modeling process calculated the areas of the incident beam of the laser spark as it moved off of the final focusing lens. 


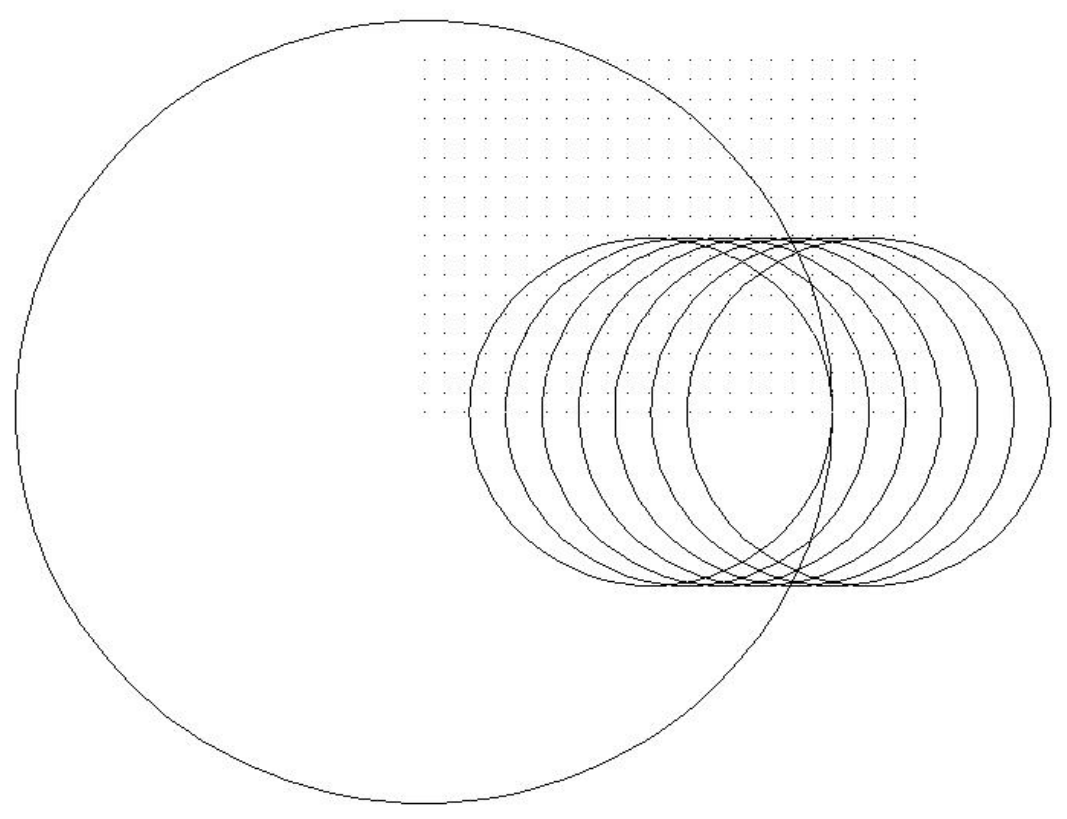

Figure 4. 13 - Traveling beam incident on the lens

The calculated areas were plotted with respect to the center to center distance of the two circles. The larger circle modeled the clear aperture of the lens and the smaller circle modeled the incident light from the laser spark. A polynomial fit of the areas of the eclipsed smaller circle with respect to the center to center distance was used to normalize the dynamic LIBS data.

$$
\phi(x)=-0.0014 x^{3}+0.0223 x^{2}+0.0226 x+0.0012 \ldots
$$

Equation (4-2) best fit the trend in which the area of the eclipsed smaller beam decreased as the center to center distance of the two circles increased. Where $\phi(x)$ is the area of the beam being eclipsed and $\mathrm{x}$ represents the center to center distance of the two circles. The end segment of the signal, $\mathrm{S}\left(\mathrm{x}_{\mathrm{i}}\right)$, from the dynamic LIBS test can be described as the time dependent behavior, $\beta\left(\mathrm{x}_{\mathrm{i}}\right)$ convoluted with the eclipsing function $\phi\left(\mathrm{x}_{\mathrm{i}}\right)$.

$$
\mathrm{S}\left(\mathrm{x}_{\mathrm{i}}\right)=\phi\left(\mathrm{x}_{\mathrm{i}}\right) \times \beta\left(\mathrm{x}_{\mathrm{i}}\right) \ldots(4-3)
$$


To recover $\beta\left(\mathrm{x}_{\mathrm{i}}\right)$, the normalized eclipsing function, $\phi\left(\mathrm{x}_{\mathrm{i}}\right)$ would be divided into the original signal.

$$
\beta\left(x_{i}\right)=S\left(x_{i}\right) \div \phi\left(x_{i}\right) \ldots(4-4)
$$

The noise in the CCD response was magnified as $\phi(x)$ approached zero. Any trends in temporal behavior were recognized and forecasted through the magnified noise levels.

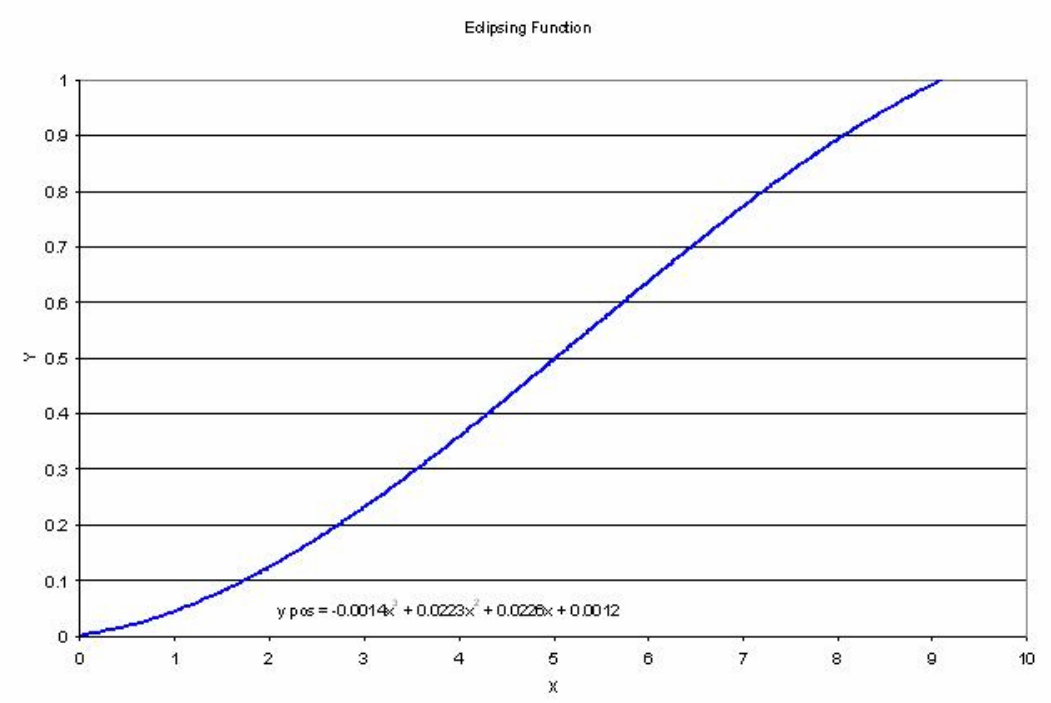

Figure 4. 14 - Eclipsing Function

Dynamic LIBS shows time-dependent characteristics of multiple emission lines within a single laser spark. Complications arise in signal analysis due to experimental conditions. The scanning mirror was not synchronized with the rest of the experimental set up. The randomness of the testing procedure resulted in a hit or miss testing method and made reproducibility of the dynamic data impossible. The separation of the optics from the spectrograph produced spatial instability. Each dynamic LIBS shot had a unique eclipsing range.

The application of a time scale and de-convolution of the eclipsing effect of the later times followed the initial background correction. Emission lines were plotted together to observe their temporal behavior simultaneously. 
Emission Llines Mix 9 shot 11

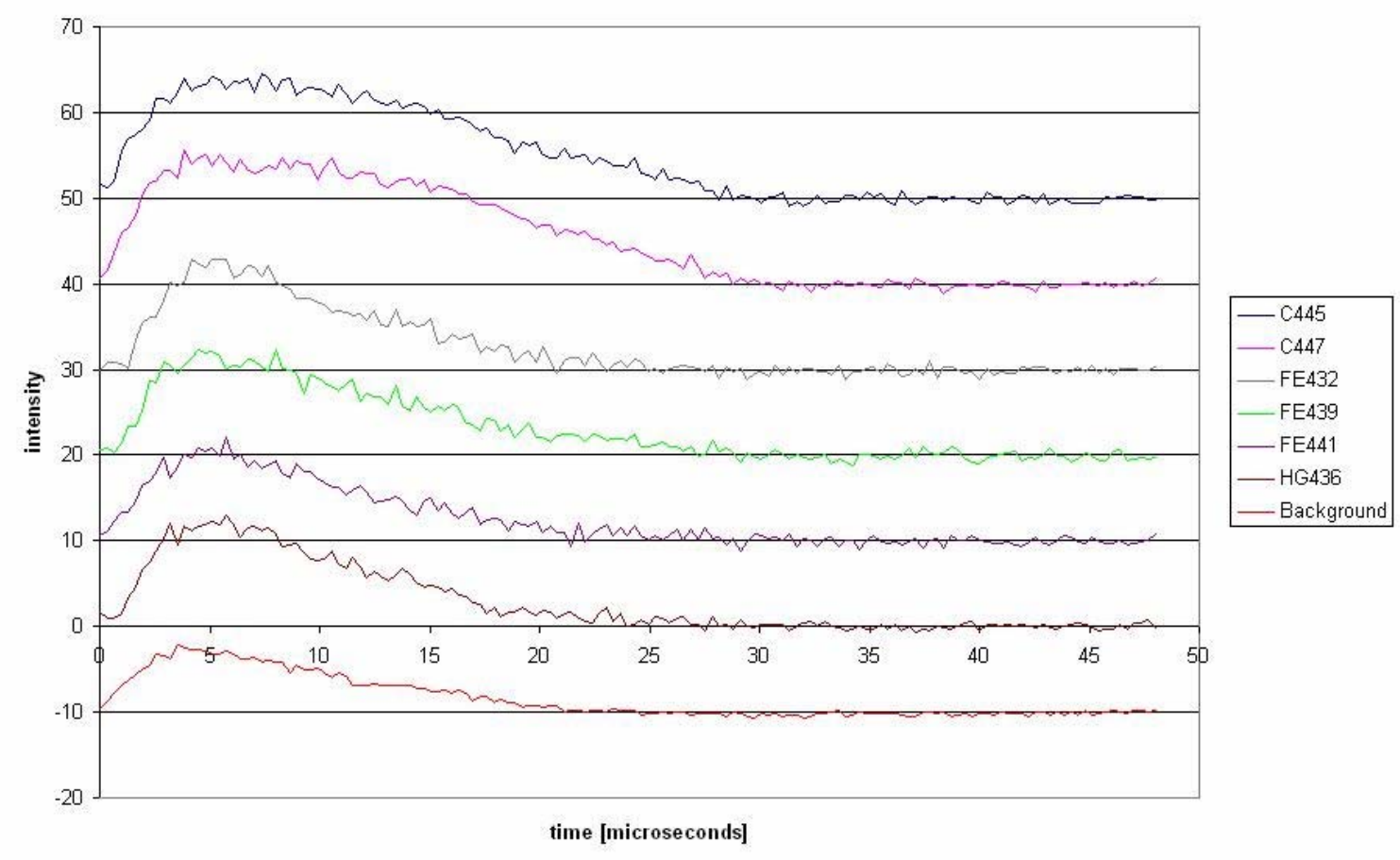

Figure 4. 15 - Convoluted Emission Line Data separated by 10 counts for clarity

The later eclipsing effect, which takes place between ten and fifteen microseconds after spark formation, makes the remaining signal appear linear. The beginning time $(\mathrm{t}=$ 0) shows convolved data from both eclipsing of the final focusing lens, and from the pinhole of the spatial filter. The carbon related 445 and $447 \mathrm{~nm}$ lines show a "plateau" before being eclipsed by the focusing lens. Iron and mercury do not show this plateau. The rise times observed by the spark are convolved with the pinhole limitation and eclipsing. The data at the later times in the spark can be recovered by normalization of the eclipsing function, $\phi\left(\mathrm{x}_{\mathrm{i}}\right)$, where $\mathrm{x}_{\mathrm{i}}$ represents the segment of data affected by eclipsing. 


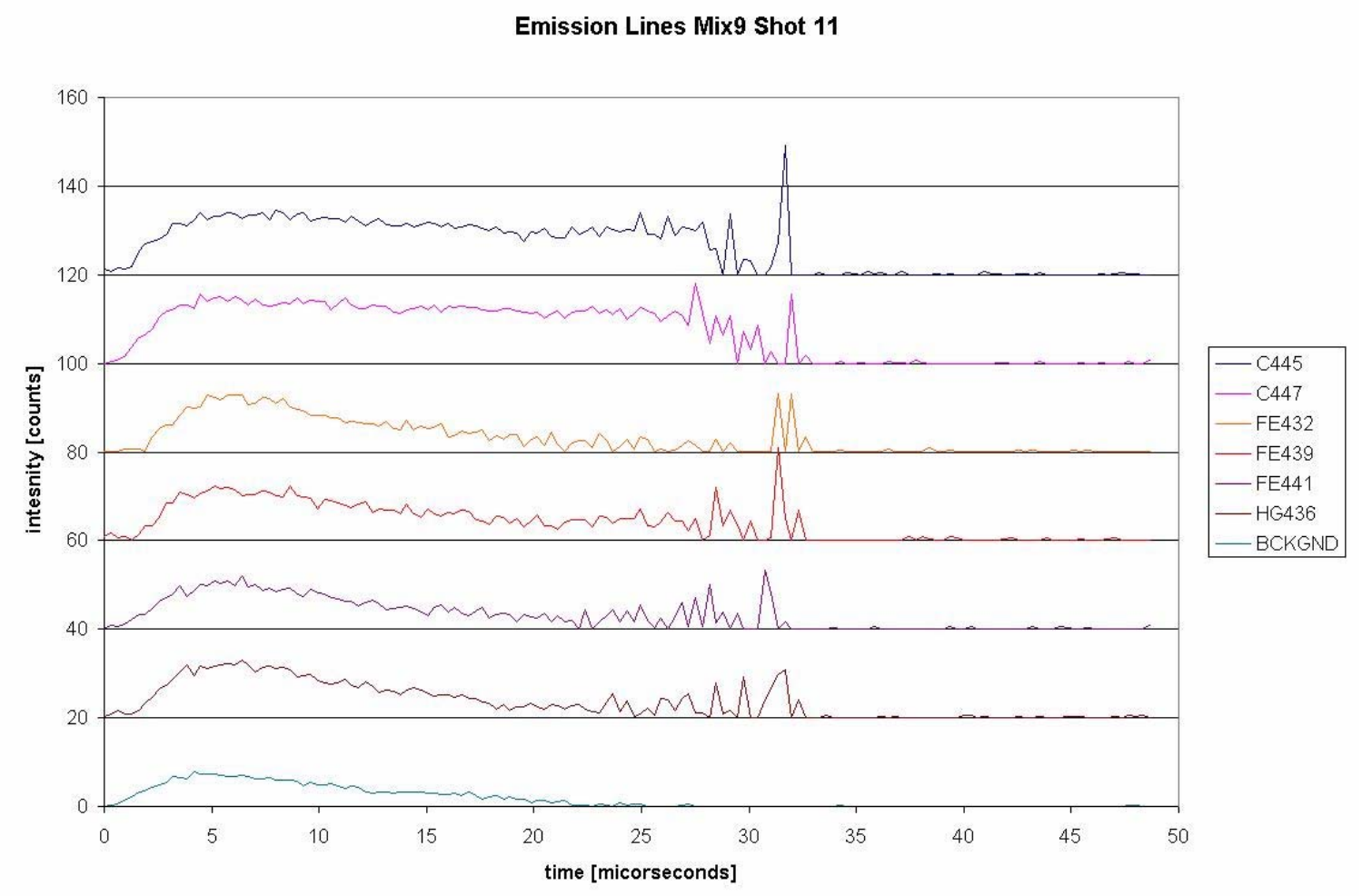

Figure 4. 16 - Emission Lines with eclipsing correction at later spark life separated by 20 counts for clarity

Background

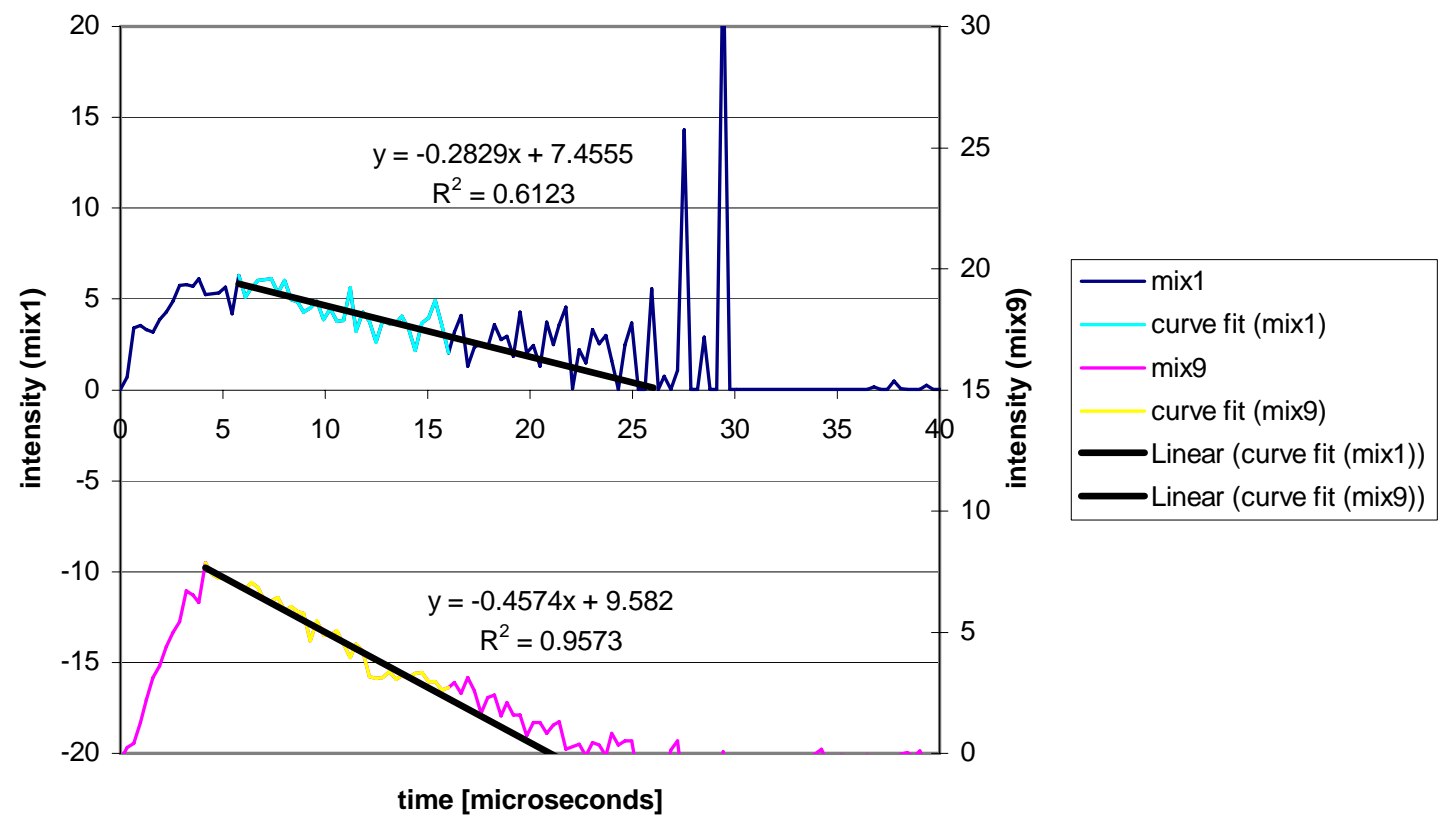

Figure 4. 17 - Background Radiation Decay 
The background decay represents the continuum emission and is temperature driven. It shows linear decay. The time dependent signals from the background emission can be used to observe time-temperature characteristics within the plasma.

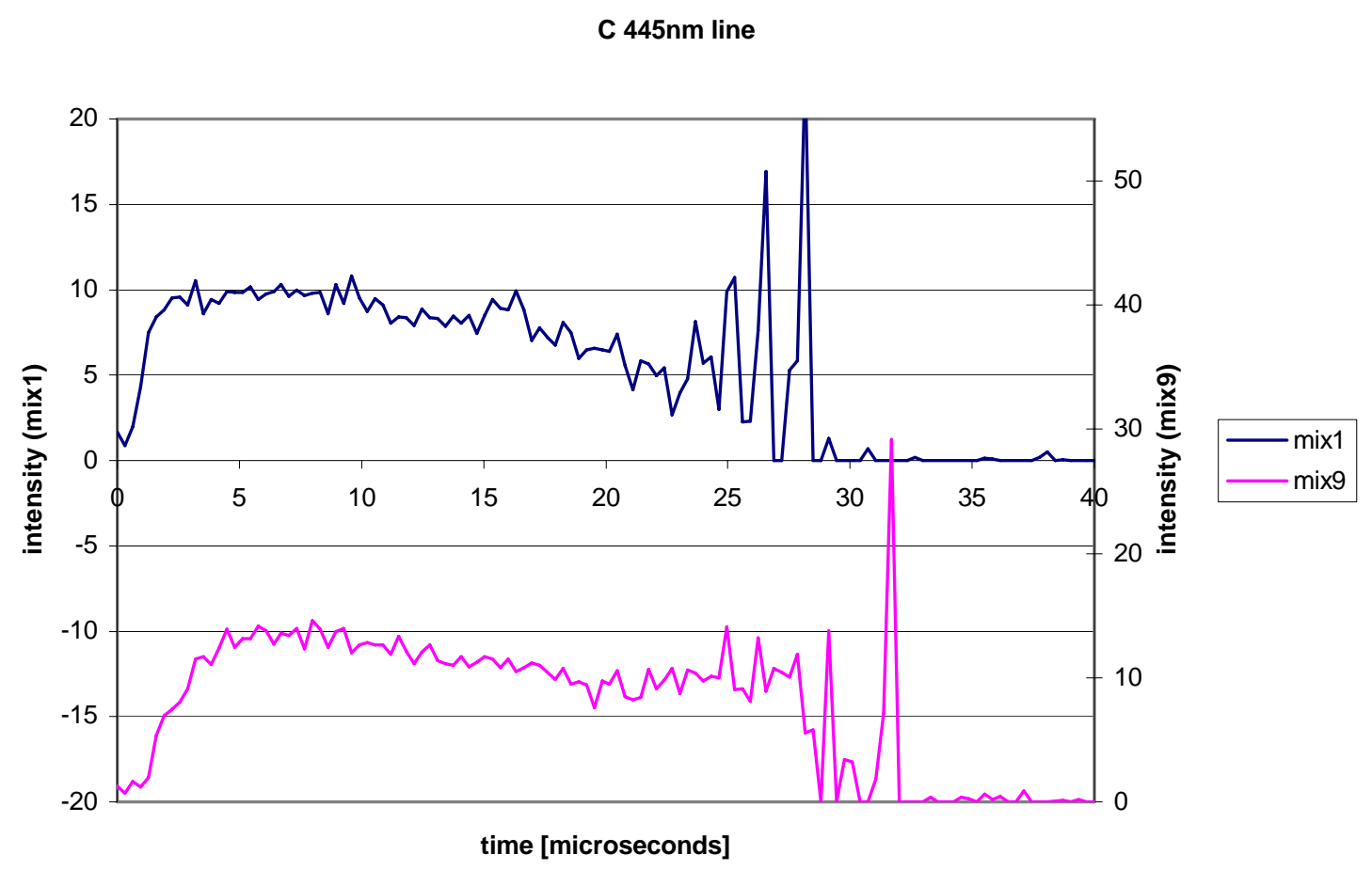

Figure 4. 18 - C 445nm temporal behavior

Carbon related emission lines show discontinuity upon observation. There is no observed trend of decay. The temporal data of the carbon related emission lines show different qualities to those of iron and mercury. The discontinuity in the carbon related emission lines could be due to effects such as re absorption or interaction with another element. 
C $447 \mathrm{~nm}$ line

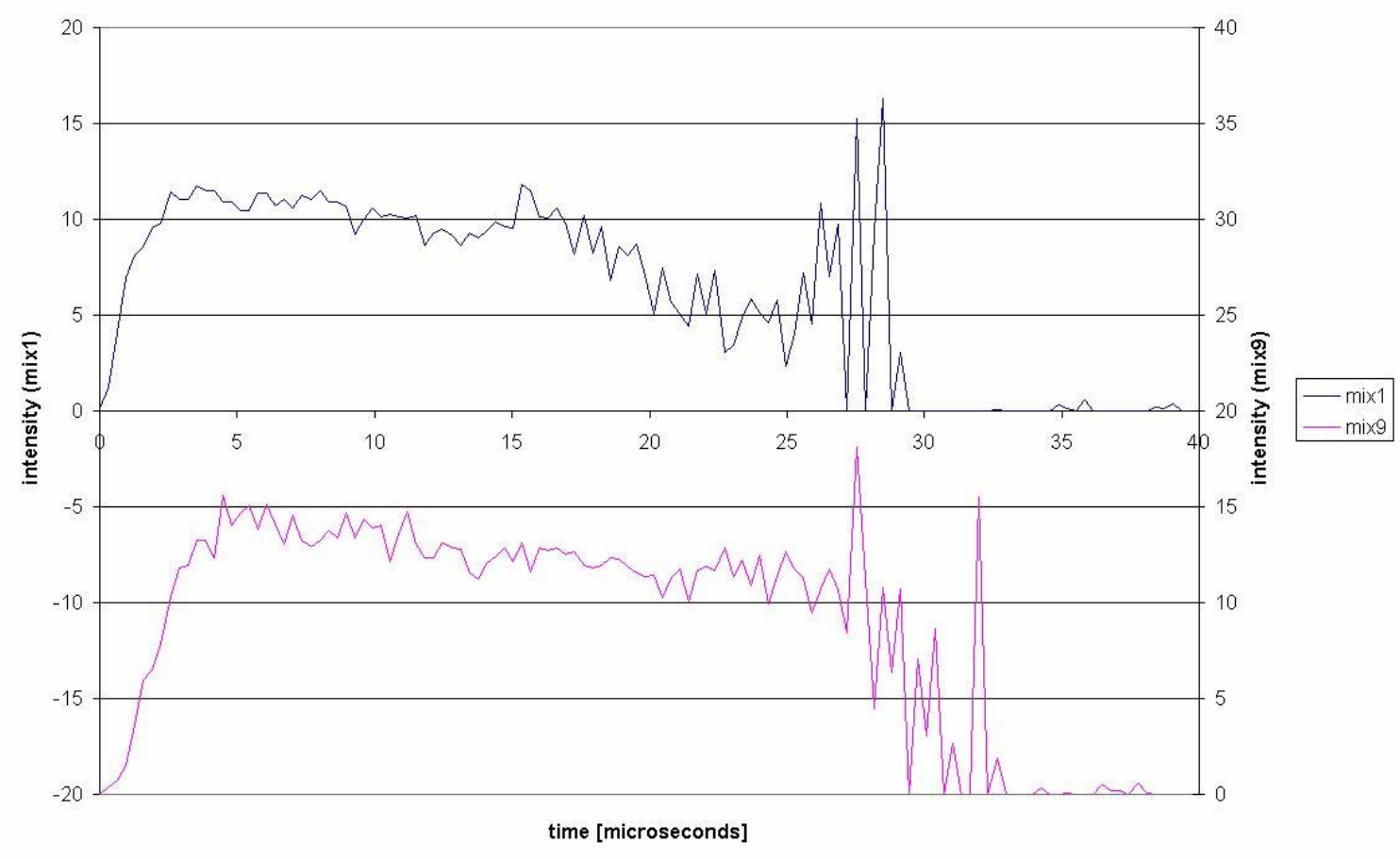

Figure 4. 19 C 447nm temporal behavior 


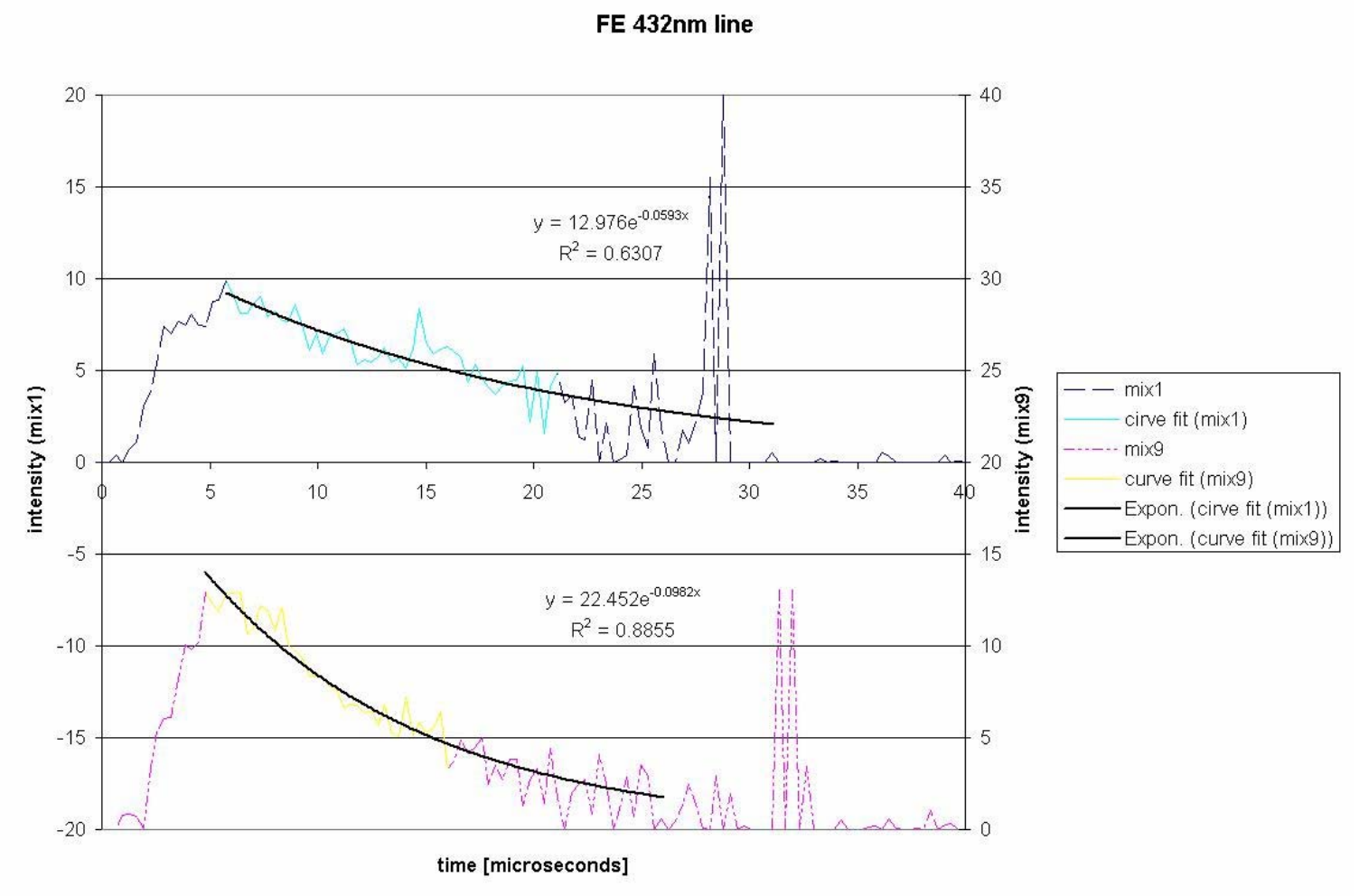

Figure 4. 20 - Fe 432nm temporal behavior 


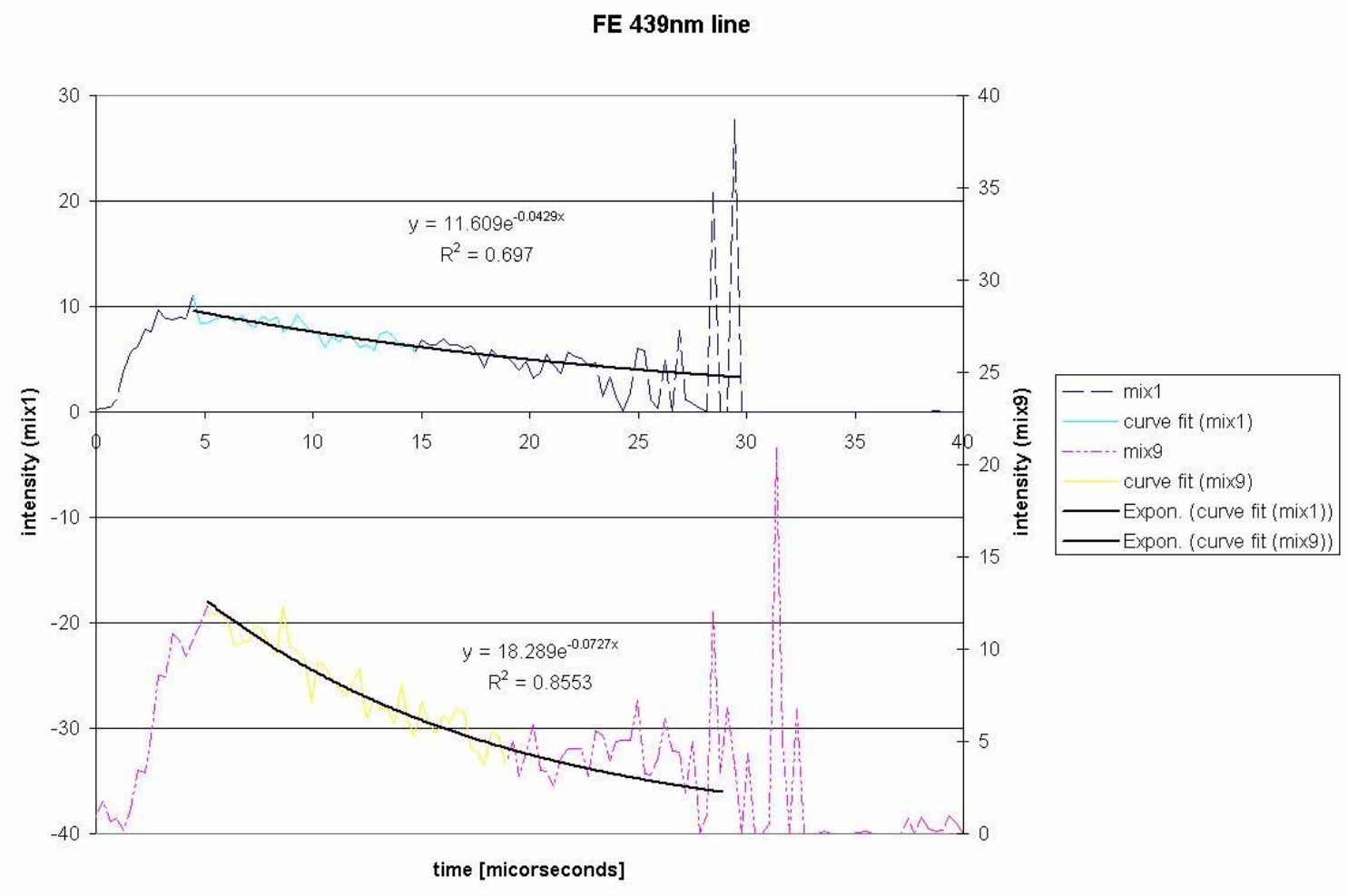

Figure 4. 21 - Fe 439nm temporal behavior 


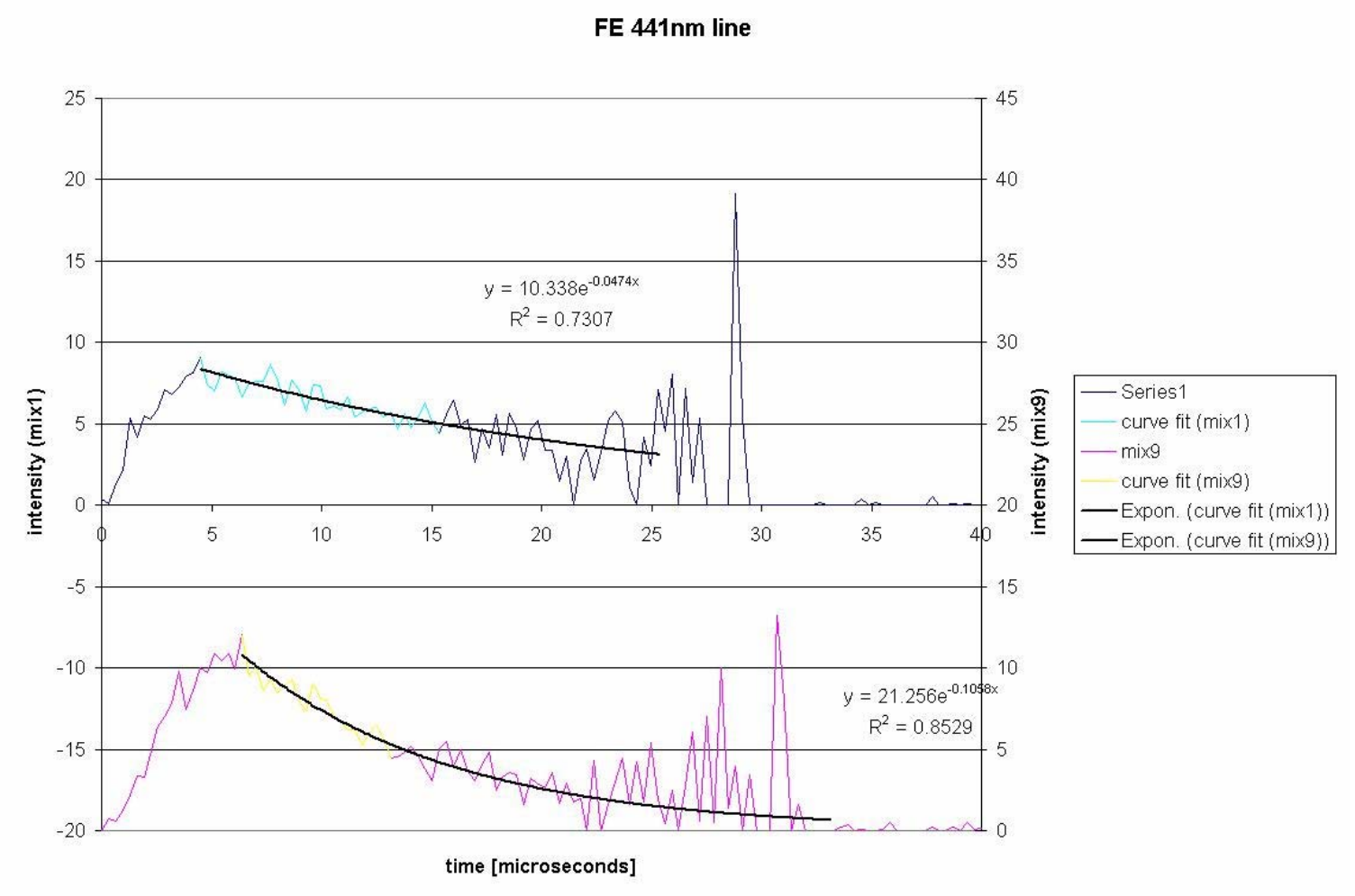

Figure 4. 22 - Fe 441nm temporal behavior 


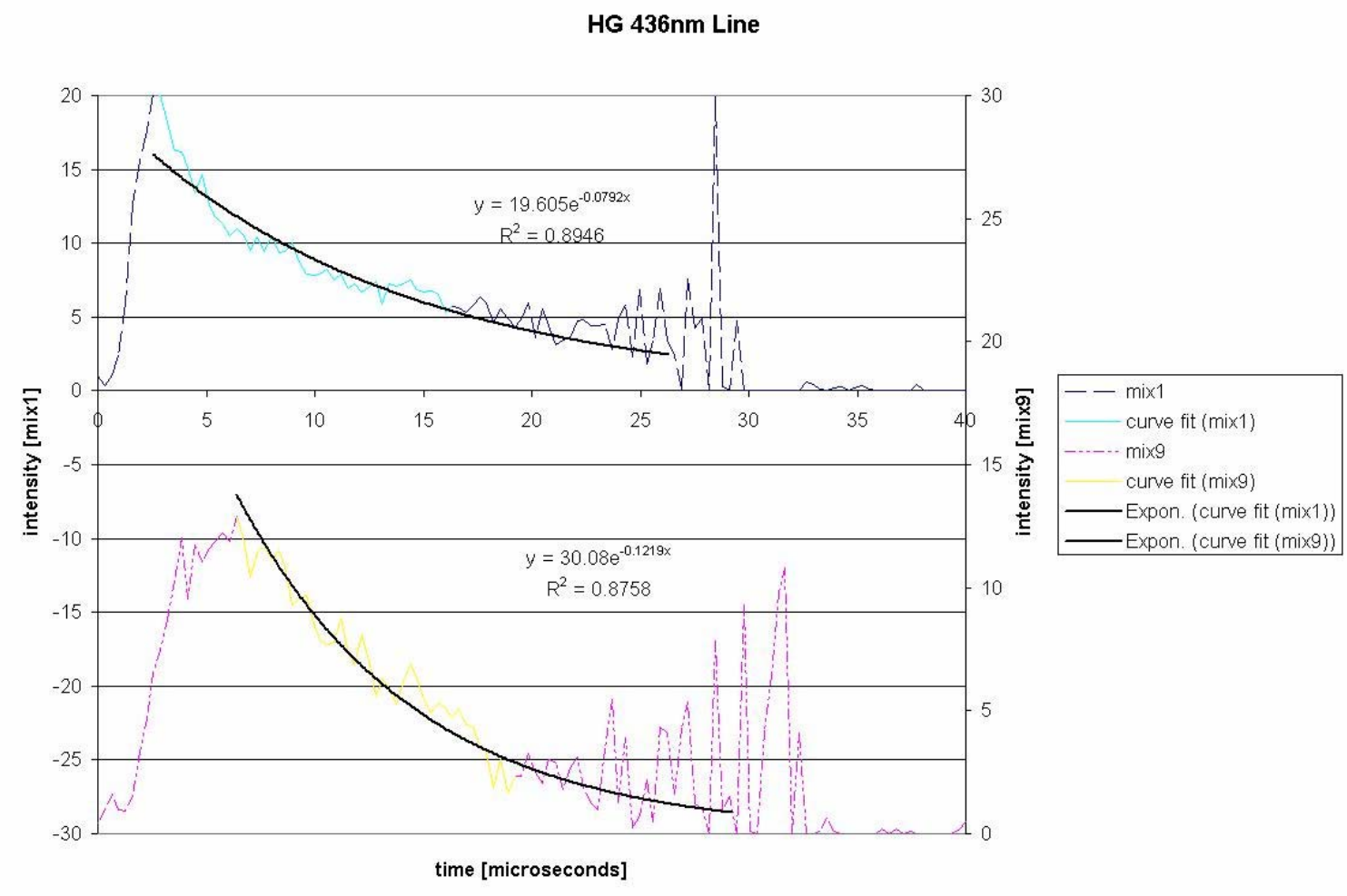

Figure 4. 23 - Hg 436nm temporal behavior

The time dependent signals from both iron and mercury show exponential decay. Each emission line is observed to display different decay rates. Mercury shows a faster decay signal than iron. The data obtained from the dynamic LIBS signal shows a potential of comparing decay rates of different elements and obtaining the half lives of each emission line, displaying uniqueness of the temporal characteristics of each emission line. 

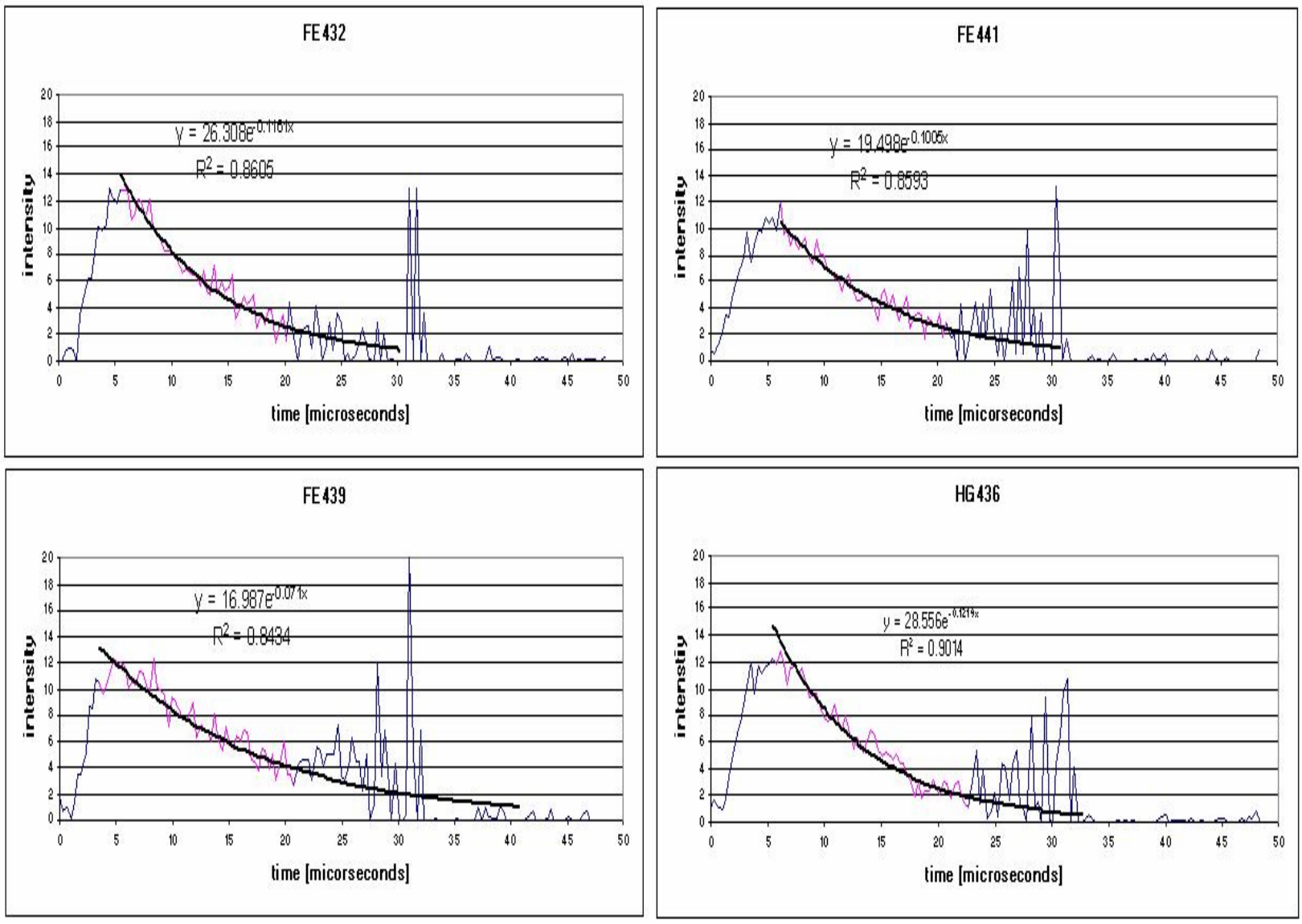

Figure 4. 24 - Comparison of Iron and Mercury Lines in Mix9 


\section{CHAPTER 5: CONCLUSIONS AND RECOMMENDATIONS}

\subsection{Conclusions}

A LIBS system has been developed to detect C, Fe, and Hg in coal. Significant information has been obtained in analyzing the signals. The ability to detect C, Fe, S, and Hg by this system has produced mixed results. Upon obtaining data, the strength of the modified intensity signal from the collected light was plotted with respect to known concentration in the specimen. The graph of relative intensity with respect to concentration resulted in a strong correlation within the carbon and mercury emission lines.

Carbon, which ranged from 61 - 81\% mass within the synthetic coal mixture, resulted in a linear relation between line intensities and signal strength. Carbon related emission lines do not show strong correlation to concentration for analytical purposes. Further testing of the emission lines can verify the use of these lines for analytical purposes.

Use of the 436nm emission line as a means of detecting mercury was accurate to 800ppm. A plot between the signal strength and the concentration yielded a strong correlation, with only one discrepancy point, possibly due to a mixing error. The 800ppm limitation was due to the system hardware. A more sensitive detection instrument is required to attain lower detection limits. Mercury emission line signals at other wavelengths seem to have been quenched by the oxygen in standard atmospheric conditions. The 436nm mercury line was observed in standard atmospheric conditions.

Sulfur could not be detected using this experimental apparatus. This may be due the air quenching effect reported earlier [17]. Since the most sensitive emission lines in 
the sulfur spectrum are below 200nm, a combination of special optics and a vacuum environment are required to optimize the detection of sulfur.

Iron emission lines showed poor correlation between signal strength and concentration. This could be due to the mixing of pure iron in powder form within the synthetic mixtures as opposed to iron oxide. It is hypothesized that during the grinding and mixing process, the iron was drawn together instead of dispersed evenly, creating pockets of highly concentrated iron within the samples.

The analysis of dynamic LIBS data provided basic information on temporal characteristics for individual emission lines. Mercury seems to have a faster decay than iron. Iron and mercury lines show a similar decay characteristic, both differing from carbon. The time dependent signal in the carbon related lines showed discontinuity. The discontinuity of carbon could be due to the effects of re-absorption or interaction with other elements within the plasma. The data upon observation of emission lines of one line compared to another suggests that emission lines posses differing temporal characteristics from one another. The non-synchronization of the scanning mirror to the pulsed laser and CCD controller affected the reproducibility of the dynamic data. The beginning and ends of the dynamic data were convolved with eclipsing and with the limitations of the pinhole of the spatial filter. Data from dynamic LIBS shows potential for determining unique characteristics of each emission line. 


\subsection{Recommendations for Future Work}

\subsubsection{System Improvements}

The sensitivity and accuracy of the LIBS method developed within the scope of this research can be improved upon. This research has shown that all of the elements of interest have the possibility of being quantitatively analyzed much faster than the traditional ASTM methods. Because of the limited detection of mercury and sulfur within the synthetic coal mixtures, future work should be focused on the development of the sensitivity limits and the synchronization of the current LIBS system.

The fine alignment of optics within the experimental LIBS set up could be improved by adding micrometer translation stages for each optical element. Fine alignment within the apparatus could be used to obtain more signal from the image of the LIBS spark. The optical collection system and the spectrograph were not integrated onto a single platform, making the measurements sensitive to external movement. Integration of the spectrograph with the collection optics on a single platform would improve the robustness of the LIBS system. Synchronization of the scanning mirror with the laser and the CCD controller would allow for reproducible dynamic data.

Because of the effects of oxygen quenching mercury and sulfur emission lines, a vacuum environment or an inert gas environment could be created to enhance LIBS signals. The CCD detector could be replaced by an Intensified CCD (ICCD) camera to further enhance the signal. An ICCD camera with time gating capabilities can be up to one thousand times more sensitive than a regular CCD camera. If the ICCD camera is used as a detector, and the samples are placed in a vacuum environment, the current LIBS apparatus could attain greater sensitivity to emission lines. 
The 436nm mercury line was observed in atmospheric conditions. With the use of an ICCD camera coupled with fine optical alignment capability, the 436nm line could be used to detect mercury using LIBS without a vacuum or inert gas environment.

To improve upon the correlation of the iron signal, coal can be mixed with iron salts instead of pure iron. The iron salts should not be clumped together during grinding, and should produce a better distribution throughout the mixture. This would result in a greater probability of iron being observed throughout the synthetic mixtures instead of in concentrated areas, giving a more accurate representation of the iron concentration within the coal sample. Improvements within the pellet making procedure in this experiment can result in more accurate measurements and a better mixture for calibration of the LIBS system.

Dynamic LIBS testing gives us qualitative data of how different emission lines behave. The analysis of the dynamic LIBS signals allowed for observations of timedependent characteristics of different emission lines. Because the system was not synchronized, a hit or miss method was relied upon to obtain usable dynamic LIBS signals. Synchronization of the system would define a range of eclipsing on the detector, as well as make reproducible results. Anomalies in the dynamic LIBS data were observed, which could be due to the lack of reproducible results. The final focusing lens can be made bigger to avoid eclipsing effects on the dynamic data. Fine alignment of the optics will produce better signal obtained from the laser spark.

\subsubsection{The Ideal LIBS Apparatus}

With all the recommended system improvements being implemented into one system, a significantly improved LIBS system can become a reality. The apparatus and 
procedure of this ideal apparatus is very similar to this experimental work. Differences between the ideal and current system would be the vacuum chamber or inert gas purge in which LIBS testing can be done. Though the implementation of a vacuum chamber will result in the loss of instrument mobility and on-line measurements, ambient air that quenches sulfur and mercury emissions will be removed, resulting in a detectable sulfur signal and greater sensitivity to mercury. The ideal LIBS apparatus would have a high resolution spectrograph that interfaces with a specialized UV sensitive ICCD to select and tune the wavelength range, a vacuum chamber with specialized optics to tune in on the ultraviolet sulfur emission lines. Synchronization between the laser and the spinning mirror will allow reproducible dynamic LIBS testing results, which show potential for determining uniqueness of individual emission lines observed. With these significant improvements to the system, an ideal LIBS apparatus can be built that can improve upon the existing results obtained in this research. 


\section{REFERENCES}

[1] Yalcin, Crosley, Smith, Faris; "Influence of ambient conditions on the laser air spark”, Applied Physics B (1999) 68(1) 121-130

[2] Barbini, Colao, Fantoni, Lazic, Palucci, Pershin; “ Laser Induced Breakdown Spectroscopy: Progress towards a tool for quantitative elemental analysis of main constituents and trace elements in different condensed matrices”, Journal of Russian Center of Laser Physics 5 (2001) 10.8

[3] Skoog, Holler, Nieman; Principles of Instrumental Analysis, $5^{\text {th }}$ Ed. ; 1998 Harcourt Brace \& Company

[4] Radziemski; "From Laser to LIBS, the path of technology development", Spectrochimica Acta Part B 57 (2002) 1109 -1113

[5] Schobert; Coal: The Energy Source of the Past and Future; American Chemical Society, Washington DC, 1987.

[6] ASTM D2013-04, "Standard Practice of Preparing Coal Samples for Analysis", ASTM International, West Conshohocken, Pa, 2004

[7] ASTM D3178-89(2002), "Standard Test Methods for Carbon and Hydrogen in the Analysis Sample of Coal and Coke”, ASTM International, West Conshohocken, Pa, 2004 
[8] ASTM D3177-02, "Standard Test Methods for Total Sulfur in the Analysis Sample of Coal and Coke”, ASTM International, West Conshohocken, Pa, 2004

[9] ASTM D3174-04, "Standard Test Method for Ash in the Analysis Sample of Coal and Coke from Coal”, ASTM International, West Conshohocken, Pa, 2004

[10] ASTM D3684-01, "Standard Test Method for Total Mercury in Coal by the Oxygen Bomb Combustion/Atomic Absorption Method”, ASTM International, West Conshohocken, Pa, 2004

[11] ASTM D6349-01, "Standard Test Method for Determination of Major and Minor Elements in Coal, Coke, and Solid Residues from Combustion of Coal and Coke by Inductively Coupled Plasma-Atomic Emission Spectrometry”, ASTM International, West Conshohocken, Pa, 2004

[12] Chadwick, Body; "Development and Commercial Evaluation of Laser-Induced Breakdown Spectroscopy Chemical Analysis Technology in the Coal Power Generation Industry”, Applied Spectroscopy (2002), 56(1) 70-74

[13] Kurihara, Ikeda, Izawa, Deguchi, Tarui; “Optimal boiler control through real-time monitoring of unburned carbon in fly ash by laser-induced breakdown spectroscopy”, Applied Optics (2003), 42(30) 6159-6165 
[14] Ottesen, Baxter; "Laser Spark Emission Spectroscopy for in Situ, Real-Time Monitoring of Pulverized Coal Particle Composition”, Energy \& Fuels (1991) 304-312

[15] Wiesburg, De Saro, Craparo; "Real Time, In-Situ Laser Sensor for Feedsock Monitoring in Gasifiers”, Proceedings of the International Technical Conference on Coal Utilization \& Fuel Systems (2004), 29th(Vol. 1) 445-456

[16] Martin, Wullshleger, Garten, Palumbo; “Laser-induced breakdown spectroscopy for the environmental determination of total carbon and nitrogen in soils”, Applied Optics (2003), 42(12) 2072-2077

[17] Sturm, Noll; "Laser-induced breakdown spectroscopy of gas mixtures of air, CO2, N2, and C3H8 for simultaneous C, H, O and N measurement”, Applied Optics (2003), 42(30) 6221-6225

[18] Ferioli, Puzinauskas, Buckley; "Laser-Induced Breakdown Spectroscopy for OnLine Engine Equivalence Ratio Measurements”, Applied Spectroscopy (2003), 57(9) 1183-1189

[19] Sturm, Peter, Noll; “Steel Analysis with Laser-Induced Breakdowns Spectrometry in the Vacuum Ultraviolet”, Applied Spectroscopy (2000), 54(9) 1275-1278 
[20] Dudragne, Adam, Amouroux; "Time-resolved laser-induced breakdown spectroscopy: Application for qualitative and quantitative detection of $\mathrm{F}, \mathrm{Cl}, \mathrm{S}$, and $\mathrm{C}$ in air”, Applied Spectroscopy (1998), 52(10), 1321-1327

[21] Harrison; Massachusetts Institute of Technology Wavelength Tables, 1969 M.I.T Press, Cambridge MA

[22] Vander Wal, Ticich, West, Householder; "Trace metal detection by laser-induced breakdown spectroscopy”, Applied Spectroscopy (1999), 53(10), 1226-1236

[23] Lazzari, De Rosa, Rastelli, Ciucci, Palleschi, Salvetti; "Detection of mercury in air by time-resolved laser-induced breakdown spectroscopy technique”, Laser and Particle Beams (1994), 12(3), 525-530

[24] Gleason, Hahn; "The effects of oxygen on the detection of mercury using laserinduced breakdown spectroscopy”, Spectrochimica Acta Part B (2001), 56, 419-430

[25] Cheng; "Real-time measurement of trace metals on fine particles by laser-induced plasma techniques”, Fuel Processing Technology (2000), 65-66, 219-229

[26] Tognoni, Palleschi, Corsi, Cristoforetti; "Quantitative micro-analysis by laserinduced breakdown spectroscopy: a review of the experimental approaches", Spectrochimica Acta Part B (2002), 57, 1115-1130 


\section{APPENDIX A: FILE PROCESSING}

This appendix is a walkthrough of the file processing procedure used throughout this research to process the static LIBS data. This was done for each individual static shot for each synthetic mixture.

\section{Step 1: Open the file in Winspec32}

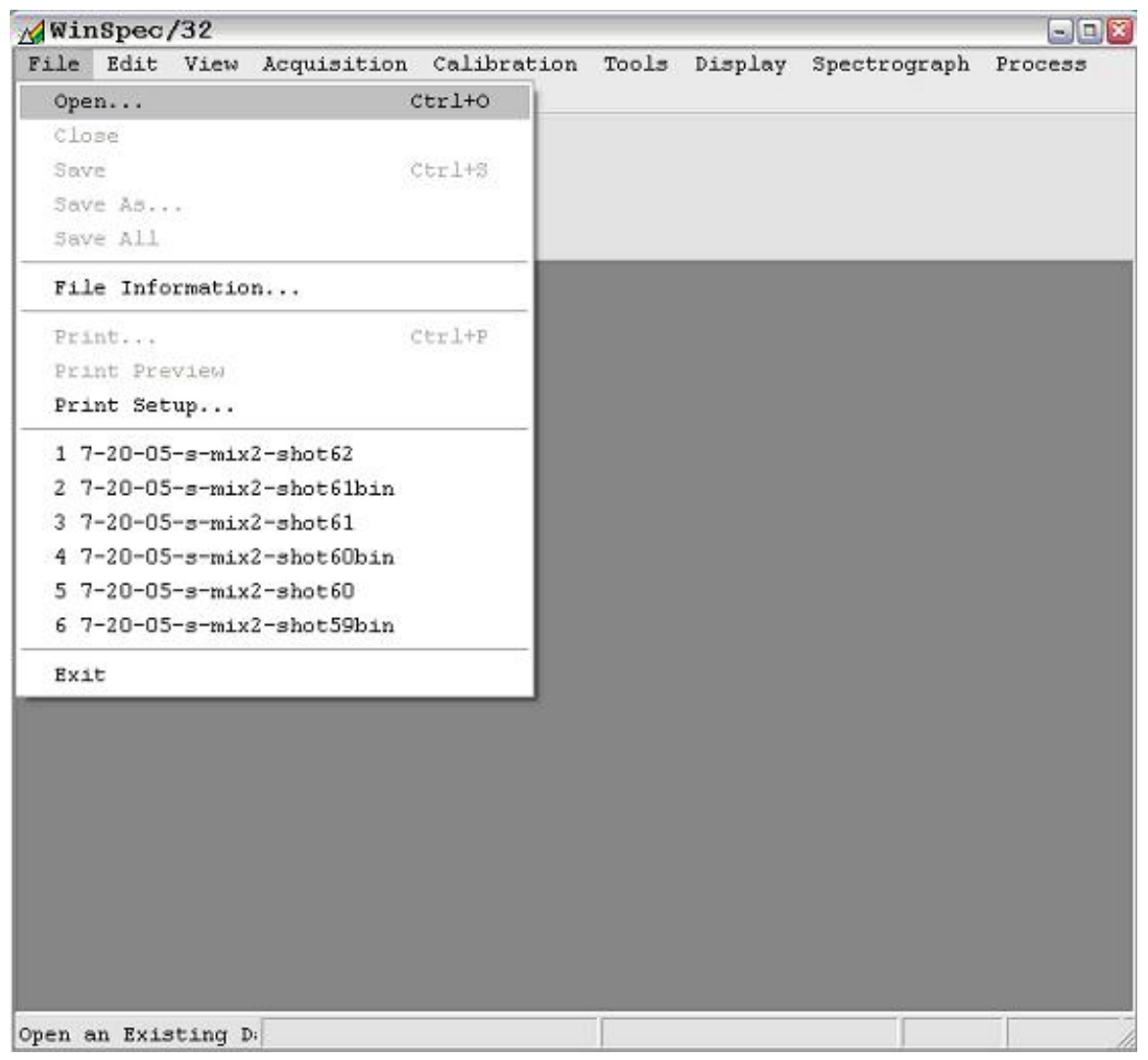

Figure A. 1 - Files opened in Winspec32 


\section{Step 2: Binning the file}

Binning is a process that will take the sum of all the pixels of a single axis. When the images are binned, they are binned about the y-axis because the spectral line positioning is defined on the x-axis. Without binning, quantitative analysis would be more difficult because binning improves the signal to noise ratio within the data.

Go to Process/Binning and Skipping

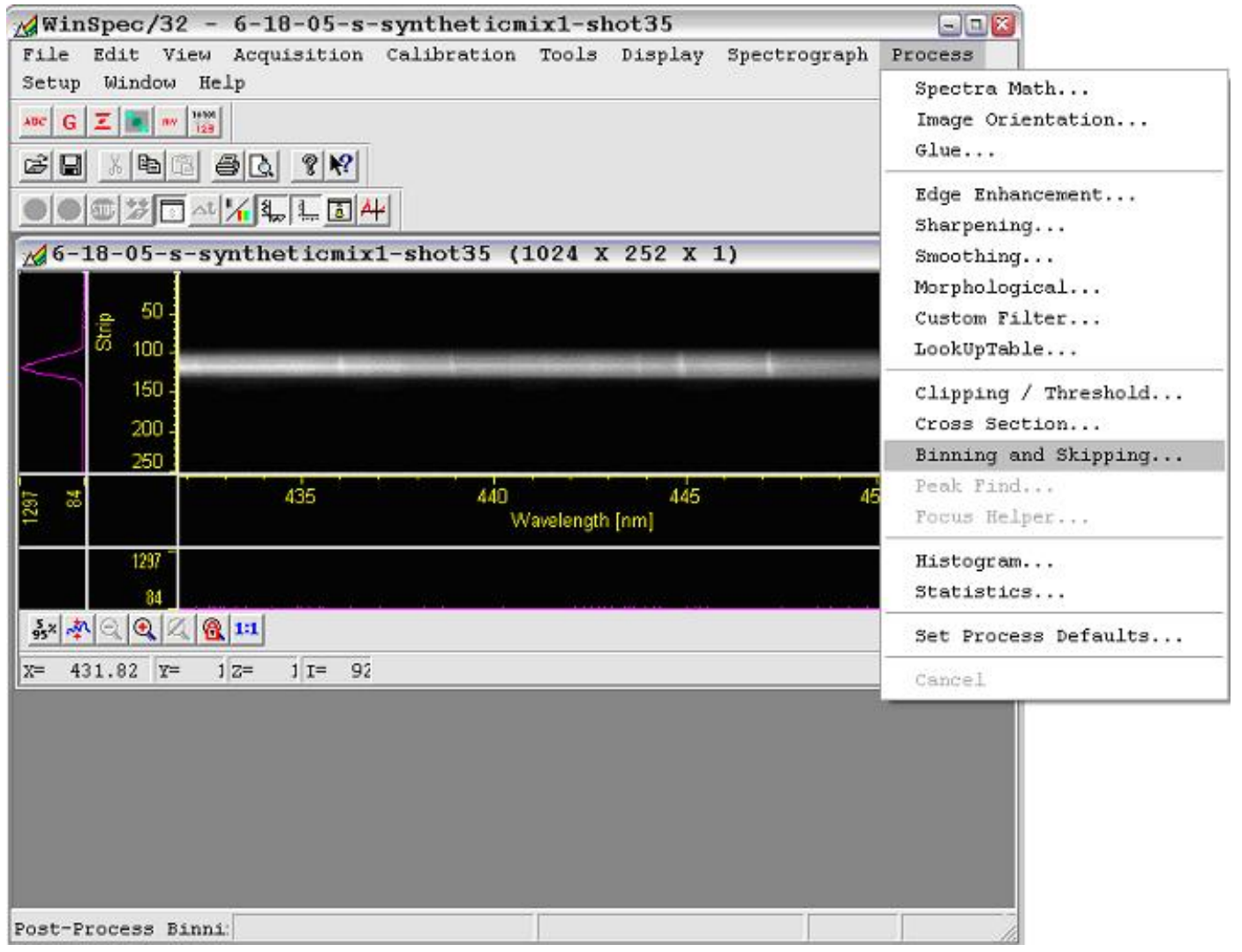

Figure A. 2 - Binning and Skipping 
Select the filename to be binned

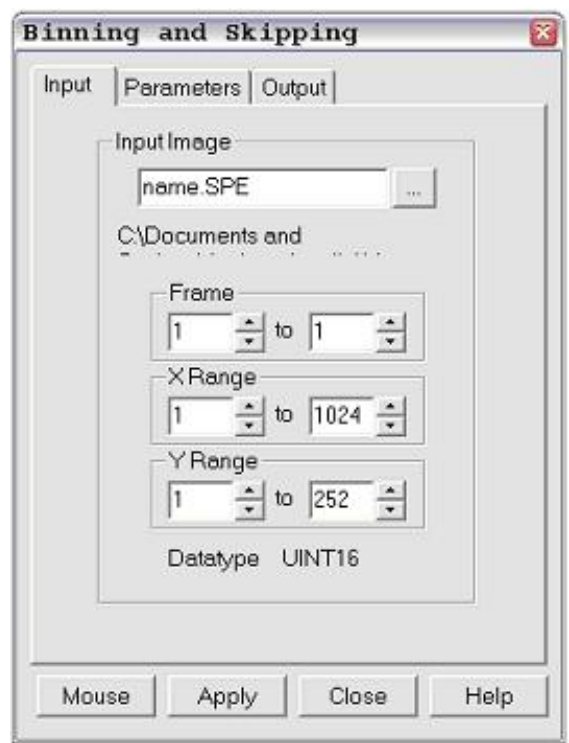

Figure A. 3 - input filename

Select the parameters.

Notice that since there is no skipping involved, the skipping parameters are set at zero, and since a binning of the y-axis is desired, the y dimension goes to 252. The xaxis stays at 1 because it is not being binned. The signal from the static shot is very strong. Both a full-frame binning and region of interest binning were compared using a 10 shot average from synthetic mix 1 shot 1 . The difference in the signal to noise ratio was observed to be negligible and not time-efficient. 


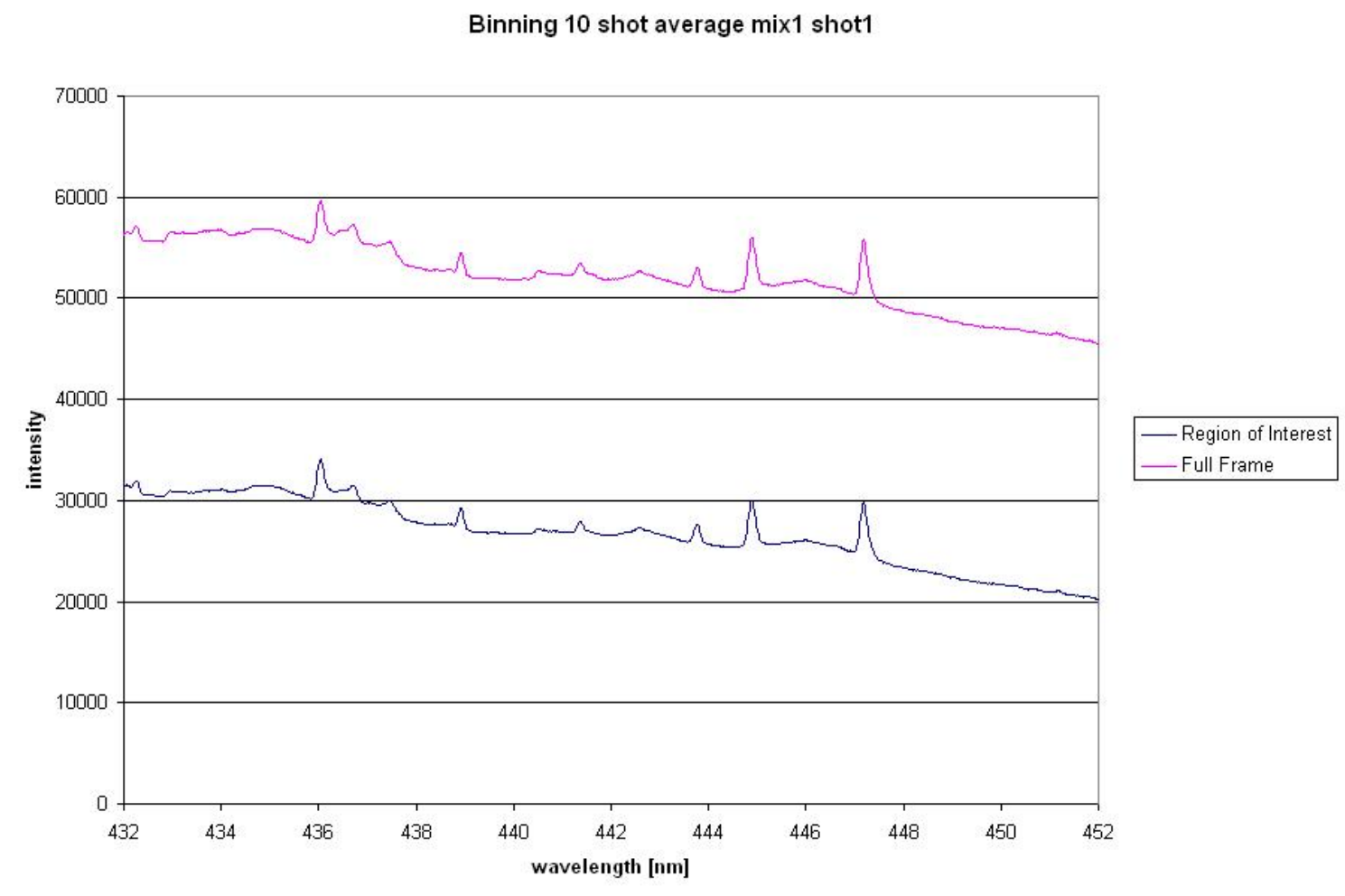

Figure A. 4 - ROI vs. Full Frame Binning

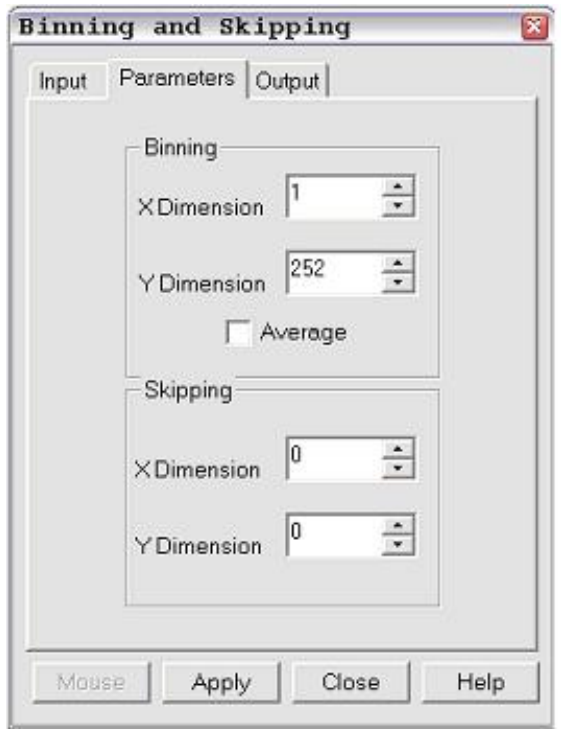

Figure A. 5 - selection of parameters 
Select the output filename

Winspec32 automatically defaults to either the current input filename or "untitled". To avoid saving over files, the notation "filename-bin" is used. For example "7-20-05-s-sampleA-shot1" is the filename, for the first shot of sample A. The binned filename would be “7-20-05-s-sampleA-shot1-bin”

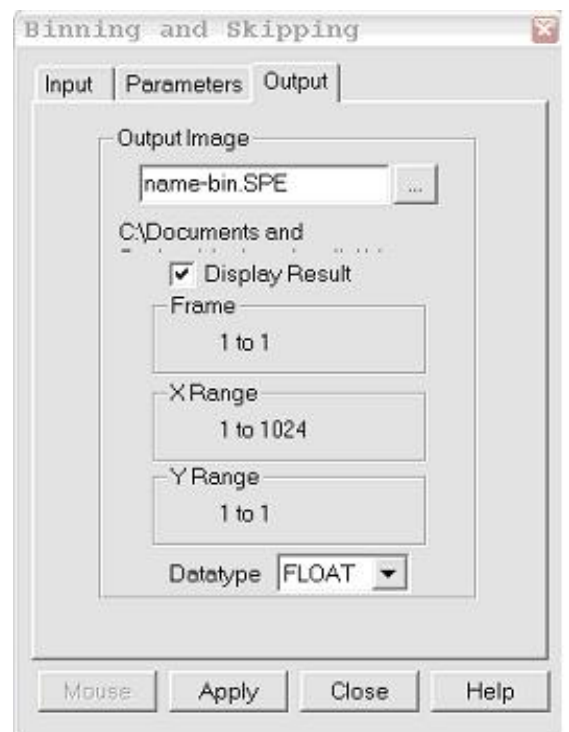

Figure A. 6 - selection of output filename

After all of these steps are followed, click the "Apply” button and Winspec32 will bin the file. Binned files look like the spectra seen in published papers and journals, having a y-axis representing the intensity of the line, and the $\mathrm{x}$-axis representing the wavelength of the spectral range. 


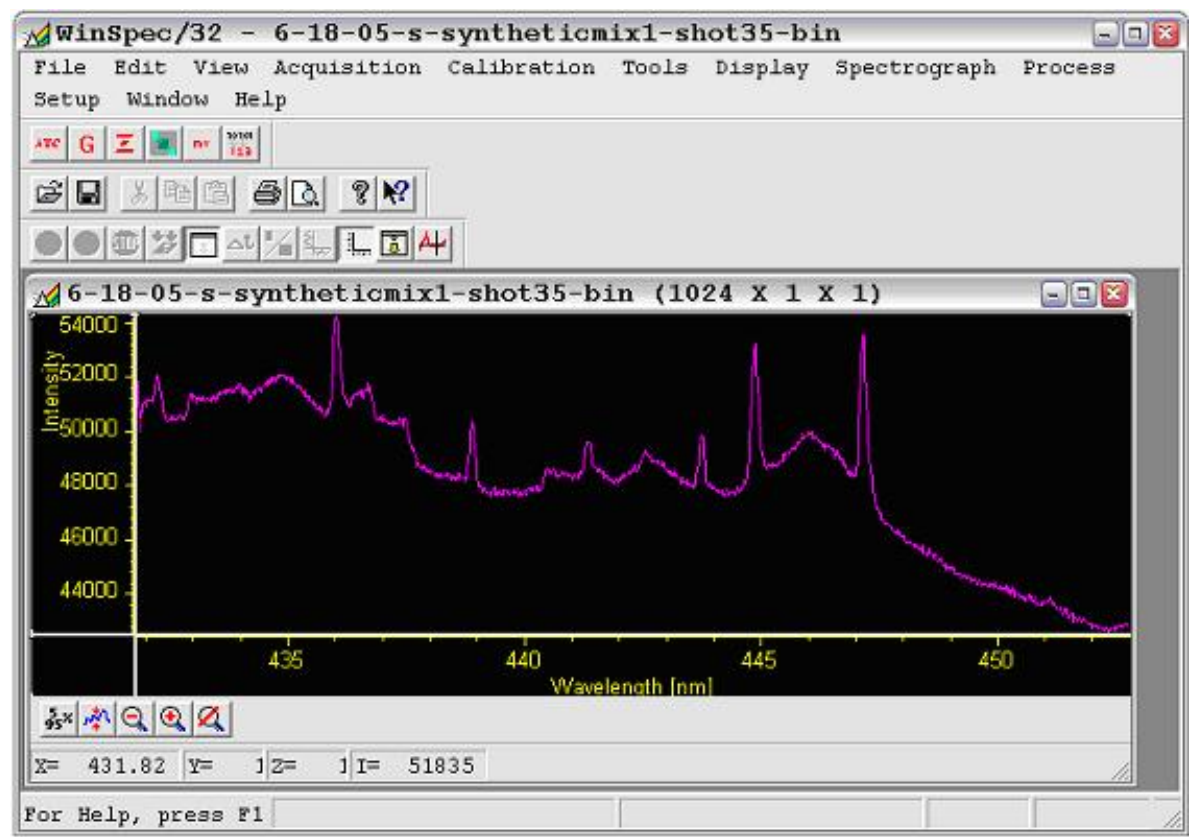

Figure A. 7 - a binned file in Winspec32

\section{Step 3: Conversion to Grams/AI}

Before this operation is done, make sure that both Grams/AI and Winspec32 are both open and running. Hit the zap-grams button in Winspec32 (circled).

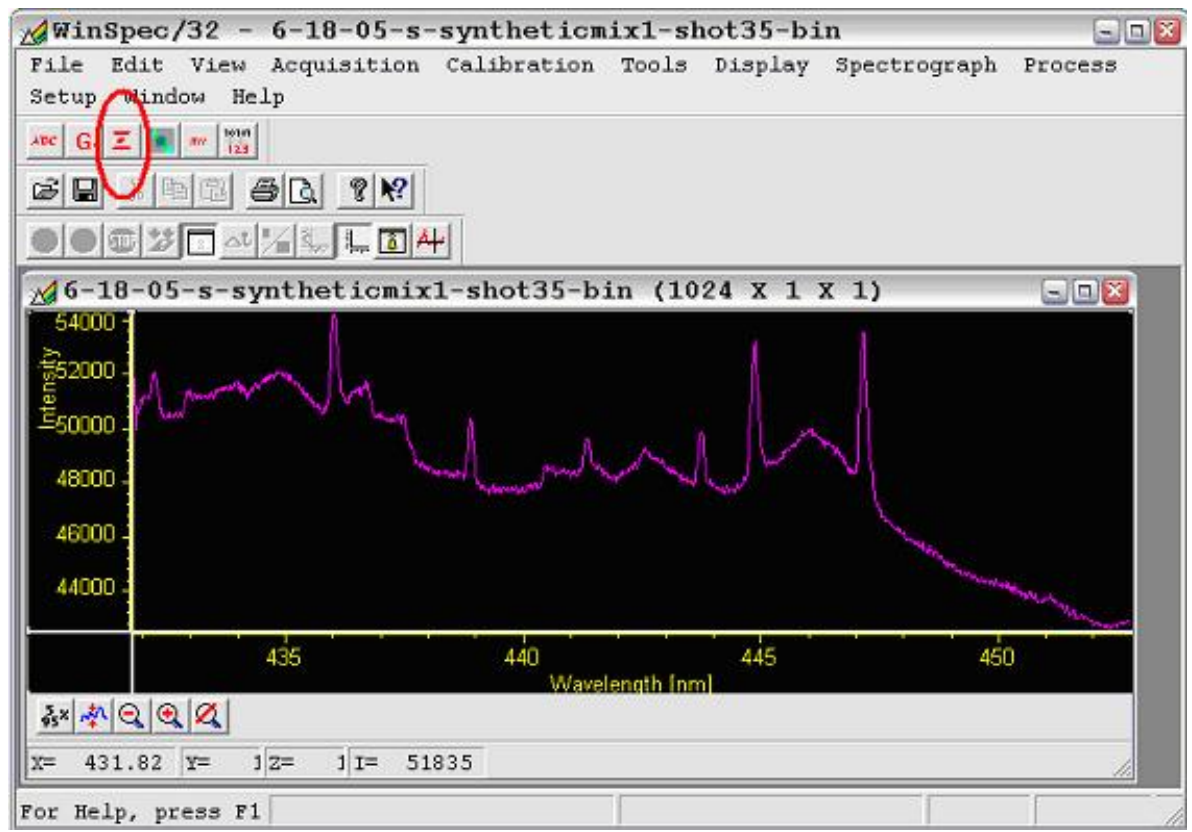

Figure A. 8 - the Zap-Grams button 
Here is what the figure will look like in Grams/AI.

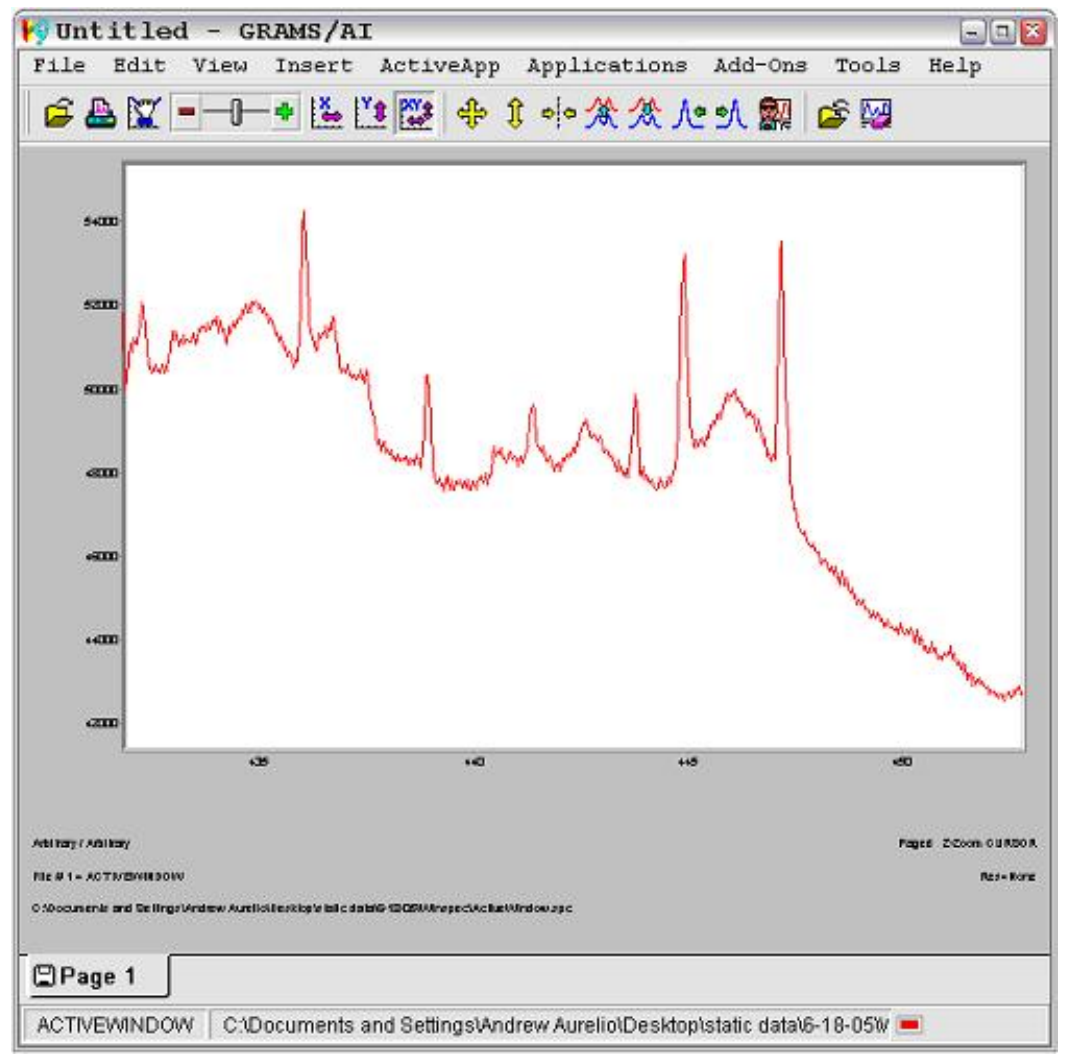

Figure A. 9 - Grams/AI Window

\section{Step 4: Conversion of Grams/AI to Microsoft Excel}

After the data is binned and put into Grams/AI, it is converted into excel. Excel was chosen for its familiarity to all end users and ease to process data. In the Grams/AI window, select the "ActiveApp" option, and go to excel exchange. Once excel exchange is opened, it will give you the option to import Excel files into Grams/AI, or export Grams/AI files to Microsoft Excel. Click on the option to export. After the option chosen, Grams/AI will ask for an active window. It is of most importance that the file path is specified to the appropriate active window. In this experiment, each set of data files were saved into a folder with the appropriate date and sample number. For example, if Sample A was tested on July $20^{\text {th }} 2005$, then the path would be 
C:/mydocuments/LIBS2005/7-20-05/SampleA/activewindow.spc. Active windows can show up in any folder in which you created a Grams/AI file.

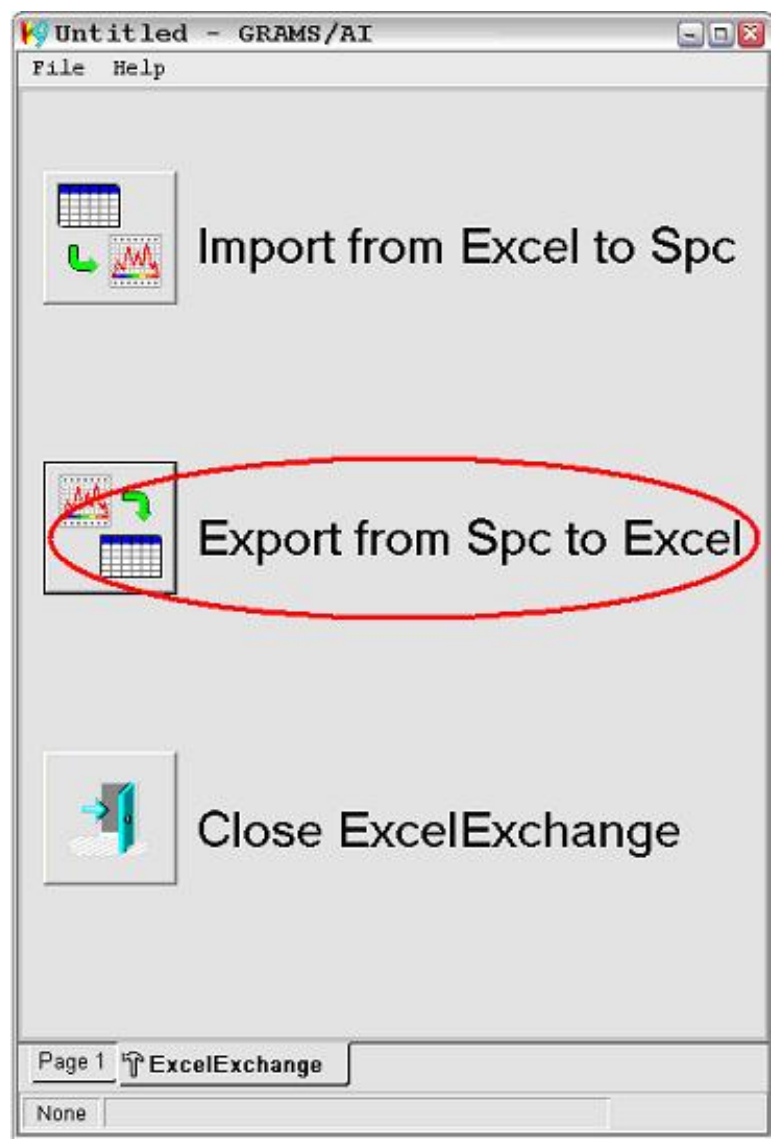

Figure A. 10 - Excel Exchange

After selecting the active window, Grams/AI will prompt for a worksheet name. The name chosen in this experiment was "data". Grams/AI will then prompt the user to select a workbook name. The name used in this research was the sample number followed by the shot number delimited by a hyphen. So in our example, the first shot of Sample A converted to excel would be named A-1, the second shot A-2, and so on and so forth until all shots from sample A are binned, and converted into excel files. 


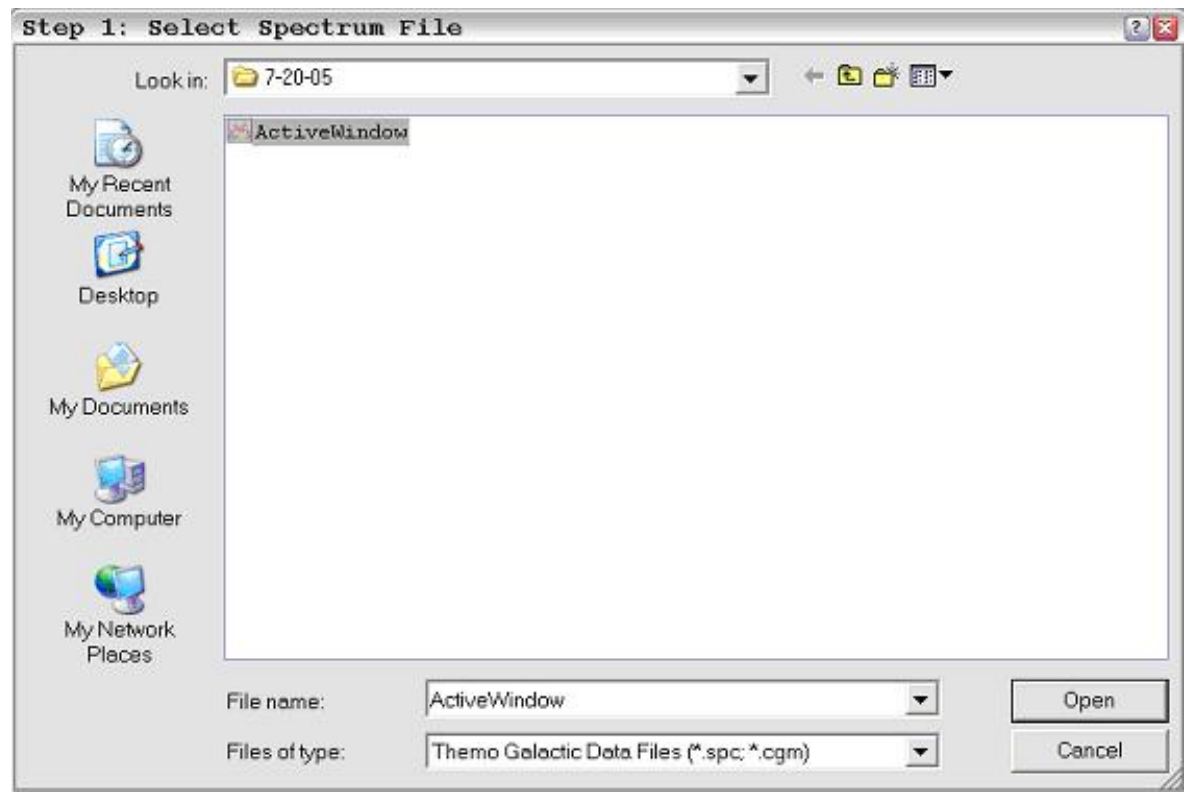

Figure A. 11 - Excel Exchange: Step 1

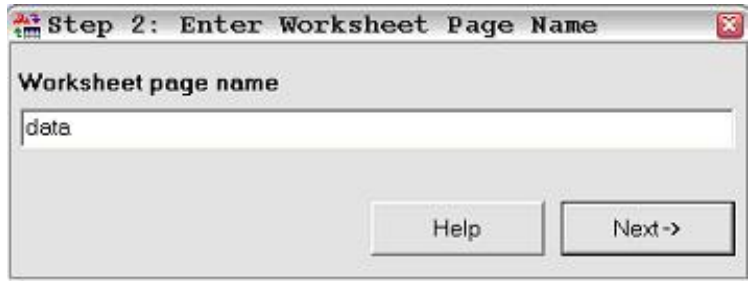

Figure A. 12 - Excel Exchange: Step 2

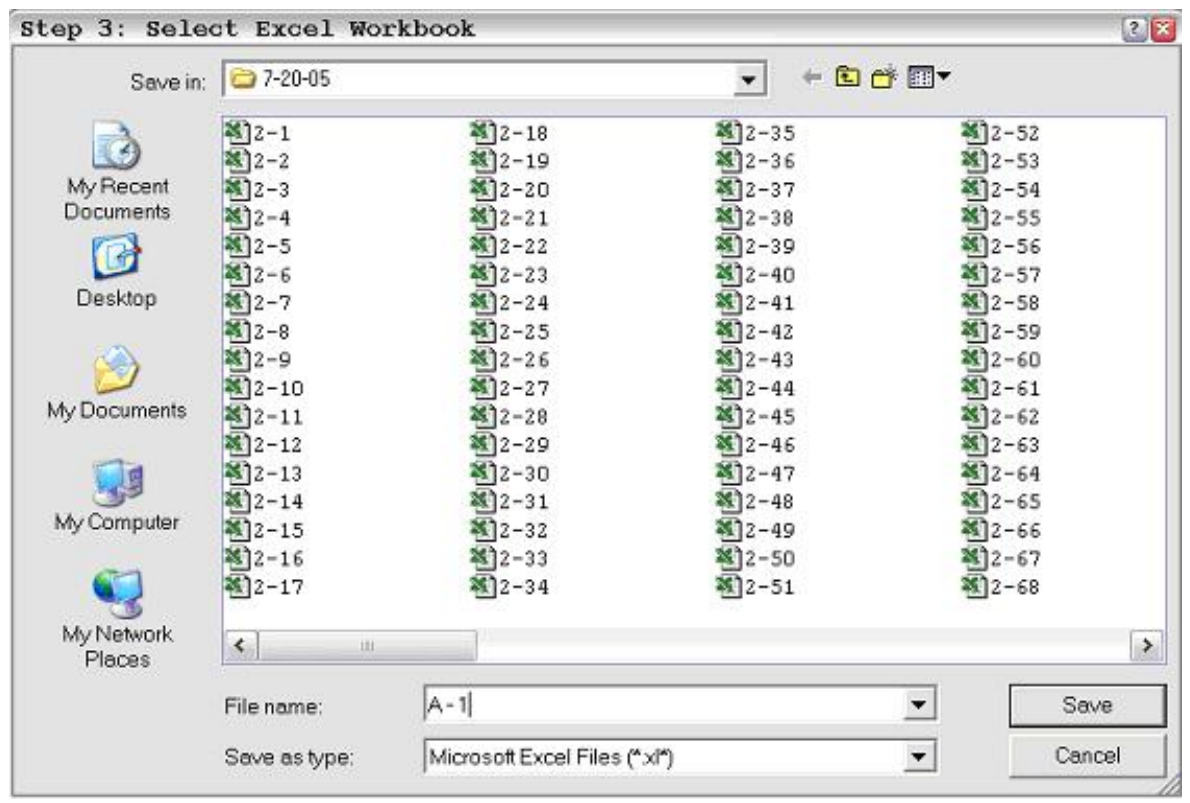

Figure A. 13 - Excel Exchange: Step 3 
Click on "Save" after step 3. A message will appear telling the end user that the operation was successful. After the files have been processed through excel exchange, the data will appear in an excel workbook as a number set of wavelengths and intensities. They can be plotted in excel using the plot wizard.

Dynamic LIBS testing involved a different process from binning. It is called cross sectioning. Cross sectioning is a function in Winspec32 that allows the user to look at a portion of data as a 2 dimensional graph with respect to intensity, as opposed to the normal view of the image. Cross sectioning allowed observation of the temporal behavior of the emission lines in dynamic LIBS testing.

Go to Process/Cross Section

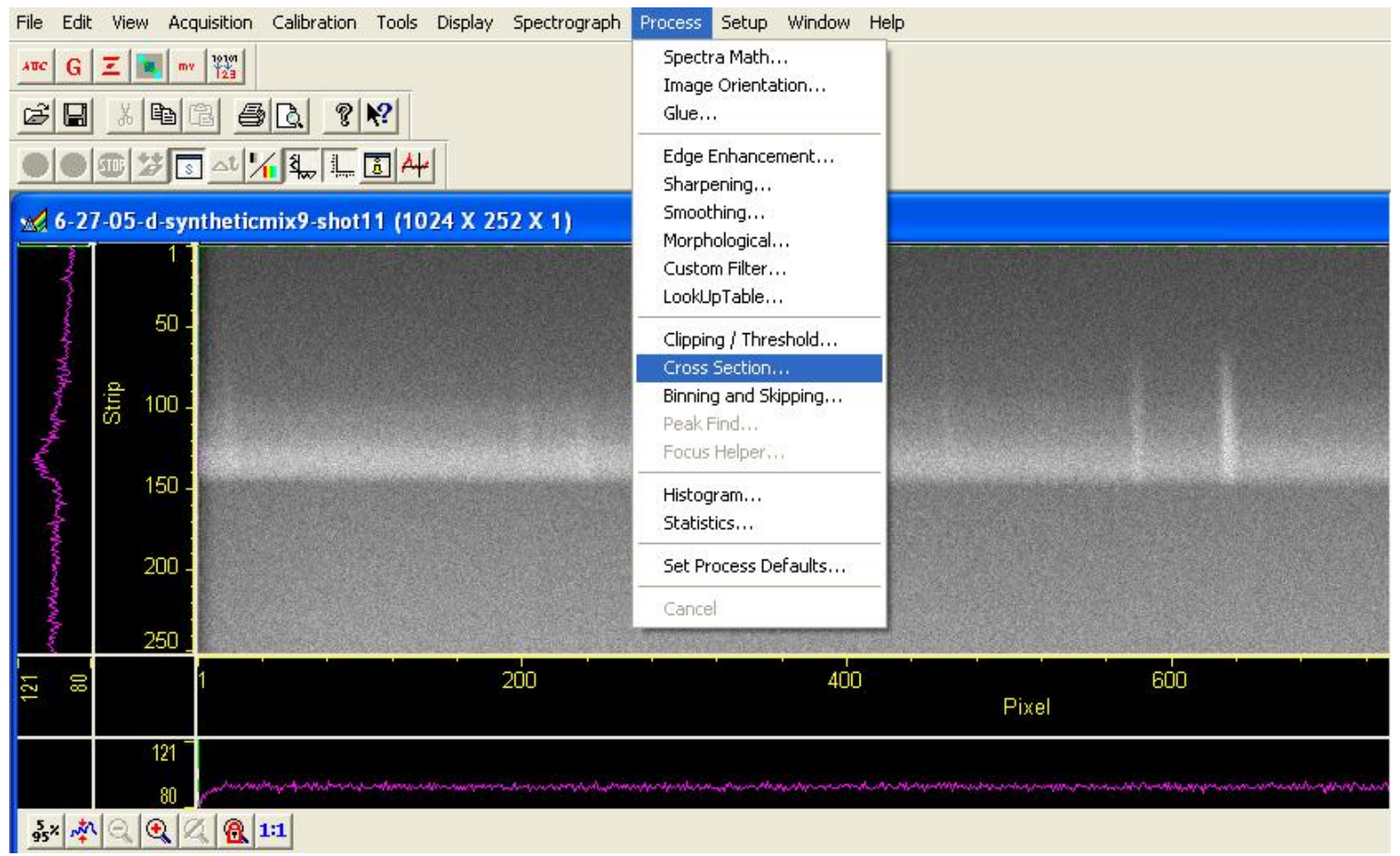

Figure A. 14 - Cross Section 
Select the filename to be cross sectioned

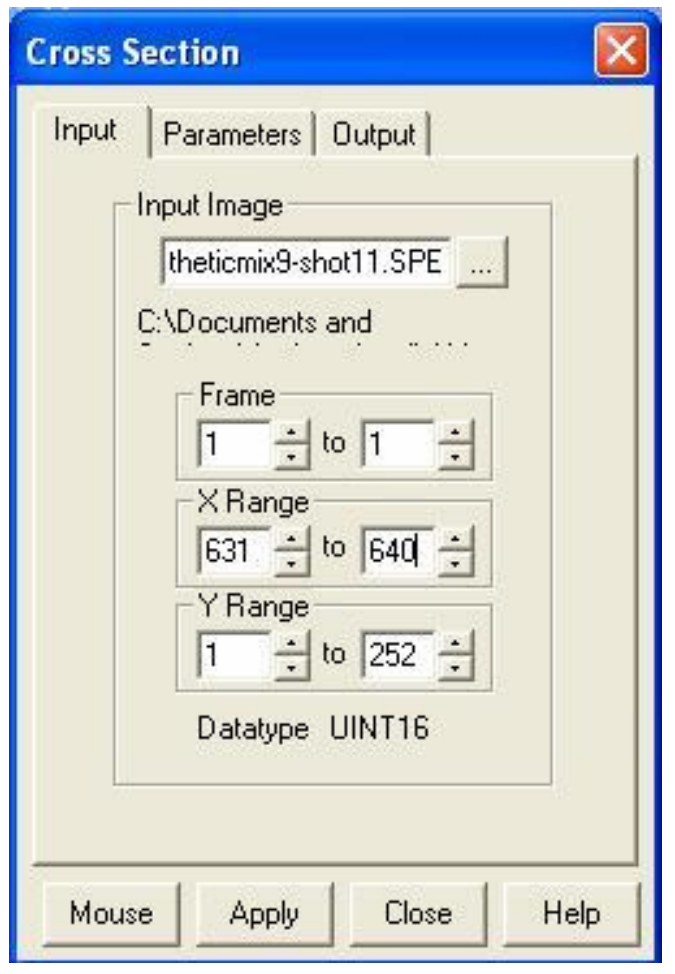

Figure A. 15 Input File

During selection of input, the software asks for a selection of the x-range and $y$ range. For analysis of the dynamic LIBS data, the Y range remained from 1 to 252 . The $\mathrm{X}$ range indicated the desired wavelength selected. Pixels 631 to 640 shown in Figure A.15 corresponded with the carbon-related 447nm line for this particular shot.

Select the parameters.

The selection of parameters differs from the binning and skipping process. To observe an individual emission line, a series of pixels were selected for the $\mathrm{X}$ range. To accurately represent the dynamic LIBS emission line, a mathematical average of these pixels was applied to a Y cross section as shown in Figure A.16. 


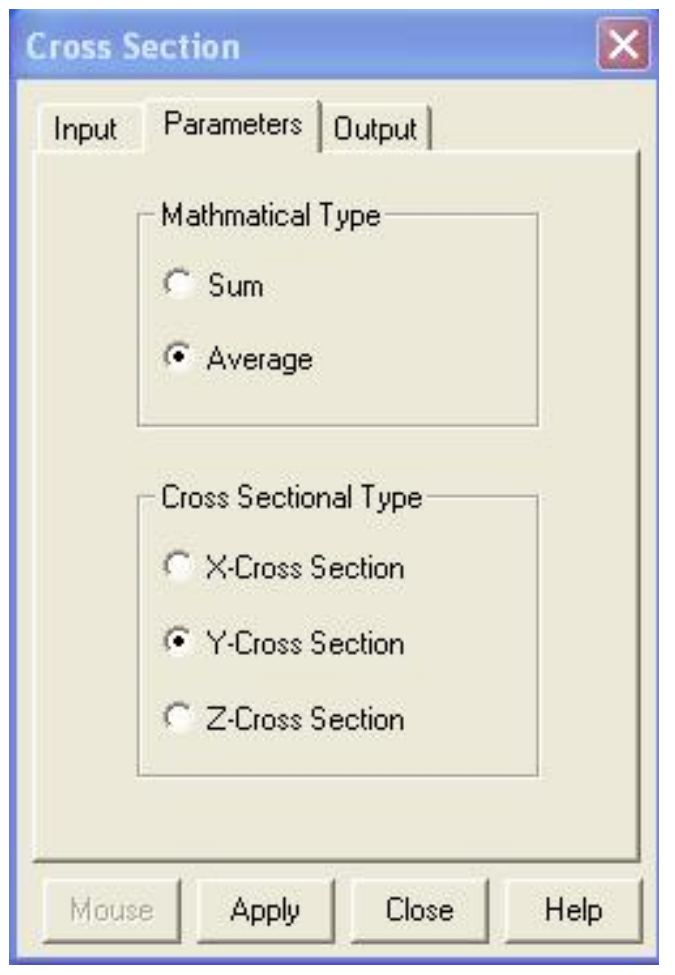

Figure A. 16 - Parameters of Cross Sectioning

Select the output filename

The output was selected to be saved as the mix number, the shot number and the emission line. For example, the carbon related emission line at $447 \mathrm{~nm}$ from mix 9, shot 11 was named “ mix9shot11C447”. 


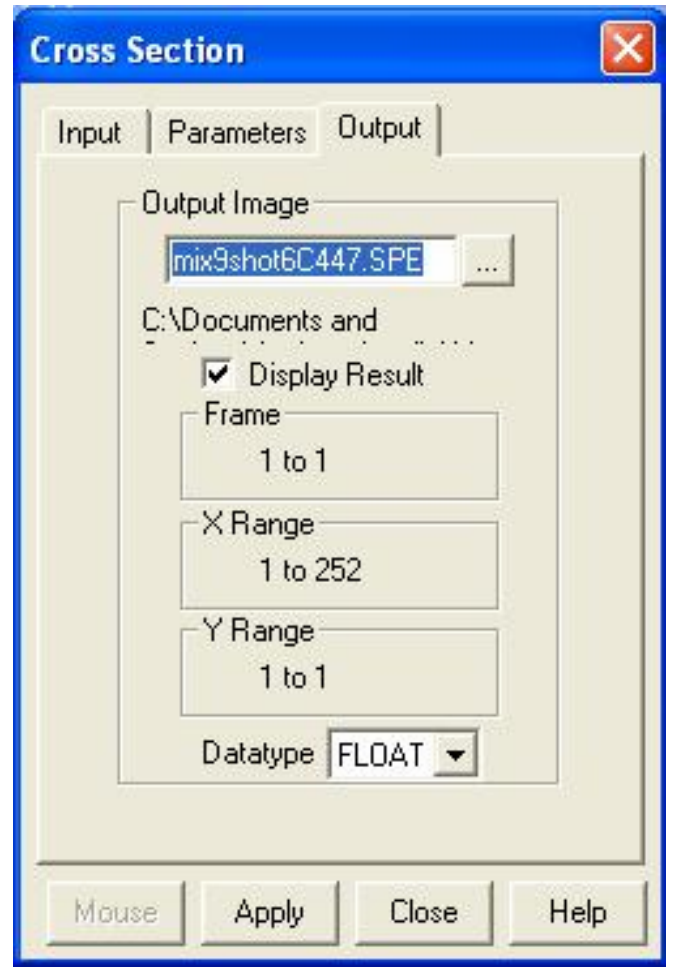

Figure A. 17 - Output File

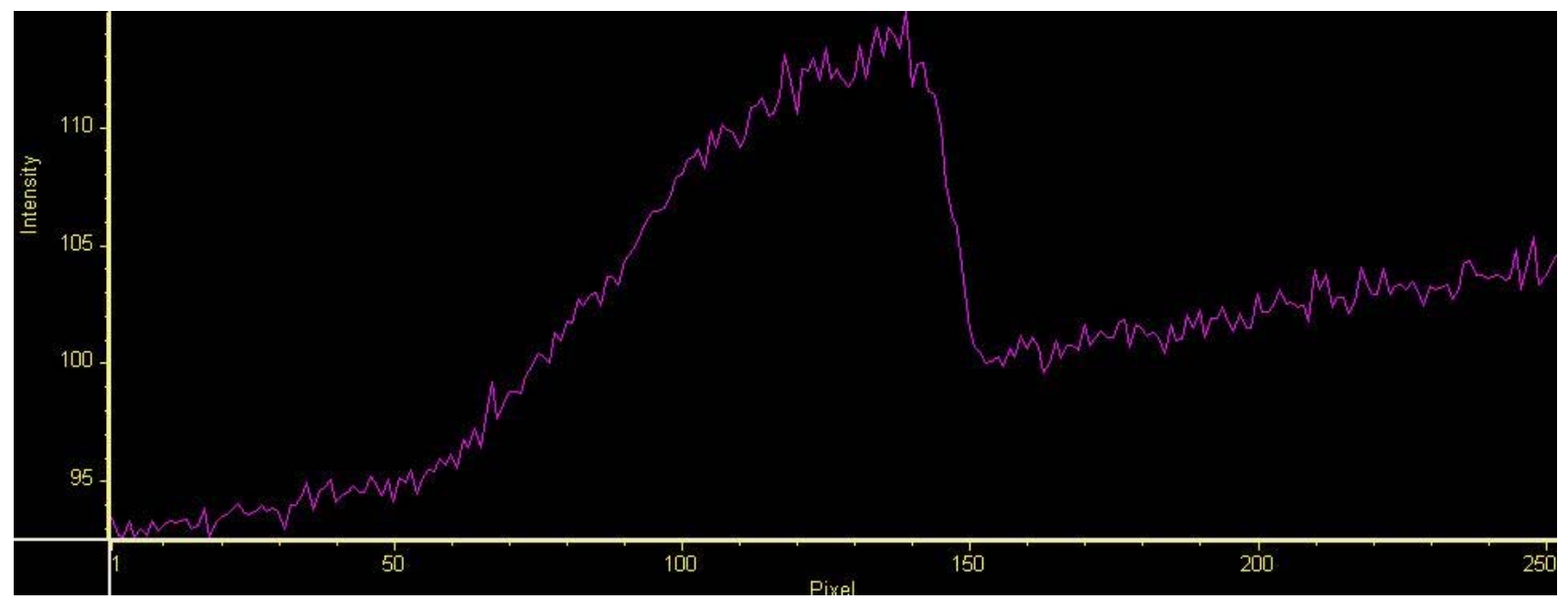

Figure A. 18 - mix9shot11C447 final product

After cross-sectioning, the data was then taken through excel exchange and plotted in Microsoft Excel. 


\section{APPENDIX B: FIGURES OF RAW DATA FROM STATIC LIBS TESTING}

The following collection of data represents the first 10 of 100 shots of each synthetic mixture. Displaying each individual shot would be excessive and not necessary. This appendix shows what the raw data from static LIBS testing looks like before processing. Each figure displays the first 10 shots of the synthetic mixtures. When reading the spectrum, lines are noticed at different wavelengths. These lines correspond to the different elements that are in the sample. Iron has lines at 432, 439, and $441 \mathrm{~nm}$. Carbon has two lines at 445 and 447 nanometers. Mercury contains a single emission line at $436 \mathrm{~nm}$. 
Synthetic Mix 1: 0.0770 g graphite, 0.0704g NIST 1635 Coal Fines, 0.0138g iron powder, $0.0108 g$ mercuric oxide
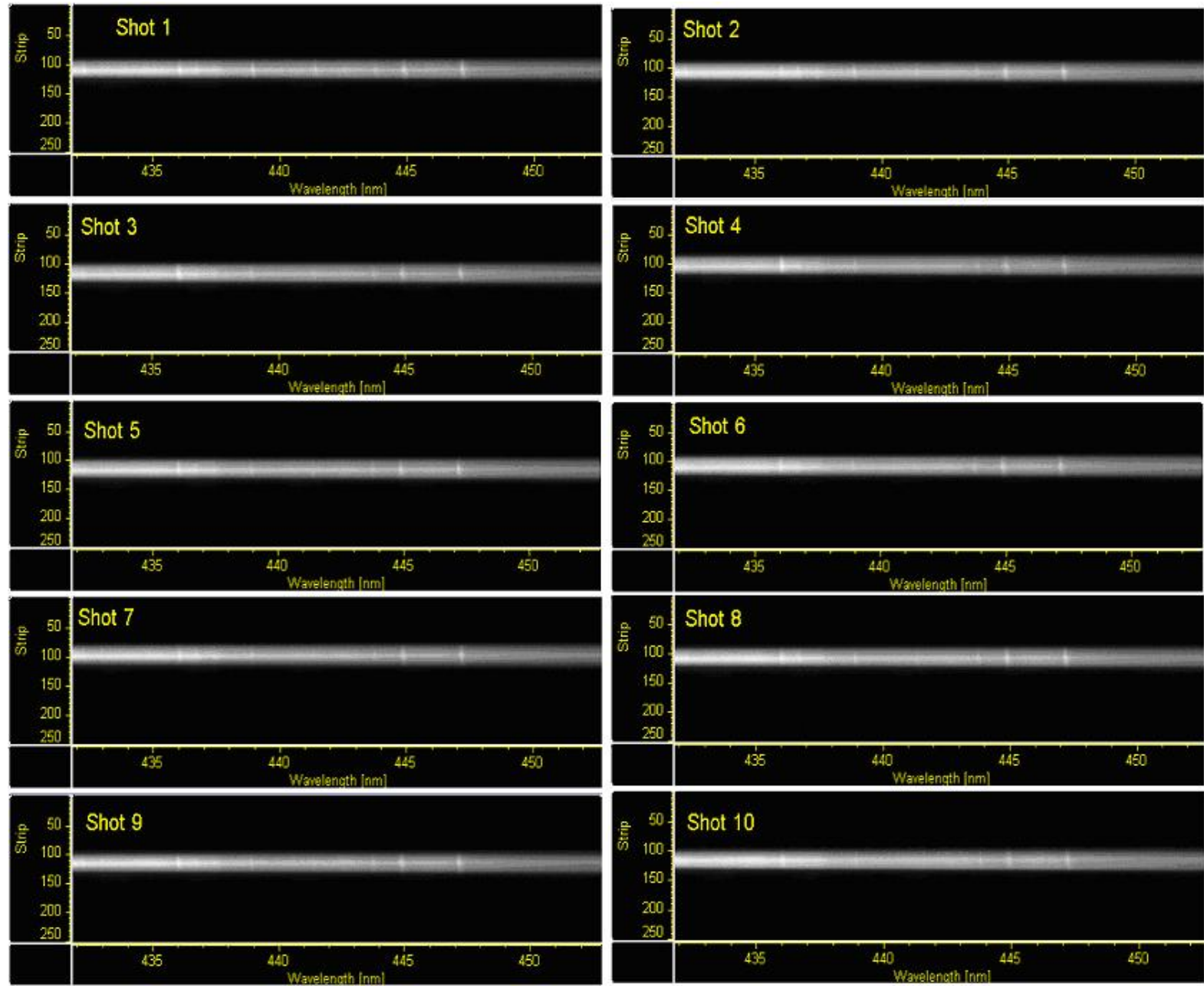

Figure B. 1 - First 10 shots of Synthetic Mix 1.

73.42\% Carbon, 8.12\% Iron, 5.81\% Mercury

\begin{tabular}{|l|l|r|r|}
\hline LINE & STDEV & RANGE & TOLERANCE \\
\hline FE432 & 636.8736 & 3825.5 & $16.65 \%$ \\
\hline HG436 & 1367.995 & 12953.5 & $10.56 \%$ \\
\hline FE439 & 1031.999 & 7186 & $14.36 \%$ \\
\hline FE441 & 525.5815 & 4159.5 & $12.64 \%$ \\
\hline C445 & 1215.274 & 9918 & $12.25 \%$ \\
\hline C447 & 1187.696 & 10553.5 & $11.25 \%$ \\
\hline
\end{tabular}

Table B. 1 - Mix 1 Tolerance 
Synthetic Mix 2: 0.3344g graphite, 0 g NIST 1635 coal fines, 0.006 g iron powder, 0.0026 g mercuric oxide
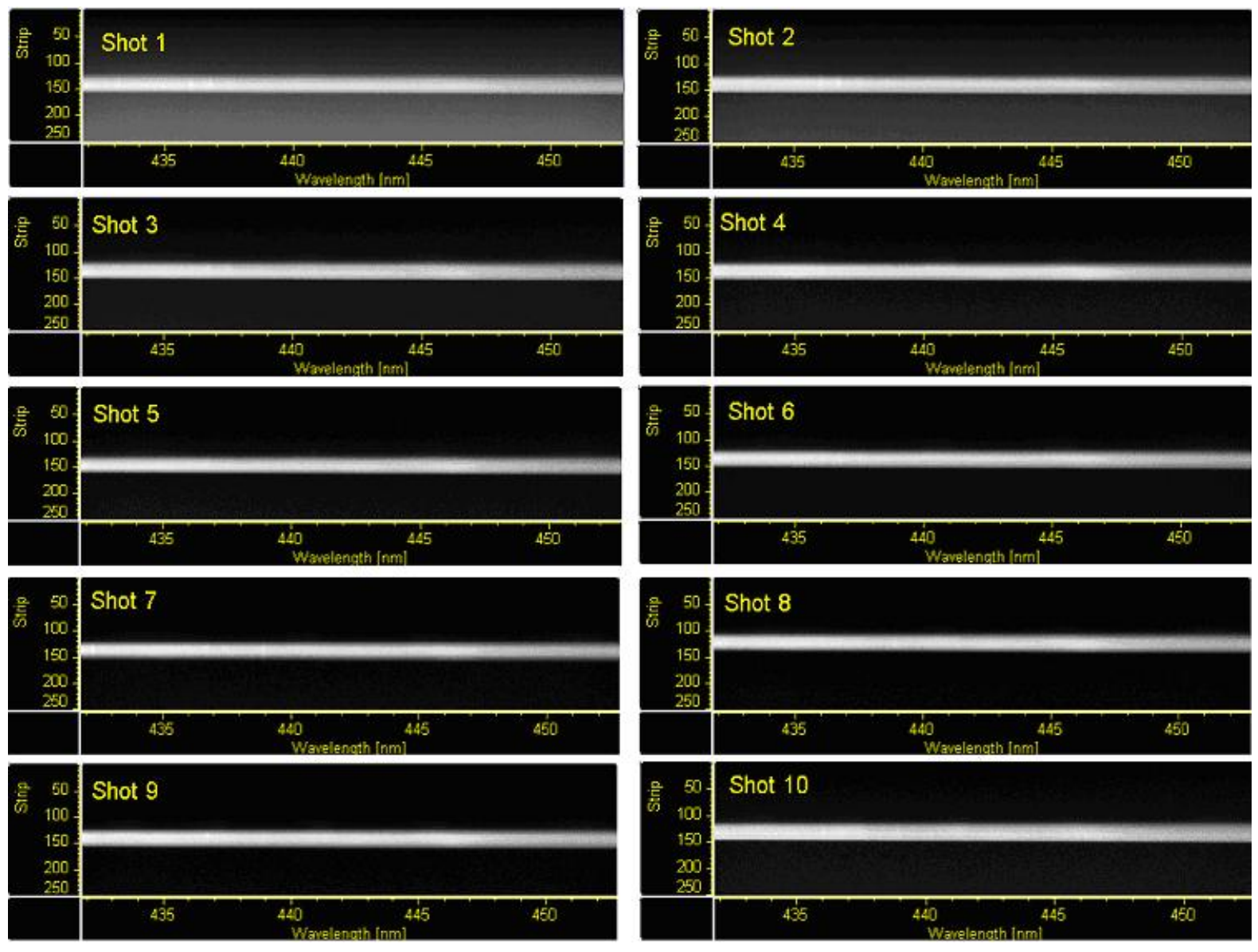

Figure B. 2 - First 10 shots of Synthetic Mix 2.

97.49\% Carbon, 1.75\% Iron, 0.7\% Mercury

\begin{tabular}{|l|l|r|r|}
\hline LINE & STDEV & RANGE & TOLERANCE \\
\hline FE432 & 337.2831 & 1404.5 & $24.01 \%$ \\
\hline HG436 & 1433.986 & 8511.5 & $16.85 \%$ \\
\hline FE441 & 308.8991 & 1855 & $16.65 \%$ \\
\hline FE439 & 283.1096 & 847 & $33.42 \%$ \\
\hline
\end{tabular}

Table B. 2 - Mix 2 Tolerance 
Synthetic Mix 3: 0 g graphite, 0.7575 g NIST 1635 coal fines, 0.0998 g iron powder, 0.0111 g mercuric oxide
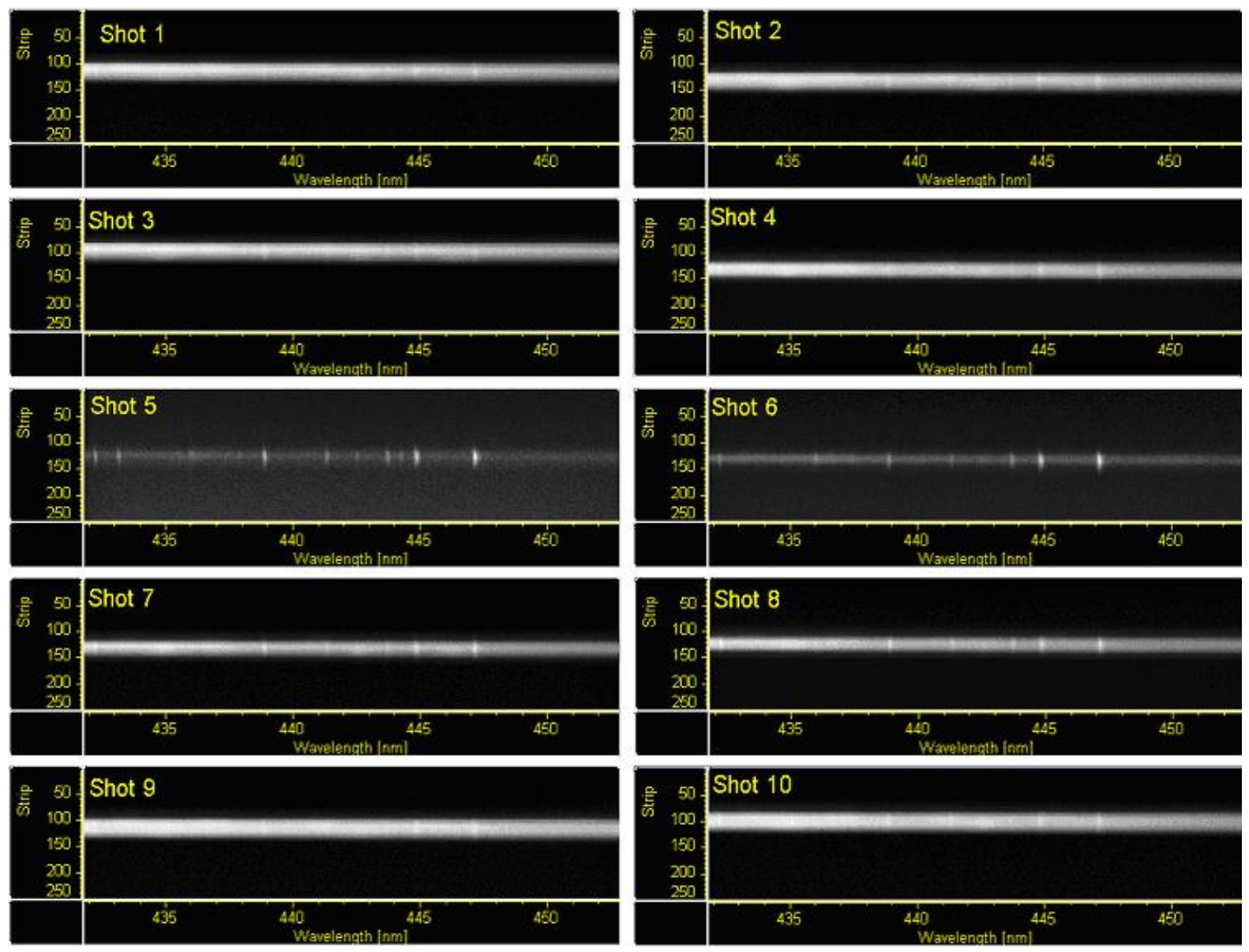

Figure B. 3 - First 10 shots of Synthetic Mix 3

61.06\% Carbon, 11.7\% Iron, 1.18\% Mercury

\begin{tabular}{|l|r|r|r|}
\hline LINE & \multicolumn{1}{|c|}{ STDEV } & RANGE & TOLERANCE \\
\hline FE432 & 482.8996 & 2041.5 & $23.65 \%$ \\
\hline HG436 & 211.1772 & 1248.5 & $16.91 \%$ \\
\hline FE439 & 720.2086 & 3140 & $22.94 \%$ \\
\hline FE441 & 371.8231 & 1629 & $22.83 \%$ \\
\hline C445 & 485.4924 & 2755.5 & $17.62 \%$ \\
\hline C447 & 533.744 & 2654.5 & $20.11 \%$ \\
\hline
\end{tabular}

Table B. 3 - Mix 3 Tolerance 
Synthetic Mix 4: 0.0082 g graphite, 0.3113 g NIST 1635 coal fines, 0.0199 g iron

powder, $0.0019 \mathrm{~g}$ mercuric oxide
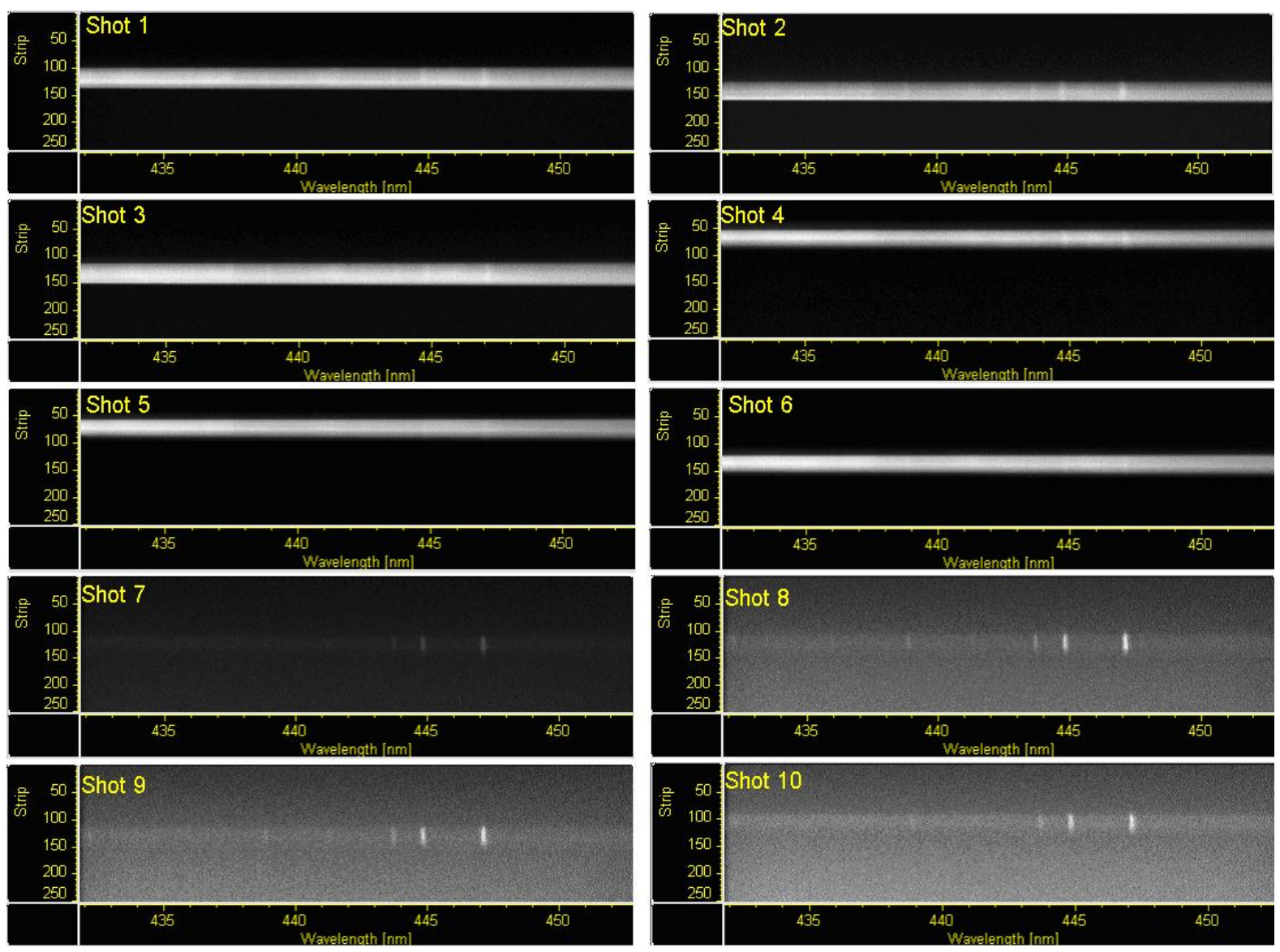

Figure B. 4 - First 10 shots of Synthetic Mix 4

66.24\% Carbon, 6.05\% Iron, 0.52\% Mercury

\begin{tabular}{|l|l|r|r|}
\hline LINE & STDEV & RANGE & TOLERANCE \\
\hline FE432 & 63.42216 & 537.5 & $11.80 \%$ \\
\hline FG436 & 58.25787 & 327.5 & $17.79 \%$ \\
\hline FE439 & 59.88799 & 312.5 & $19.16 \%$ \\
\hline FE441 & 80.74048 & 450 & $17.94 \%$ \\
\hline C445 & 241.1991 & 1593 & $15.14 \%$ \\
\hline C447 & 225.1583 & 1435 & $15.69 \%$ \\
\hline
\end{tabular}

Table B. 4 - Mix 4 Tolerance 
Synthetic Mix 5: 0.0936 g graphite, 0.2985 g NIST 1635 coal fines, 0.0213 g iron

powder, 0.0003 g mercuric oxide
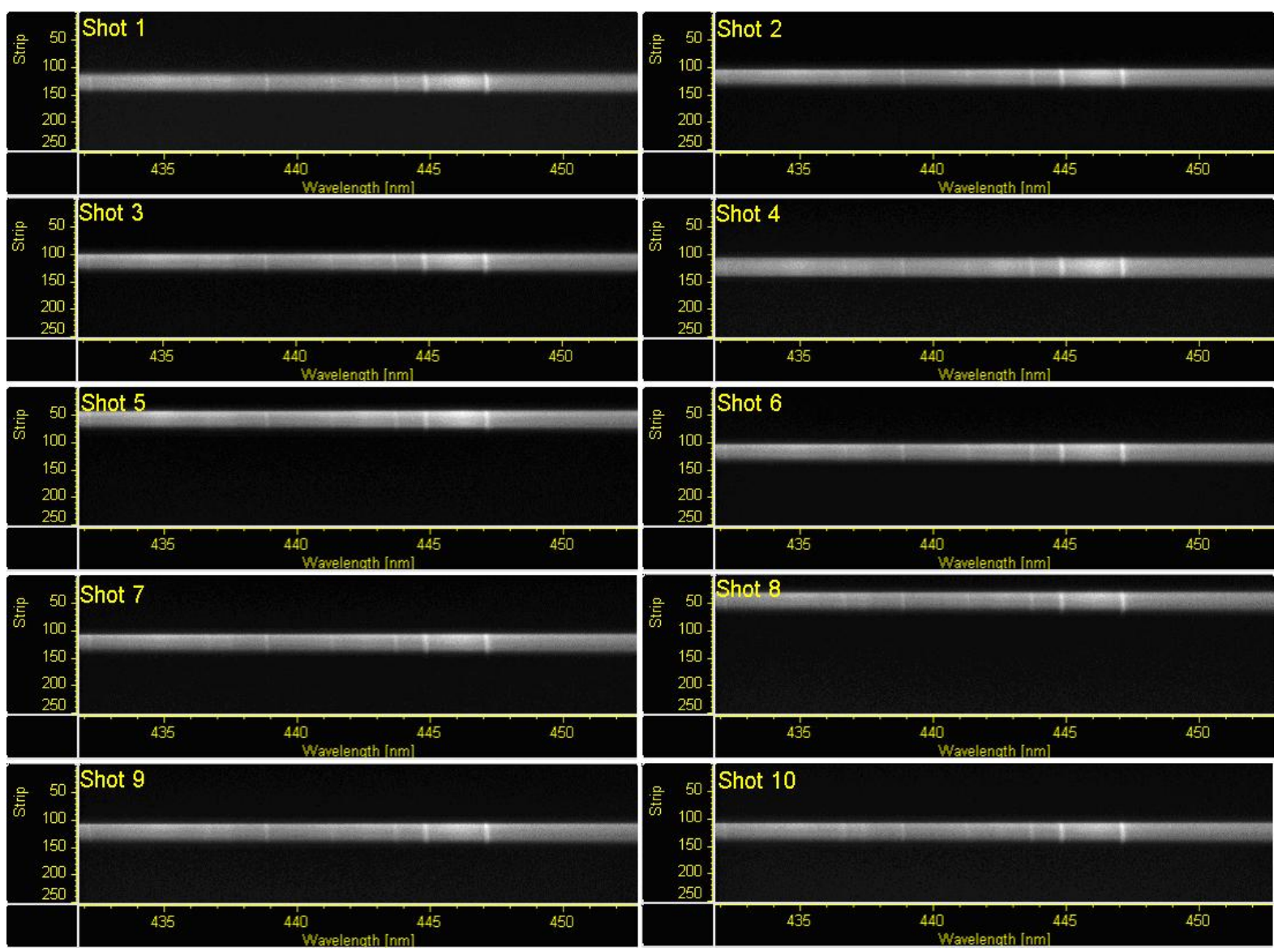

Figure B. 5 - First 10 shots of Synthetic Mix 5

73.12\% Carbon, 5.32\% Iron, 0.08\% Mercury

\begin{tabular}{|l|l|r|r|}
\hline LINE & STDEV & RANGE & TOLERANCE \\
\hline FE432 & 186.3826 & 1050 & $17.75 \%$ \\
\hline HG436 & 51.08251 & 310.5 & $16.45 \%$ \\
\hline FE439 & 240.2219 & 1013 & $23.71 \%$ \\
\hline FE441 & 132.0769 & 567 & $23.29 \%$ \\
\hline C445 & 451.6759 & 2177 & $20.75 \%$ \\
\hline C447 & 434.8764 & 2081 & $20.90 \%$ \\
\hline
\end{tabular}

Table B. 5 - Mix 5 Tolerance 
Synthetic Mix 6: 0 g graphite, 0.2967 g NIST 1635 coal fines, 0.0213 g iron powder, 0.0084 g mercuric oxide
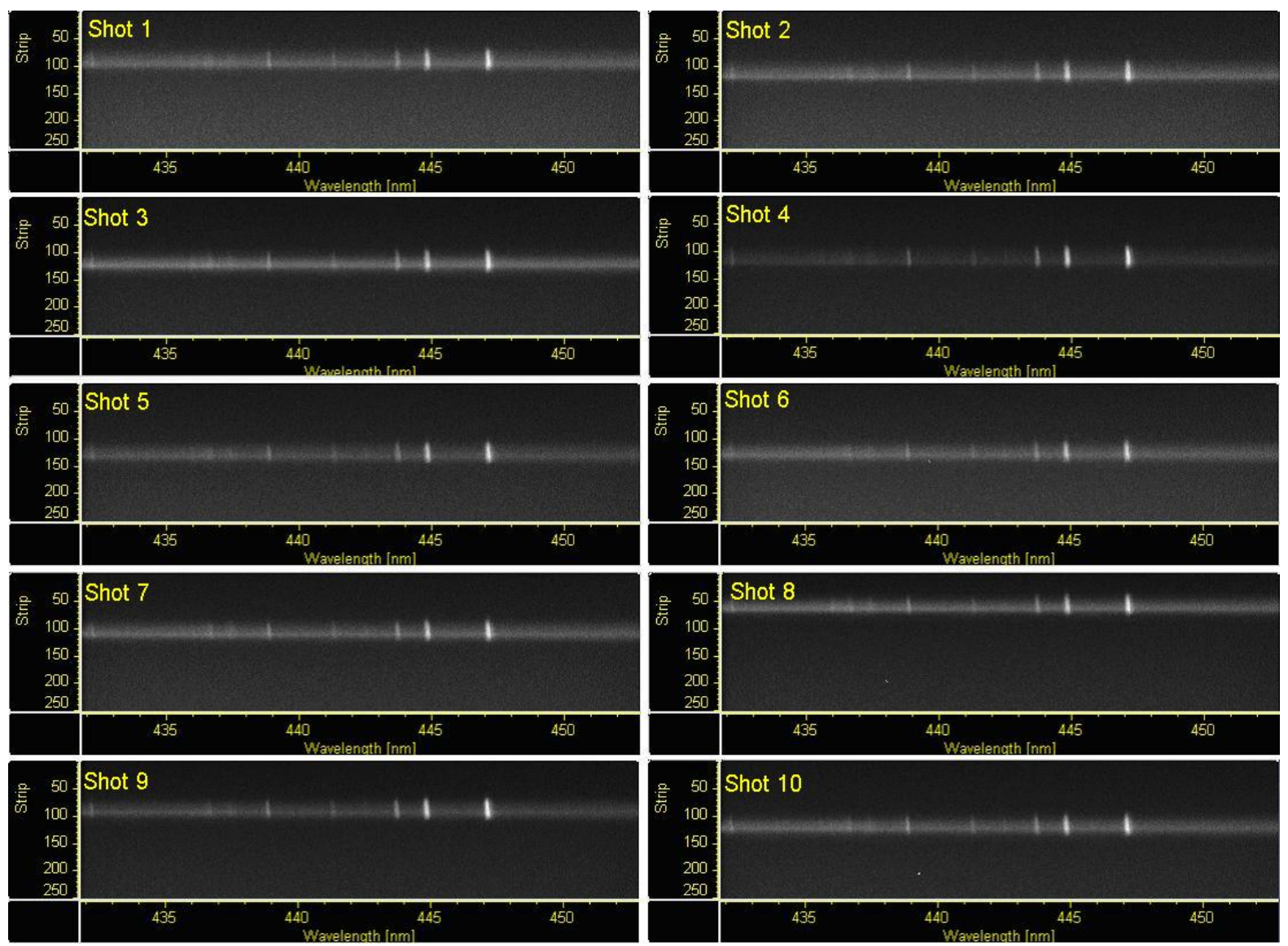

Figure B. 6 - First 10 shots of Synthetic Mix 6

67.75\% Carbon, 3.18\% Iron, 0.25\% Mercury

\begin{tabular}{|l|r|r|r|}
\hline LINE & \multicolumn{1}{|c|}{ STDEV } & RANGE & TOLERACE \\
\hline FE432 & 214.142 & 1128 & $18.98 \%$ \\
\hline HG436 & 45.30804 & 245 & $18.49 \%$ \\
\hline FE439 & 326.205 & 1384 & $23.57 \%$ \\
\hline FE441 & 171.6206 & 826.5 & $20.76 \%$ \\
\hline C445 & 641.4904 & 2394 & $26.80 \%$ \\
\hline C447 & 656.6691 & 2422 & $27.11 \%$ \\
\hline
\end{tabular}

Table B. 6 - Mix 6 Tolerance 
Synthetic Mix 7: 0.1519 g graphite, 0.2415 g NIST 1635 coal fines, 0 g iron powder, 0.0132 g mercuric oxide
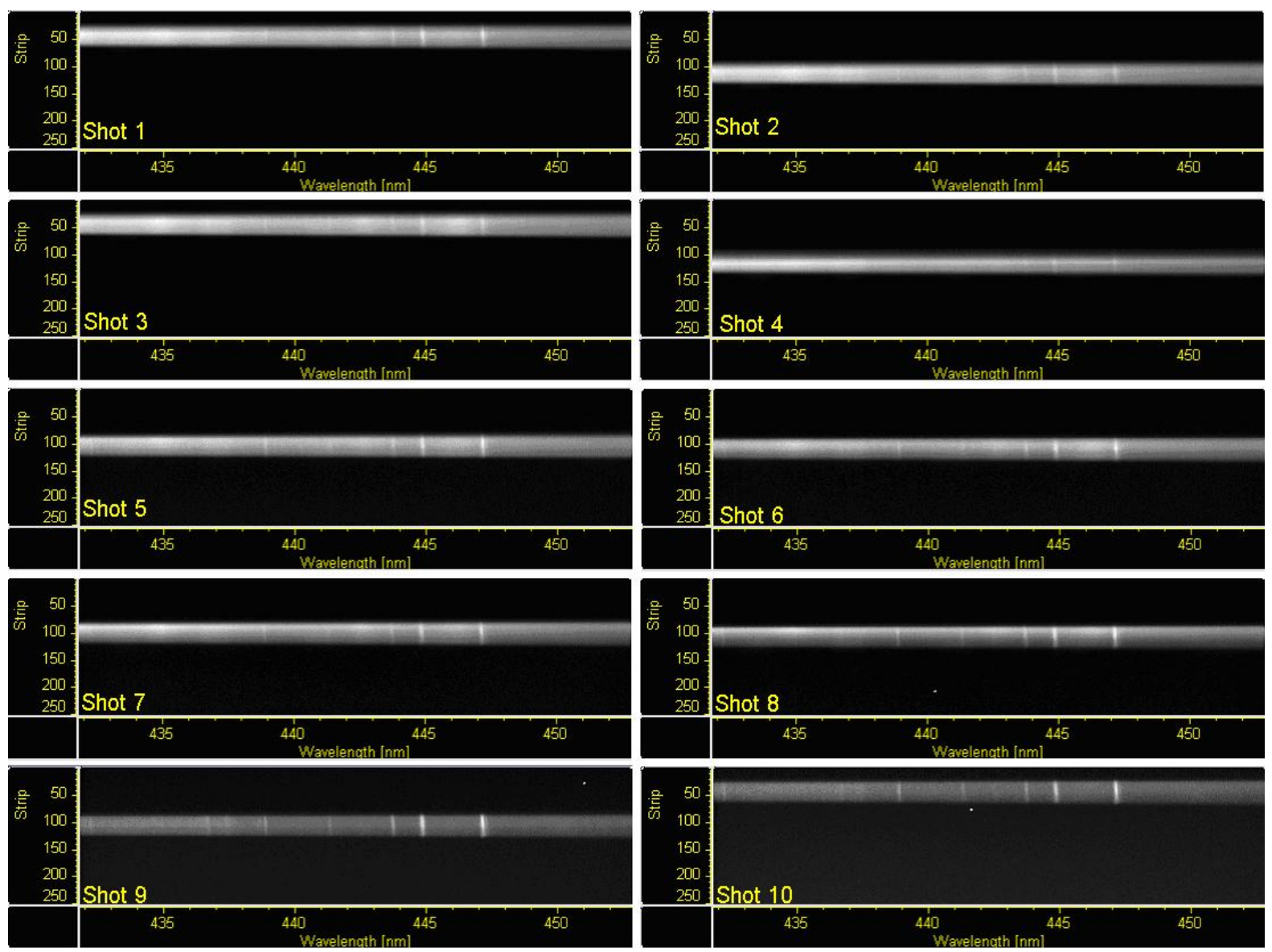

Figure B. 7 - First 10 shots of Synthetic Mix 7

81.2\% Carbon, 0.15\% Iron, 0.31\% Mercury

\begin{tabular}{|l|l|r|r|}
\hline LINE & STDEV & RANGE & TOLERANCE \\
\hline FE432 & 147.9022 & 1084 & $13.64 \%$ \\
\hline HG436 & 92.64634 & 785.5 & $11.79 \%$ \\
\hline FE439 & 349.9984 & 1846.5 & $18.95 \%$ \\
\hline FE441 & 174.5414 & 1112.5 & $15.69 \%$ \\
\hline C445 & 1032.066 & 6426 & $16.06 \%$ \\
\hline C447 & 1081.515 & 6669.5 & $16.22 \%$ \\
\hline
\end{tabular}

Table B. 7 - Mix 7 Tolerance 
Synthetic Mix 8: 0.2259 g graphite, 0.2121 g NIST 1635 coal fines, 0.0017 g iron

powder, $0.1497 \mathrm{~g}$ mercuric oxide
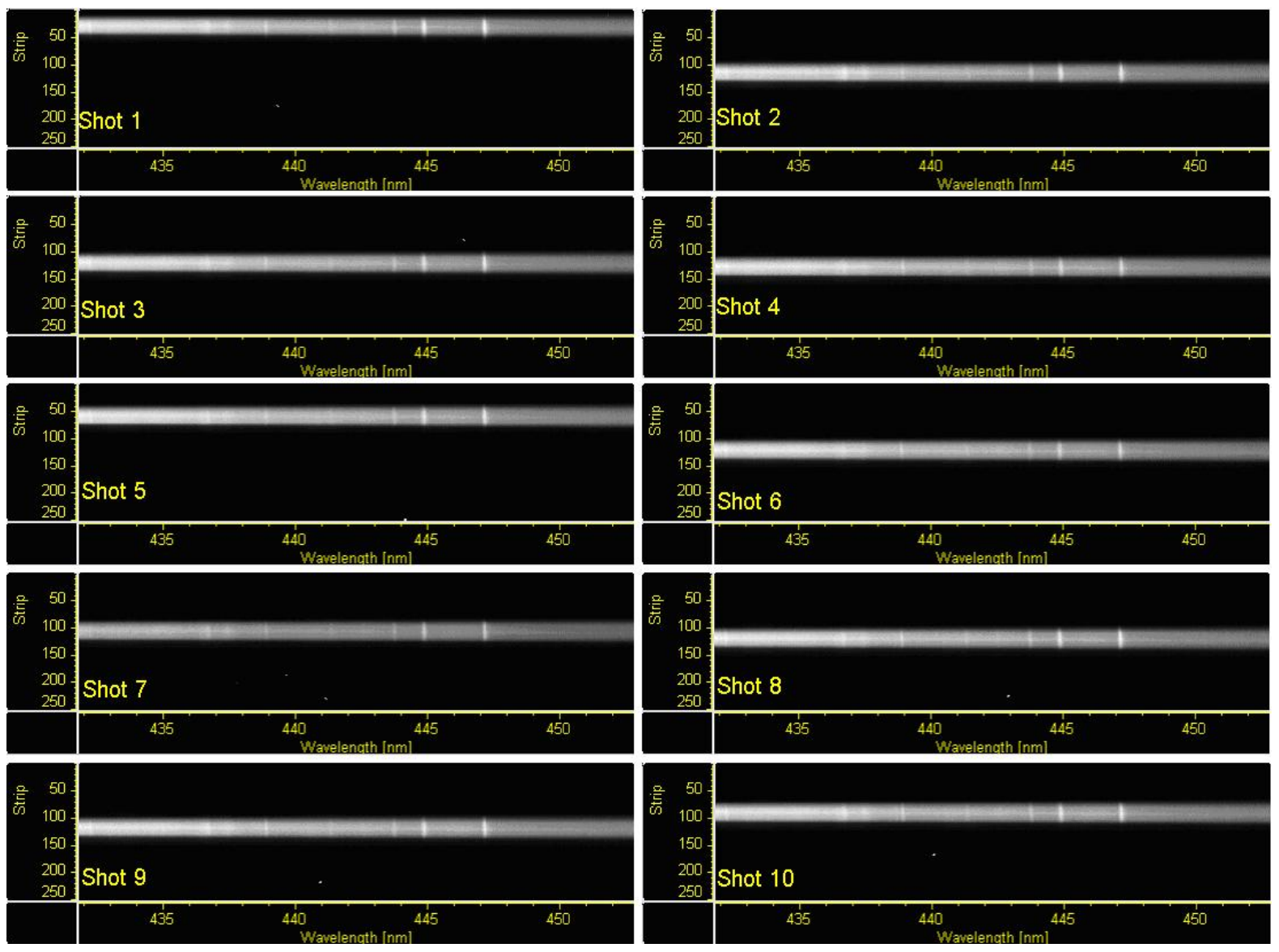

Figure B. 8 - First 10 shots of Synthetic Mix 8

81.21\% Carbon, 0.46\% Iron, 2.77\% Mercury

\begin{tabular}{|l|l|r|r|}
\hline LINE & STDEV & RANGE & TOLERANCE \\
\hline FE432 & 529.5033 & 2261.5 & $23.41 \%$ \\
\hline HG436 & 539.1545 & 2942.5 & $18.32 \%$ \\
\hline FE439 & 968.1487 & 4795 & $20.19 \%$ \\
\hline FE441 & 434.8169 & 2283.5 & $19.04 \%$ \\
\hline C445 & 2225.656 & 13204 & $16.86 \%$ \\
\hline C447 & 2606.168 & 15253.5 & $17.09 \%$ \\
\hline
\end{tabular}

Table B. 8 - Mix 8 Tolerance 
Synthetic Mix 9: 0.1757 g graphite, 0.2715 g NIST 1635 coal fines, 0.0072 g iron powder, $0.0138 \mathrm{~g}$ mercuric oxide
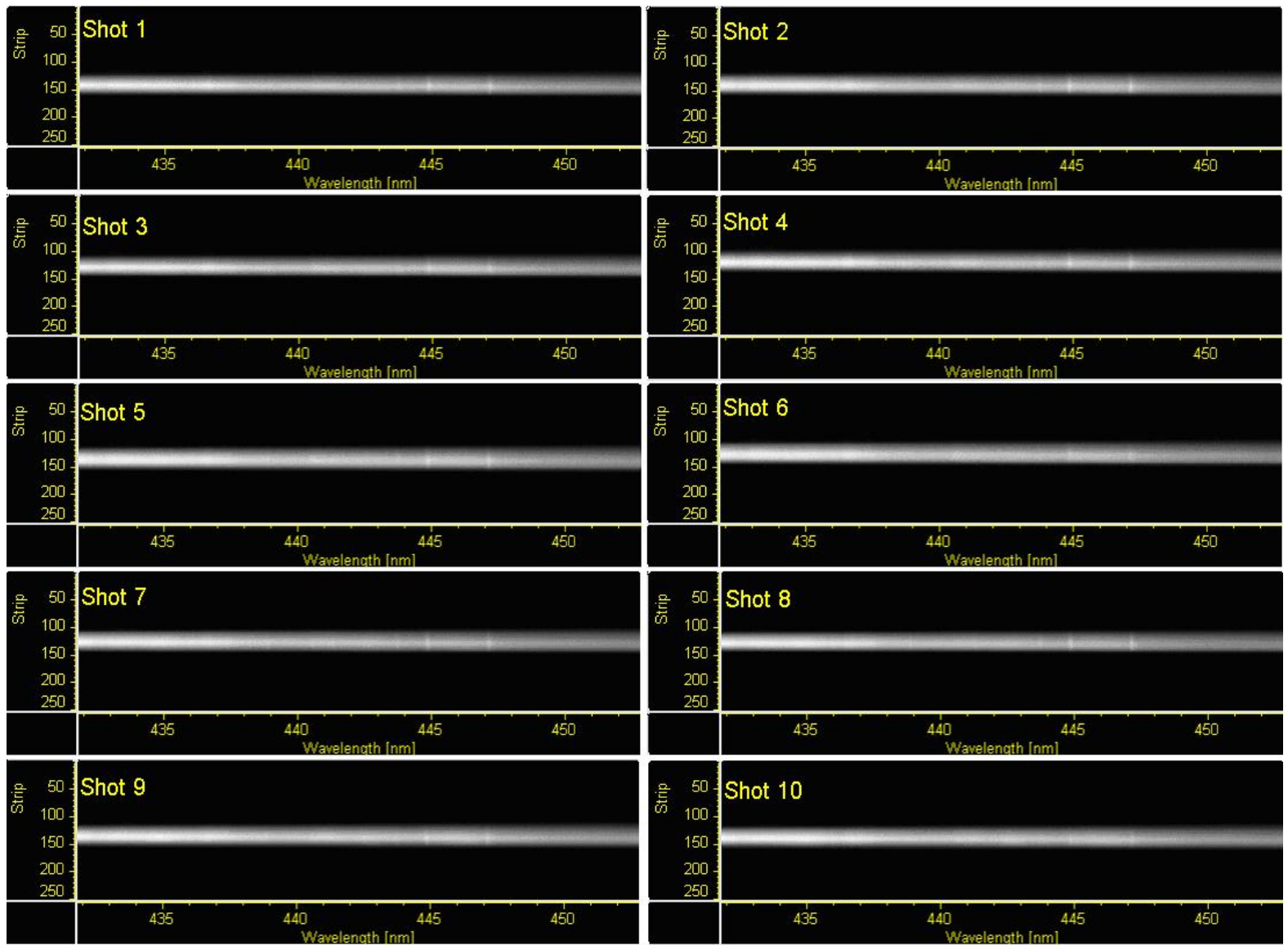

Figure B. 9 - First 10 shots of Synthetic Mix 9

78.11\% Carbon, 1.68\% Iron, 2.74\% Mercury

\begin{tabular}{|l|r|r|r|}
\hline LINE & STDEV & RANGE & TOLERANCE \\
\hline FE432 & 198.8775 & 1627 & $12.22 \%$ \\
\hline HG436 & 318.1999 & 1400.5 & $22.72 \%$ \\
\hline FE439 & 296.1578 & 2361 & $12.54 \%$ \\
\hline FE441 & 231.9034 & 1490 & $15.56 \%$ \\
\hline C445 & 726.731 & 3755 & $19.35 \%$ \\
\hline C447 & 702.3272 & 3987.5 & $17.61 \%$ \\
\hline
\end{tabular}

Table B. 9 - Mix 9 Tolerance 


\section{APPENDIX C: PROCESSED DATA FILES FROM STATIC LIBS TESTING}

This appendix shows processed data files from static LIBS. Each data file shown is a 100 shot average of a synthetic mixture. The procedure for processing the raw data (shown in appendix B) to this data is shown in appendix A. Each file was binned and converted into excel. From excel, the files were averaged and plotted. Places of the emission lines can be shown in figure 4.3.

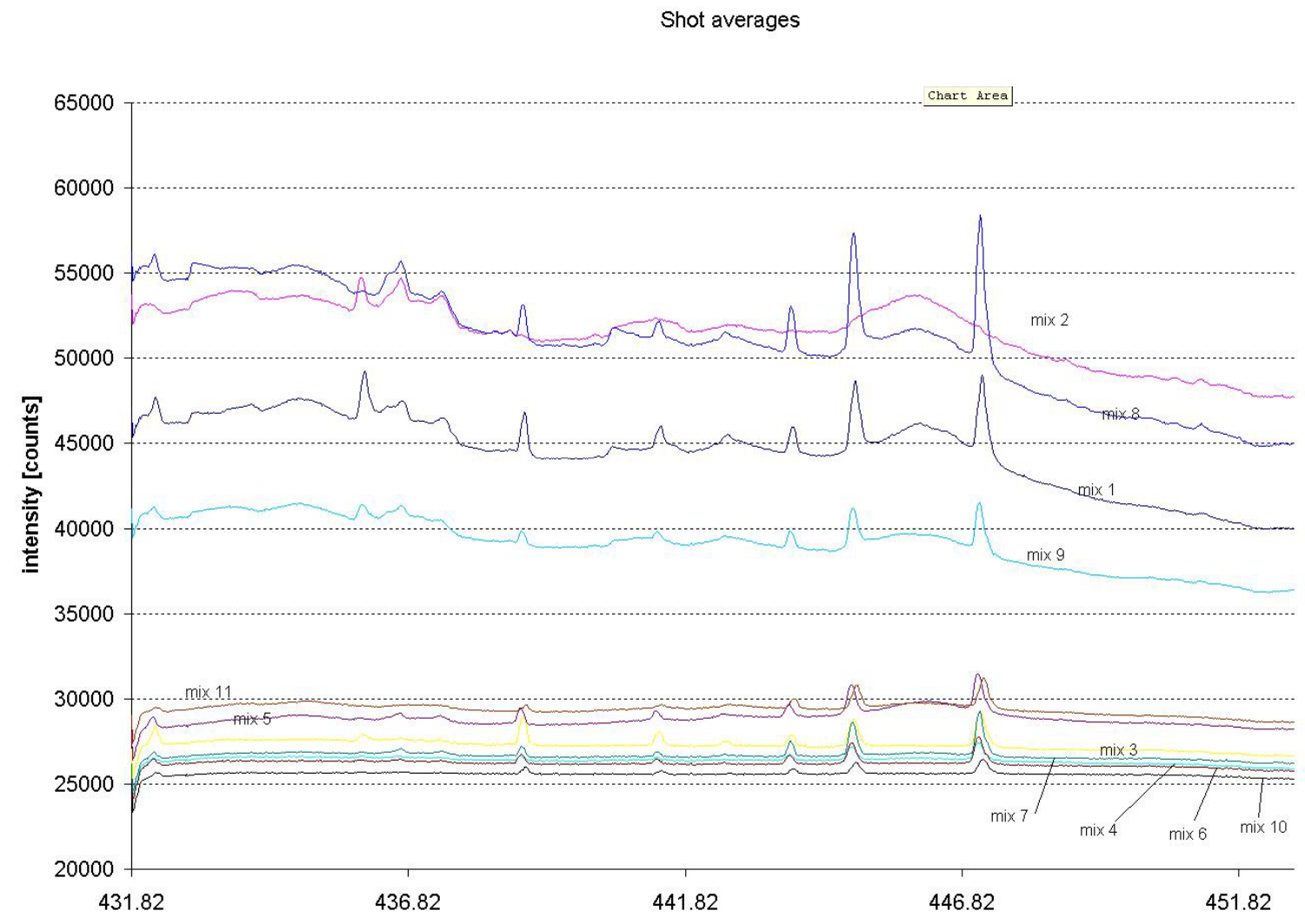

Figure C. 1 - 100 shot averages 
MIX 1

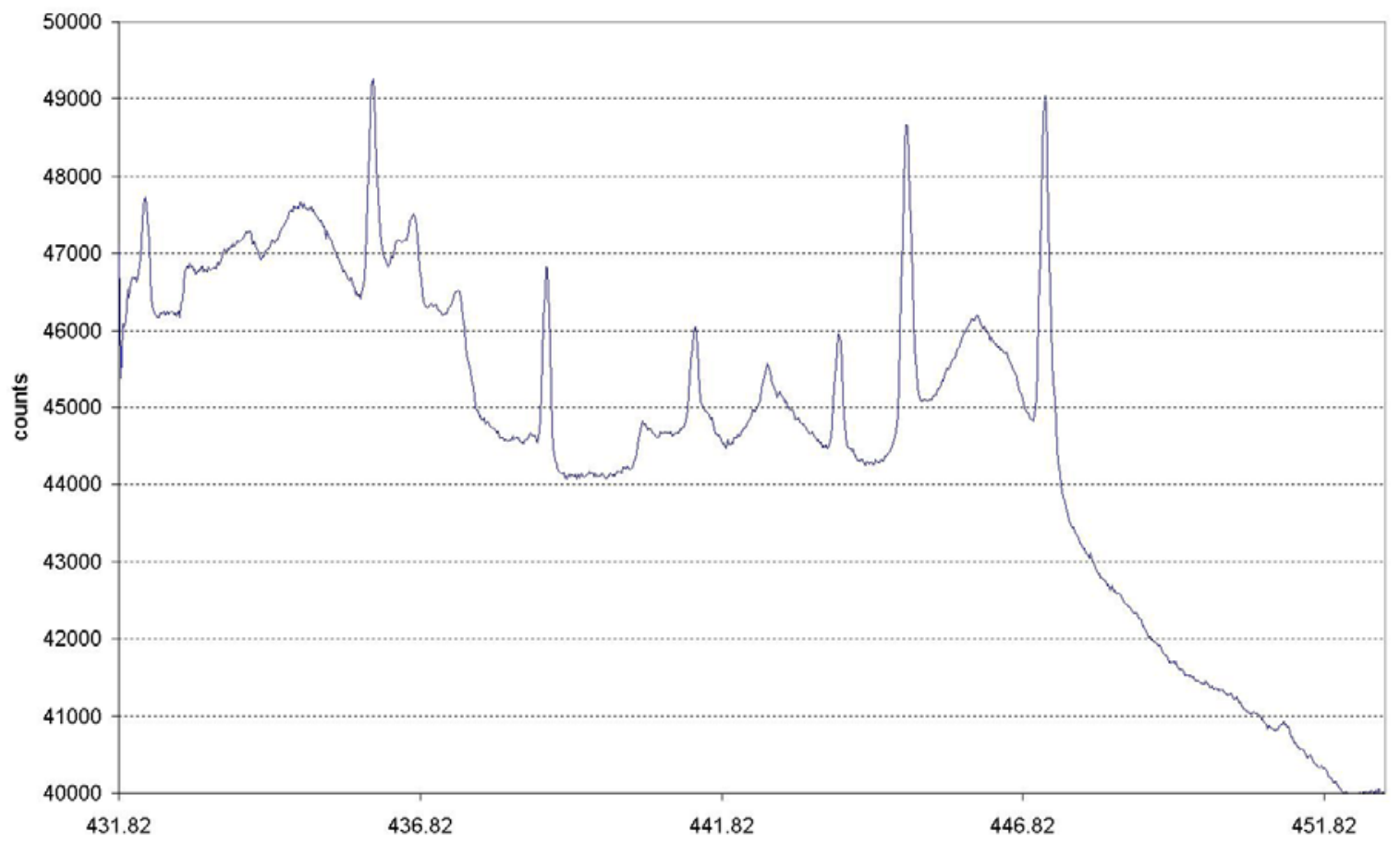

Figure C. 2 - 100 shot average of Mix 1

MIX 2

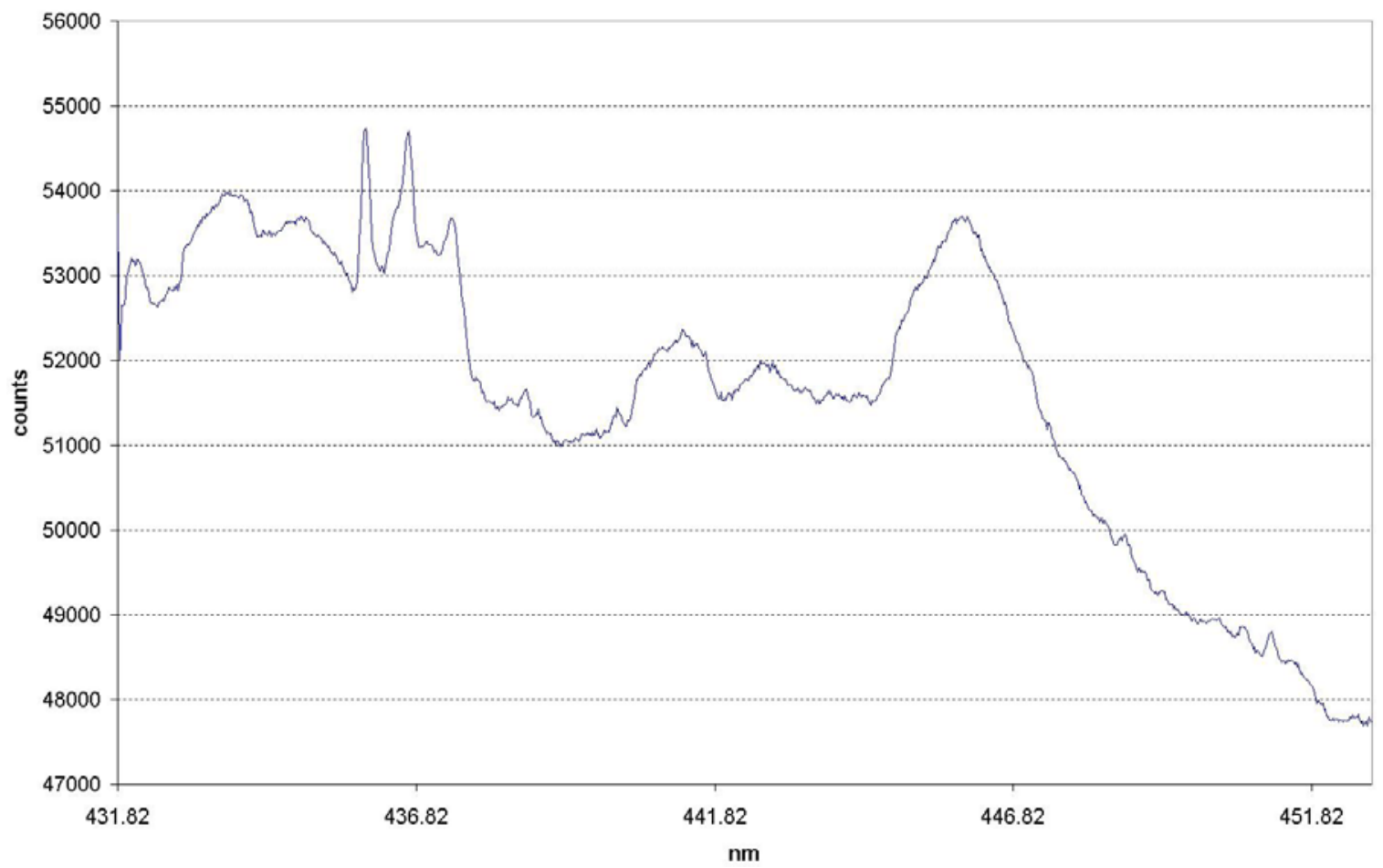

Figure C. 3 - 100 shot average of Mix 2 
MIX 3

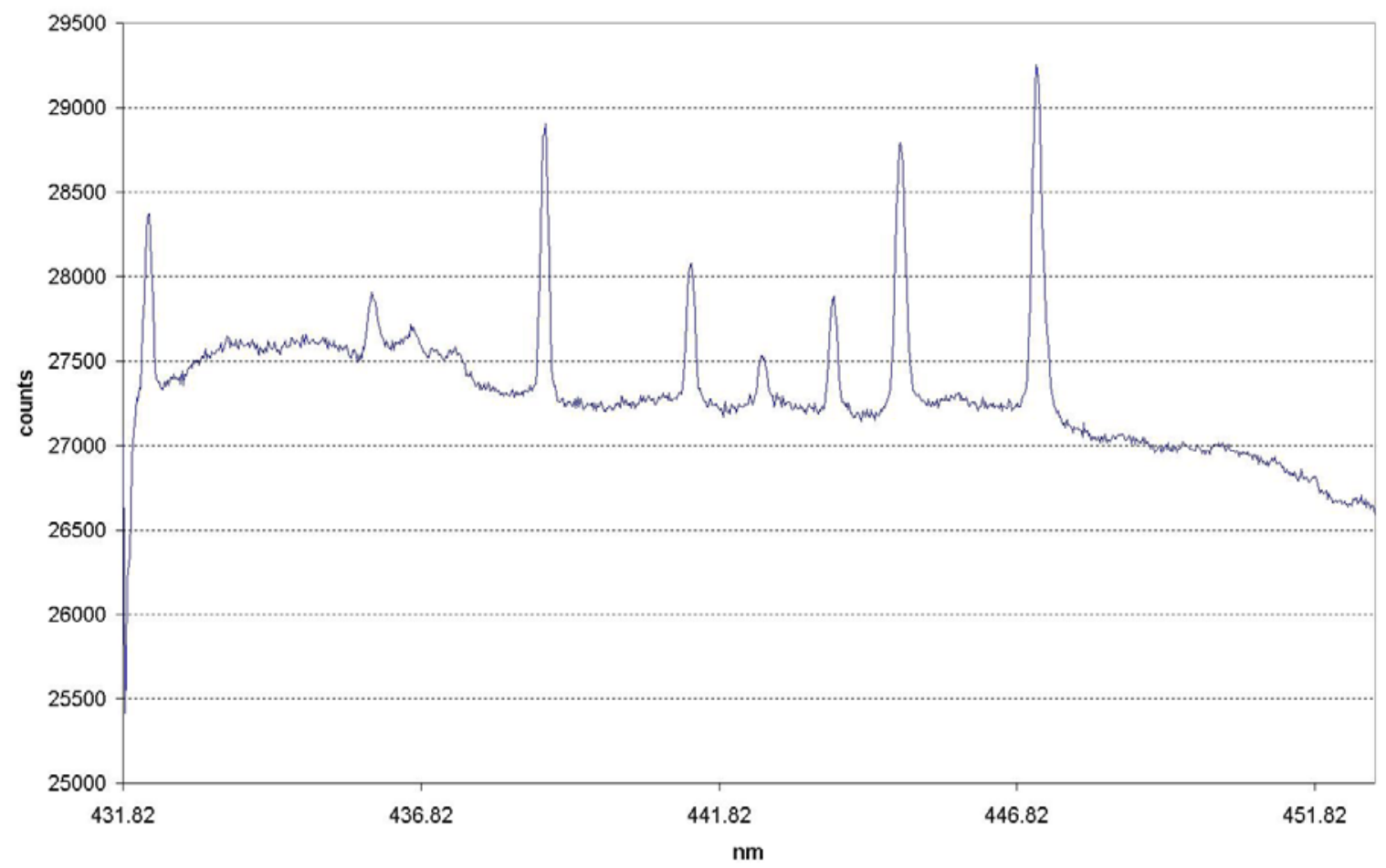

Figure C. 4 - 100 shot average of Mix 3

MIX 4

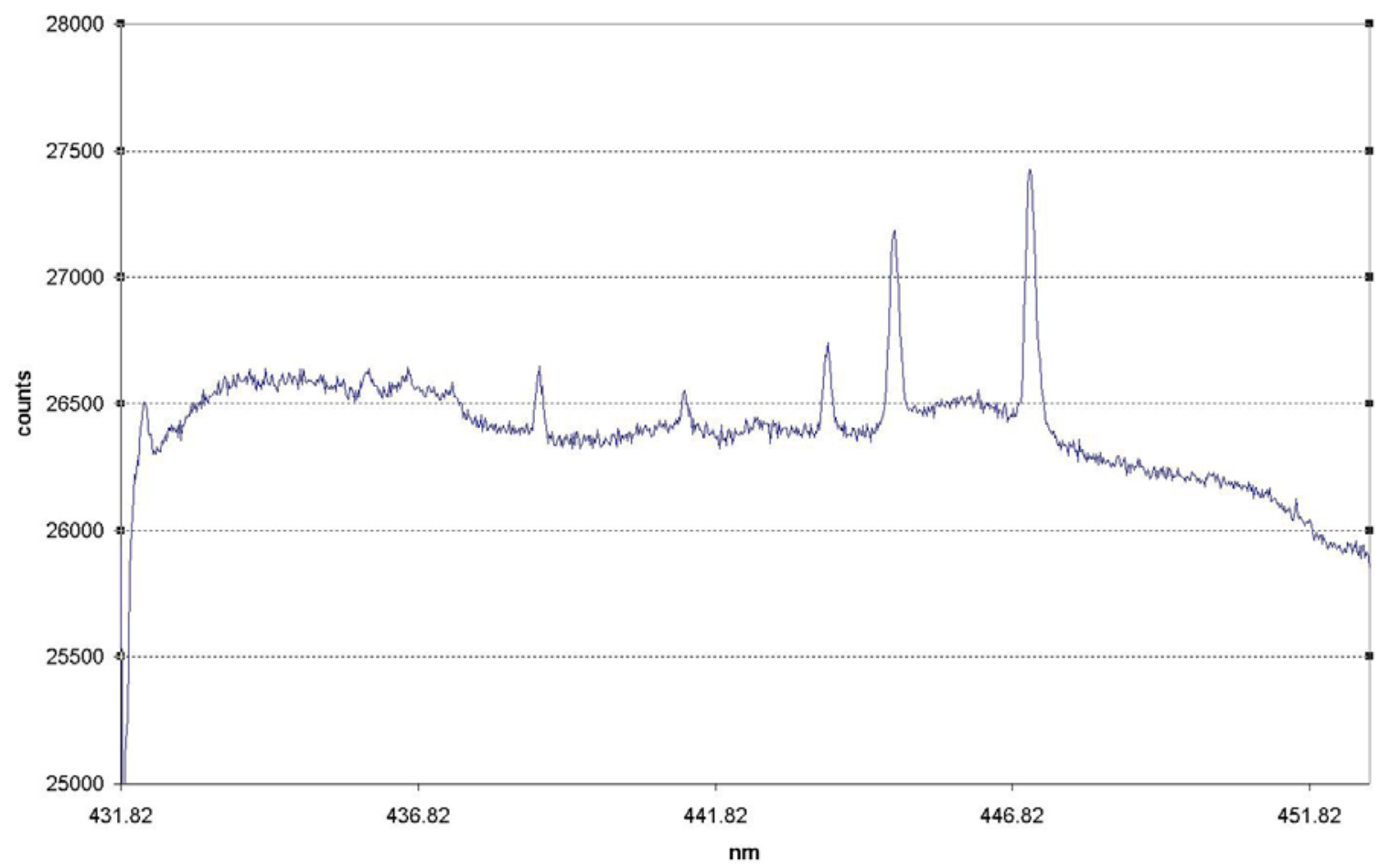

Figure C. 5 - 100 shot average of Mix 4 


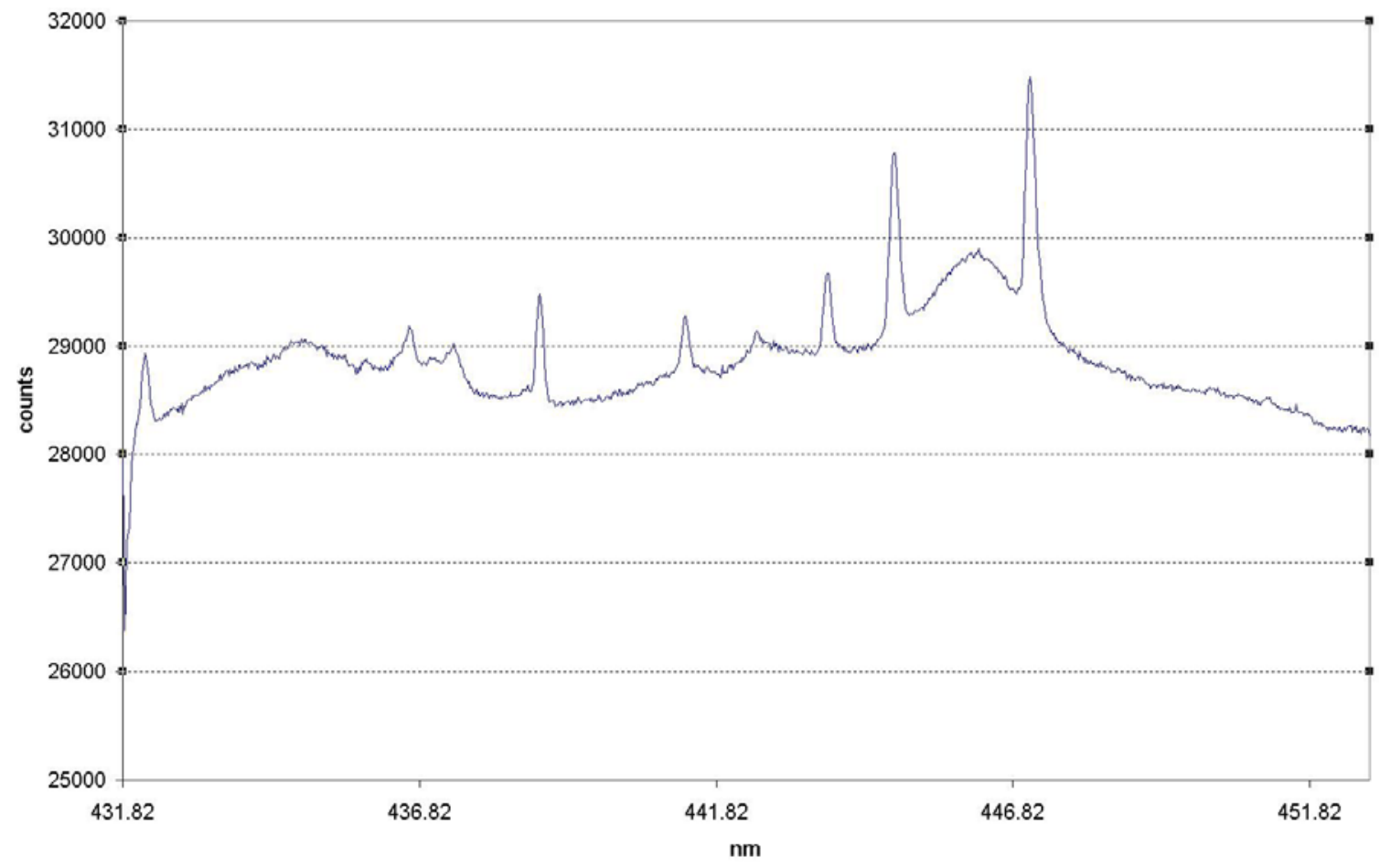

Figure C. 6 - 100 shot average of Mix 5

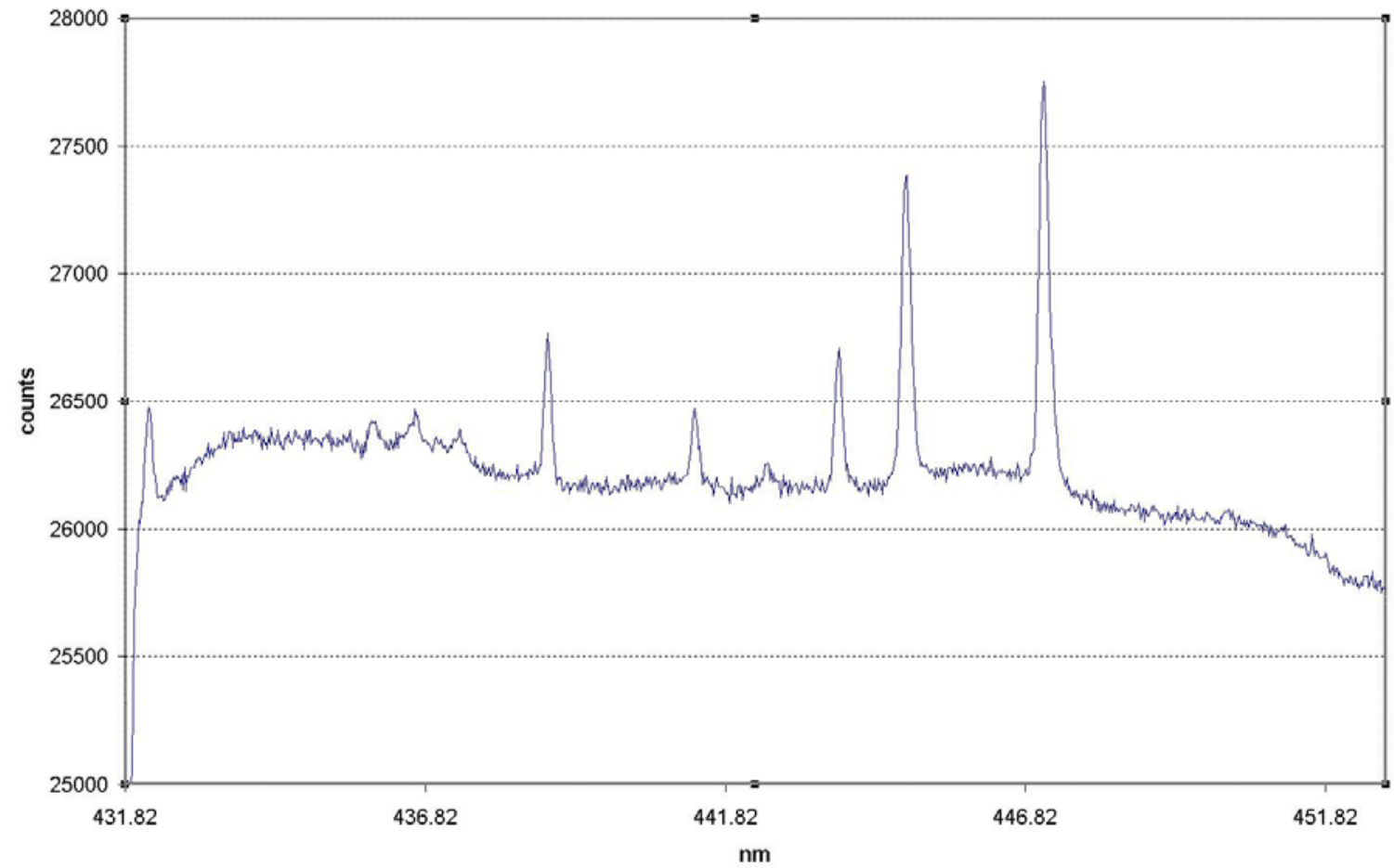

Figure C. 7 - 100 shot average of Mix 6 
MIX 7

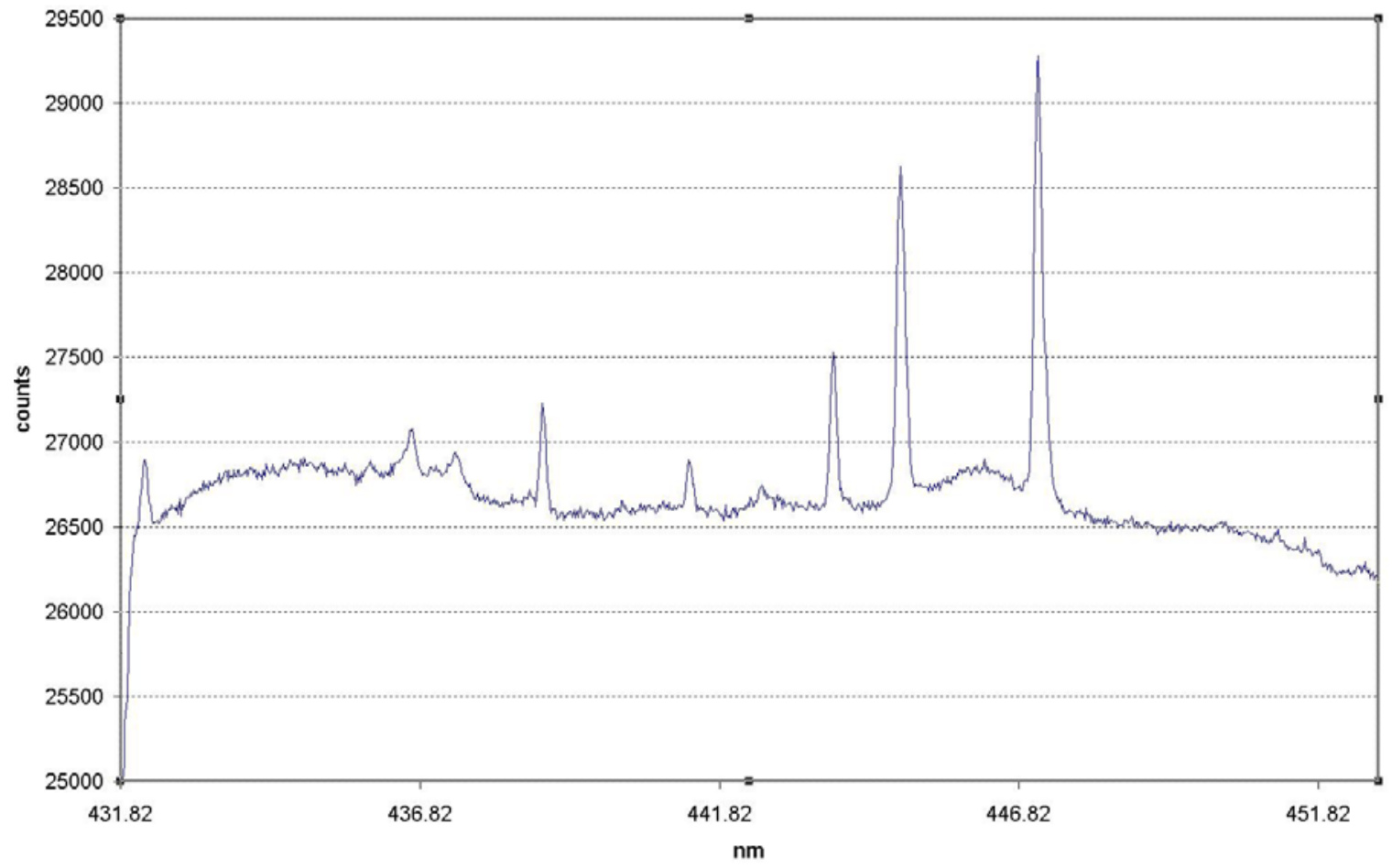

Figure C. 8 - 100 shot average of Mix 7

MIX 8

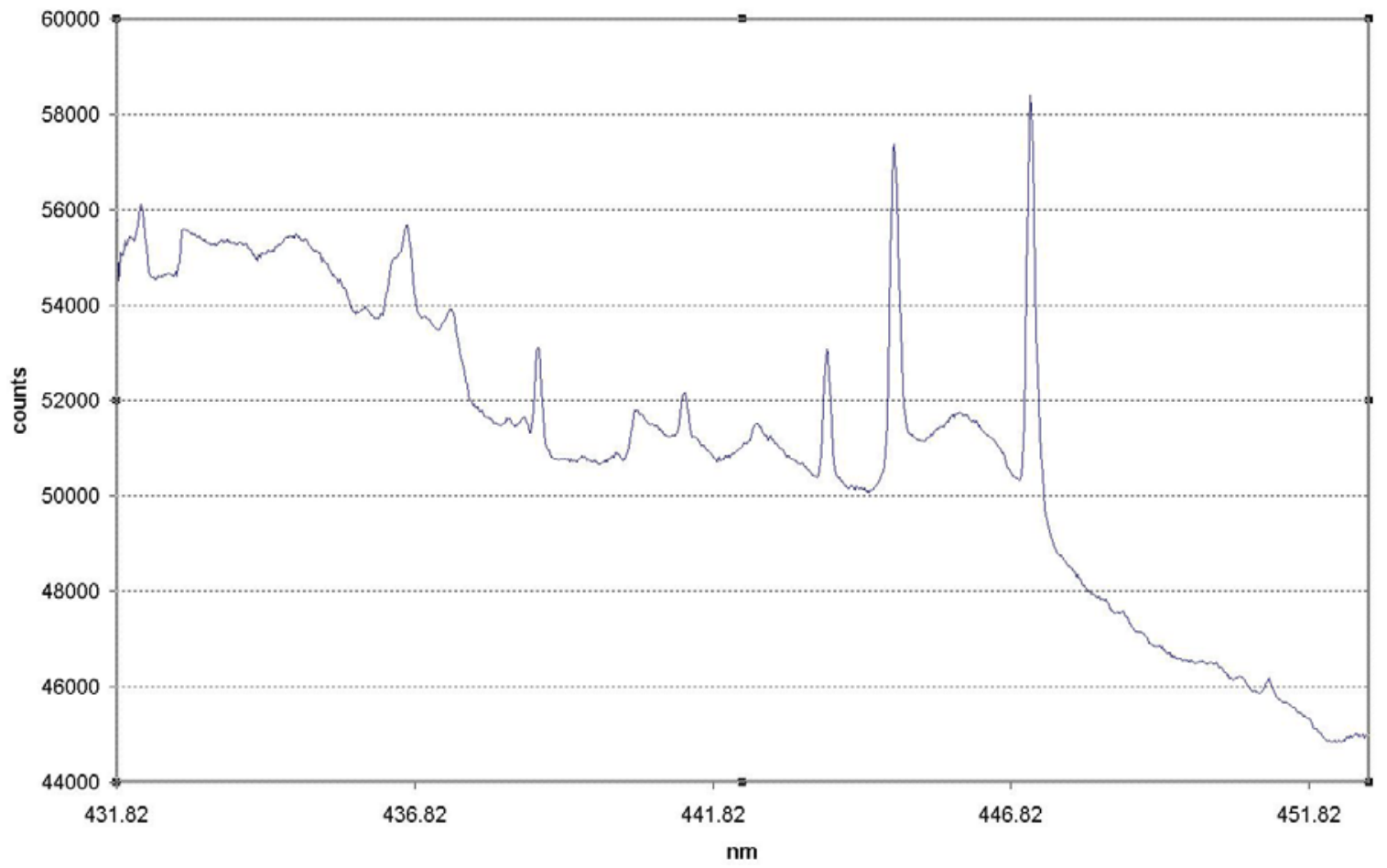

Figure C. 9 - 100 shot average of Mix 8 
$\operatorname{MIX} 9$

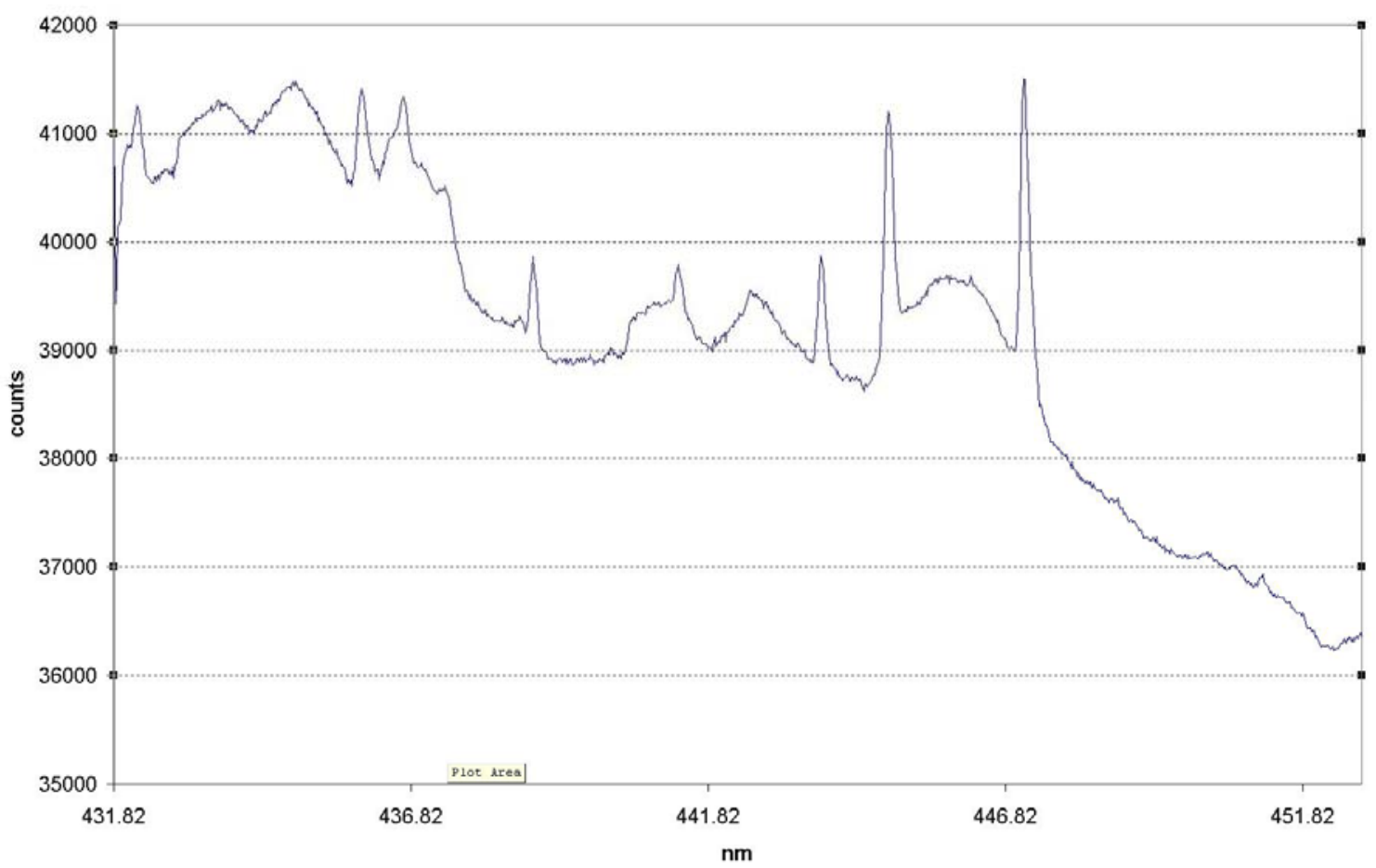

Figure C. 10 - 100 shot average of Mix 9

MIX 10

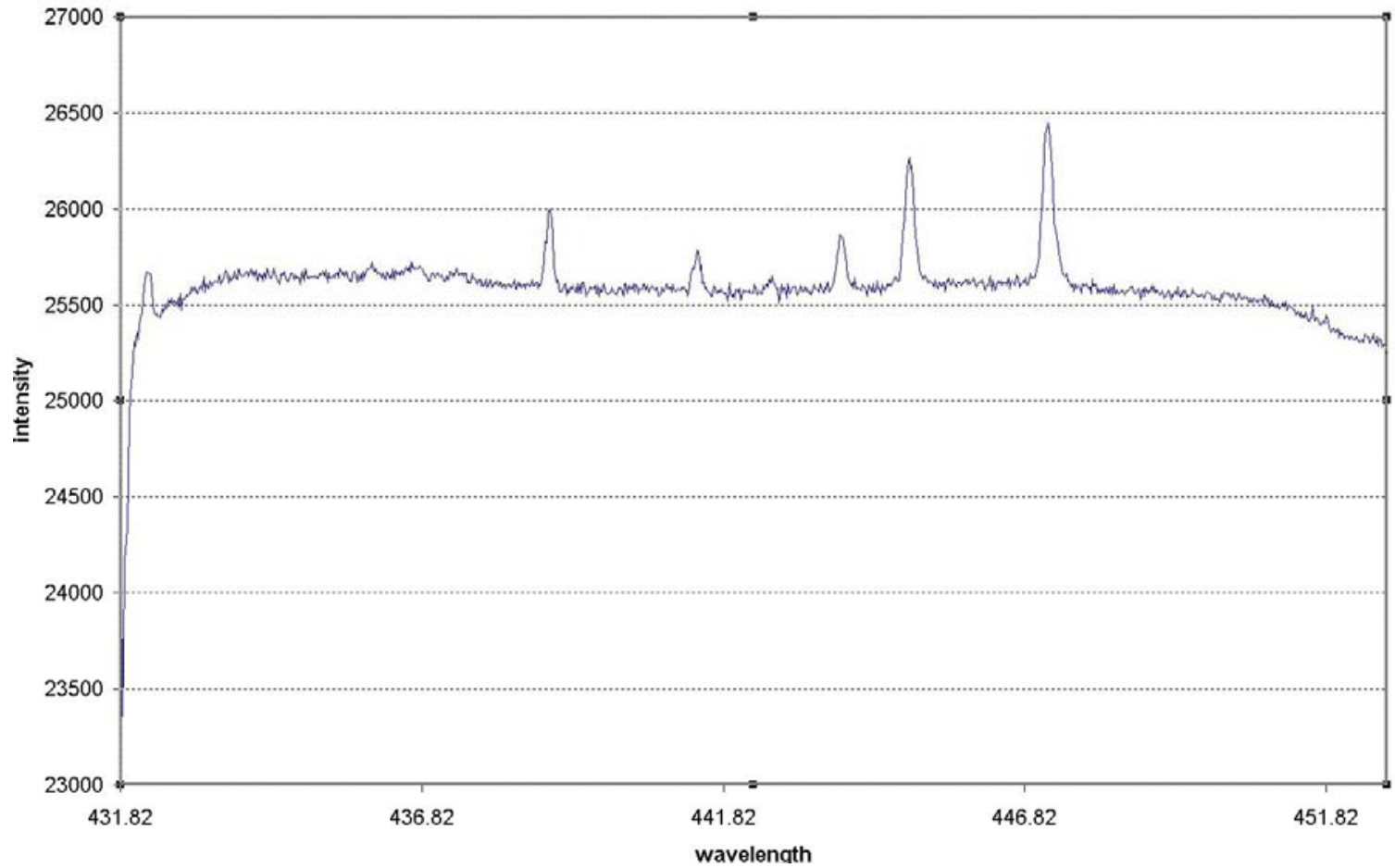

Figure C. 11 - 100 shot average of Mix 10 


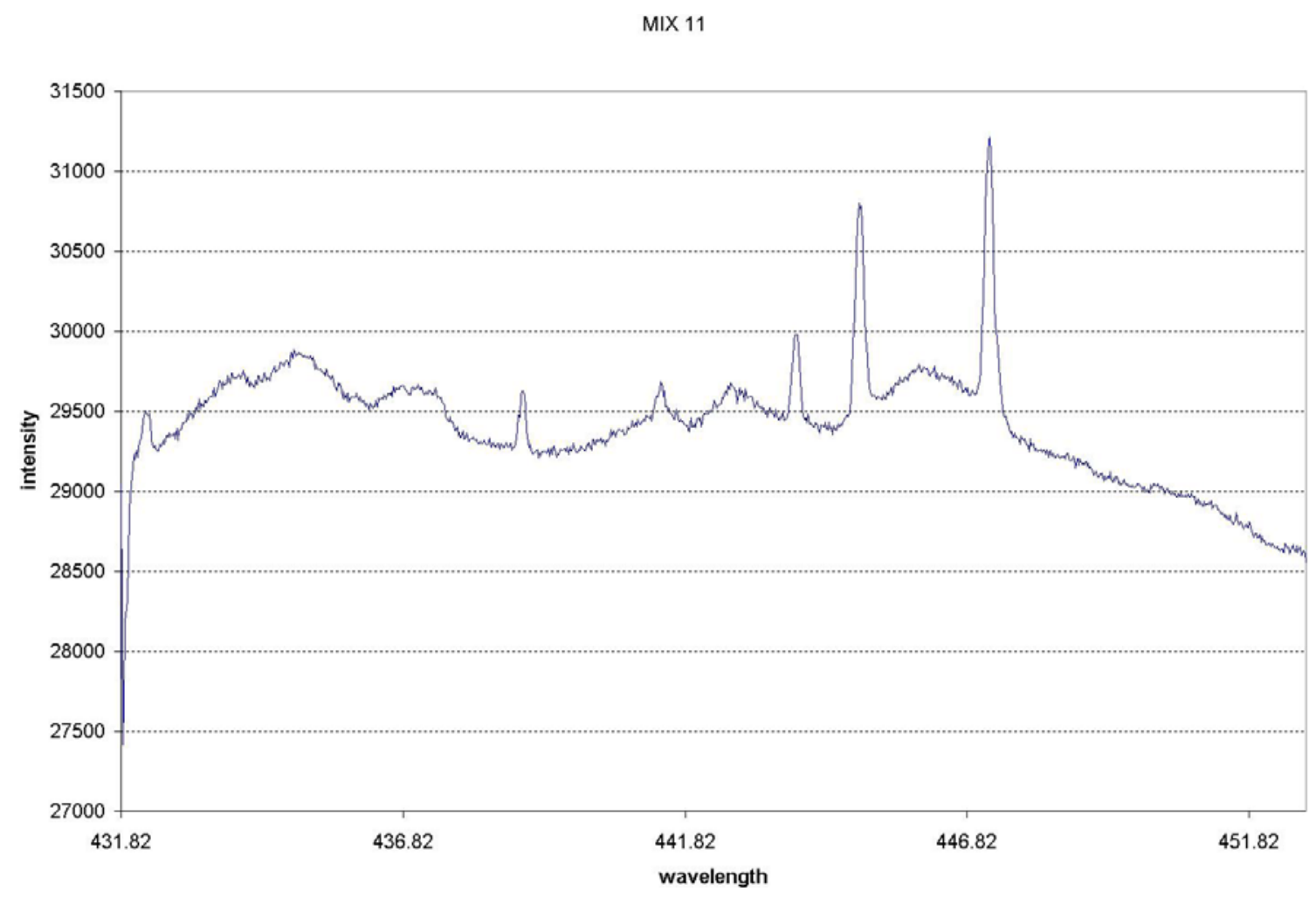

Figure C. 12 - 100 shot average of Mix 11

Synthetic mixtures 1 through 9 were used in regular testing. Synthetic mixtures 10 and 11 were used to test discrepancies of iron in samples. 


\section{APPENDIX D: DYNAMIC LIBS DATA SETS}

This appendix shows raw data sets from dynamic LIBS. Dynamic LIBS was tested on pure iron, a mix of iron and mercury, and synthetic mixtures 1, and 3-9. Mixture 2 was not included because it was destroyed during testing, and mixtures 10 and 11 were not included because they were specifically made for testing discrepancies of iron in the samples. Because the process for dynamic LIBS was not synchronized, a "brute force” method was used, subjecting the pellets to LIBS testing until a signal was obtained. Of all the dynamic data of the synthetic coal mixtures, only 14 individual shots were selected to appear in this appendix, because these shots show the dynamic data of all elements of interest within the experiment. When reading the dynamic LIBS data, the continuum emission will be at the bottom due to the positioning of the laser spark and the rotation of the mirror. The elemental emission lines observed in the dynamic LIBS experiment are the same lines observed in the static LIBS experiment.

\begin{tabular}{|l|l|l|l|l|l|l|l|l|}
\hline Synthetic Mix & Mix1 & Mix3 & Mix4 & Mix5 & Mix6 & Mix7 & Mix8 & Mix9 \\
\hline Dynamic Shots & 72 & 19 & 16 & 11 & 6 & 20 & 20 & 20 \\
\hline
\end{tabular}

Table D. 1 - Number of Dynamic Shots per Synthetic Mixture

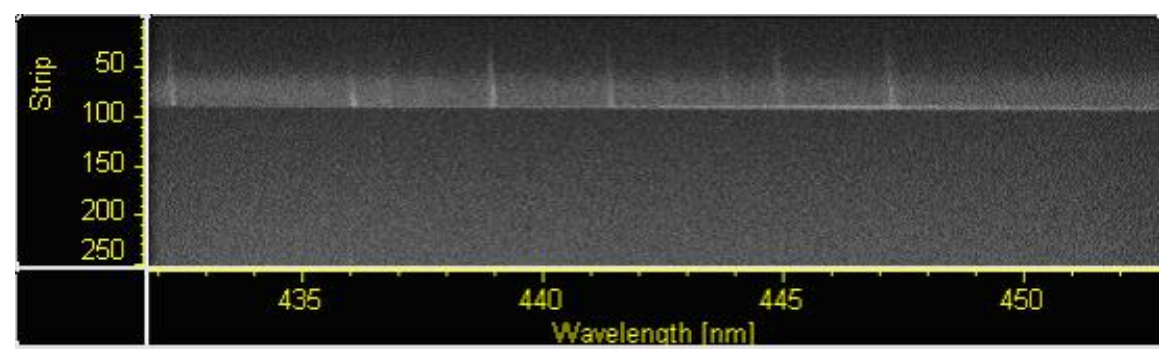

Figure D. 1 - Mix 1 Shot 7 


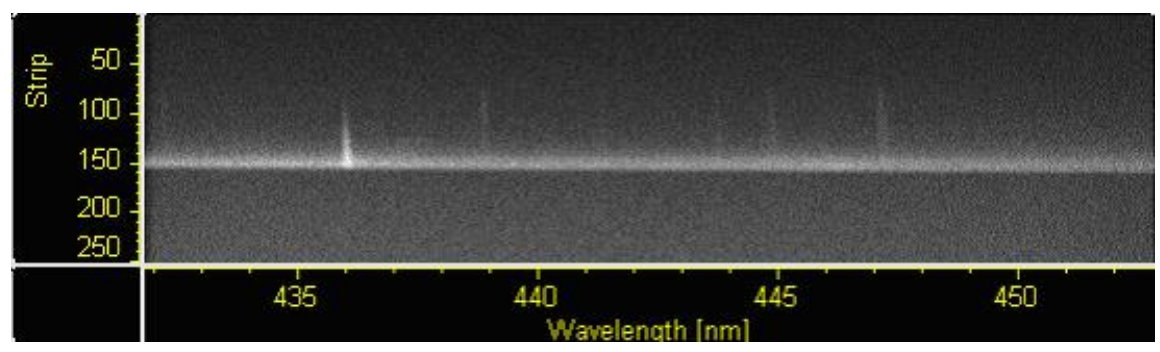

Figure D. 2 - Mix 1 Shot 37

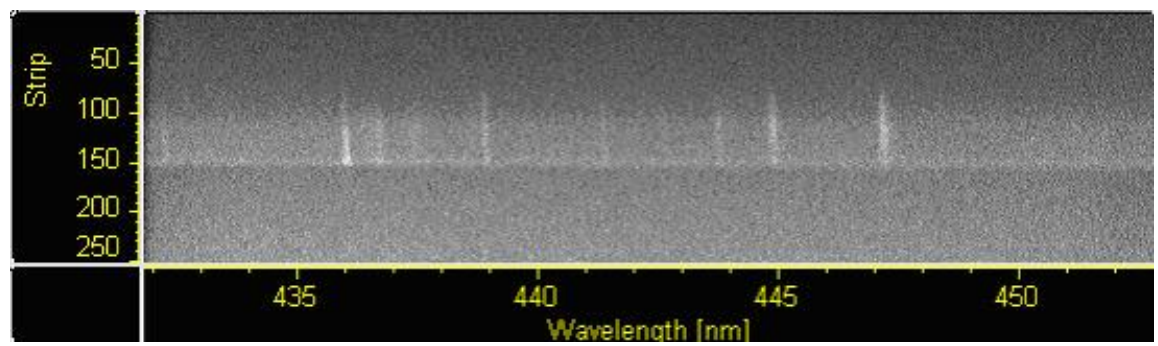

Figure D. 3 - Mix 1Shot 52

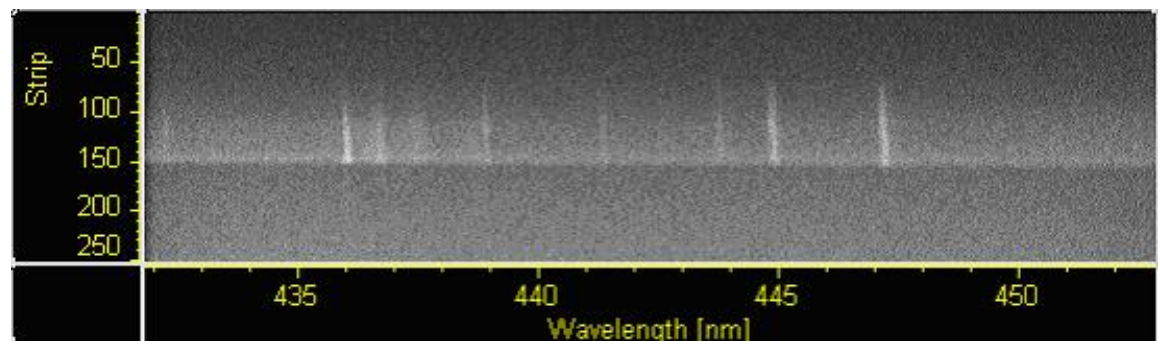

Figure D. 4 - Mix 1 Shot 53

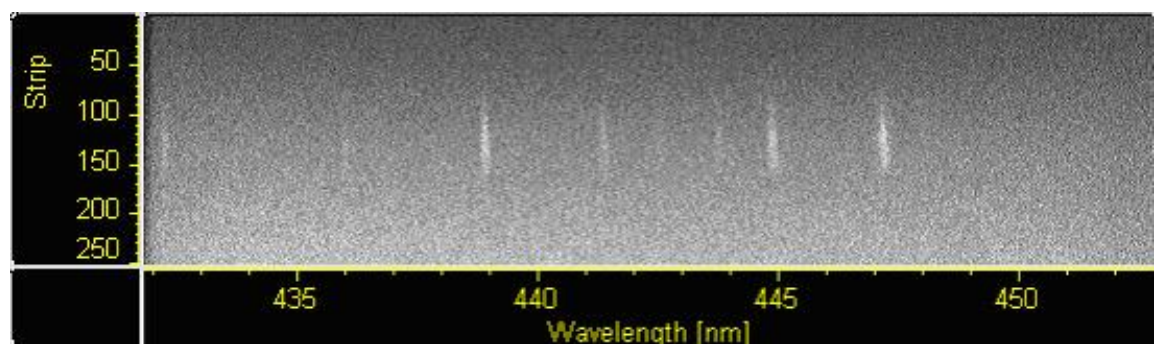

Figure D. 5 - Mix 1 Shot 70 


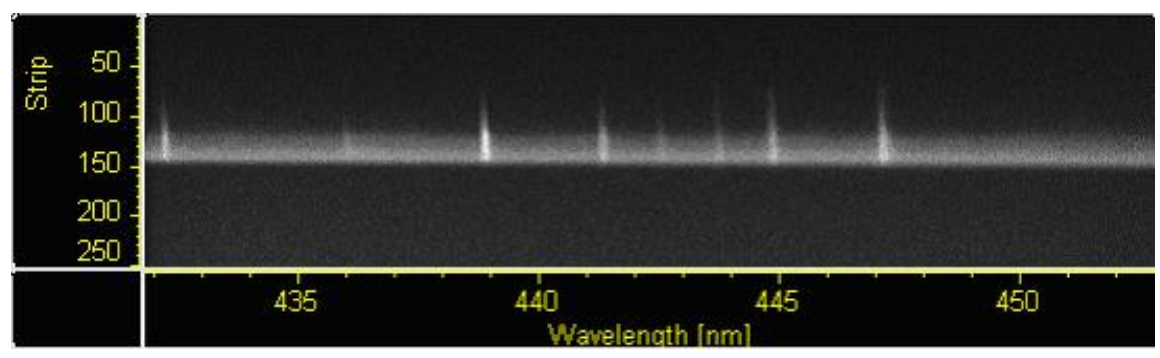

Figure D. 6 - Mix 3 Shot 1

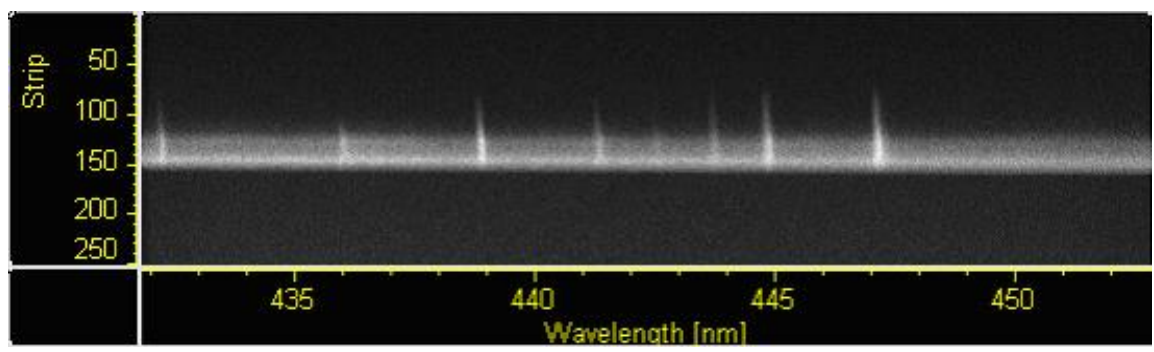

Figure D. 7 - Mix 3 Shot 3

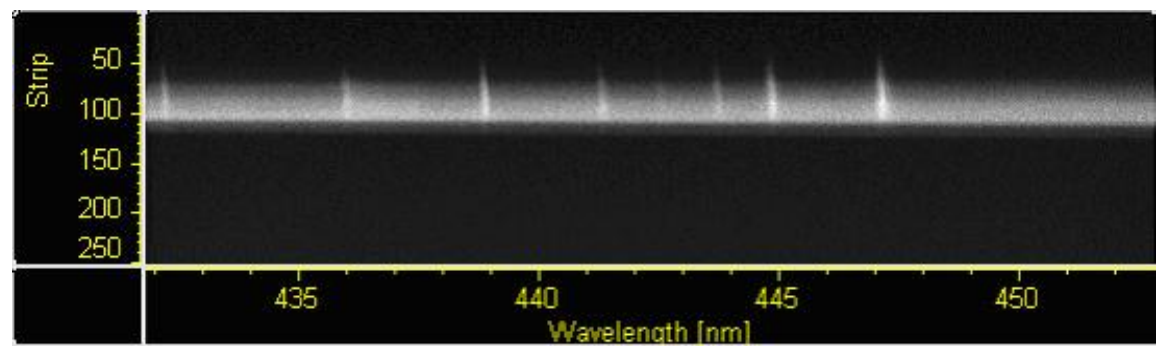

Figure D. 8 - Mix 3 Shot 4

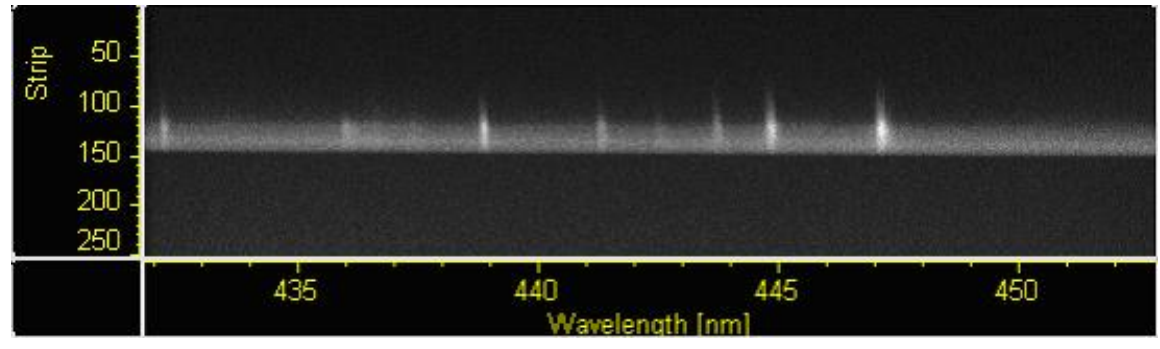

Figure D. 9 - Mix 3 Shot 5 


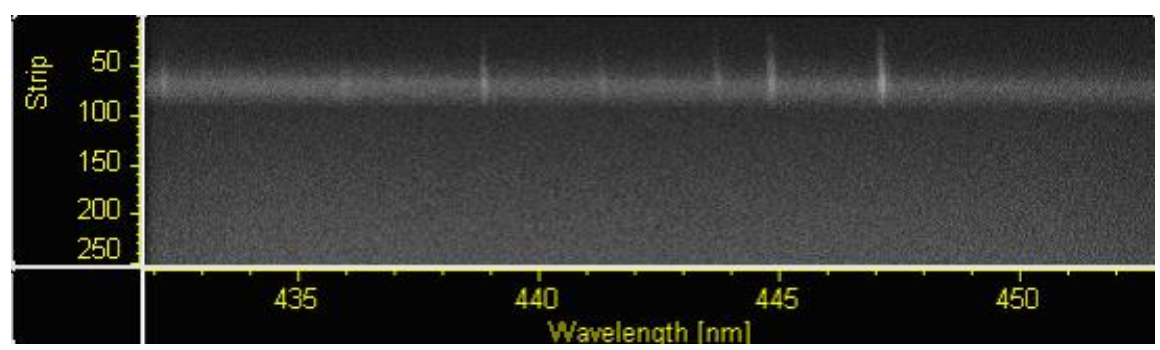

Figure D. 10 - Mix 3 Shot 9

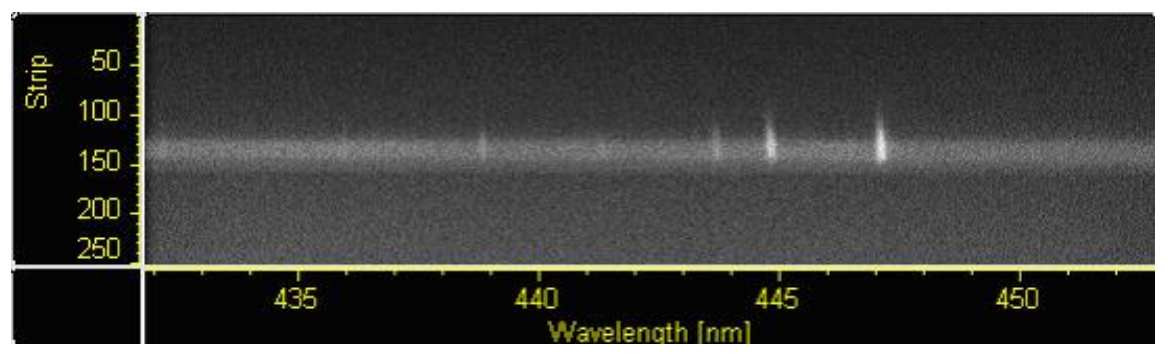

Figure D. 11 - Mix 4 Shot 14

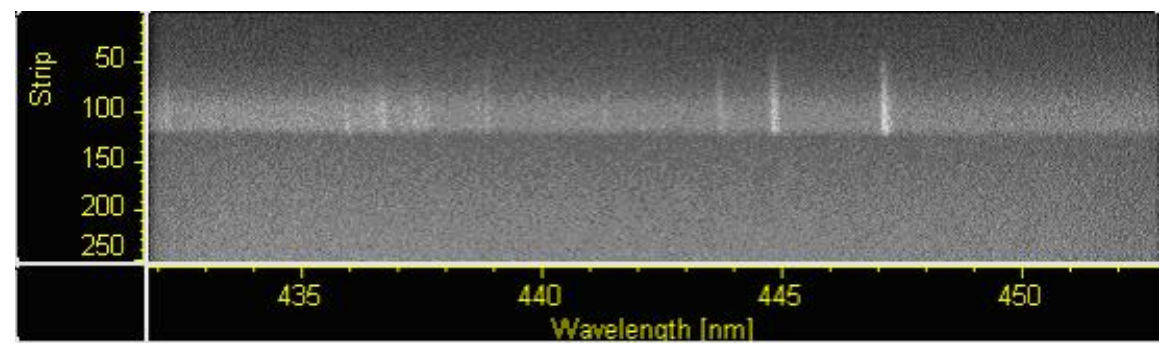

Figure D. 12 - Mix 9 Shot 4

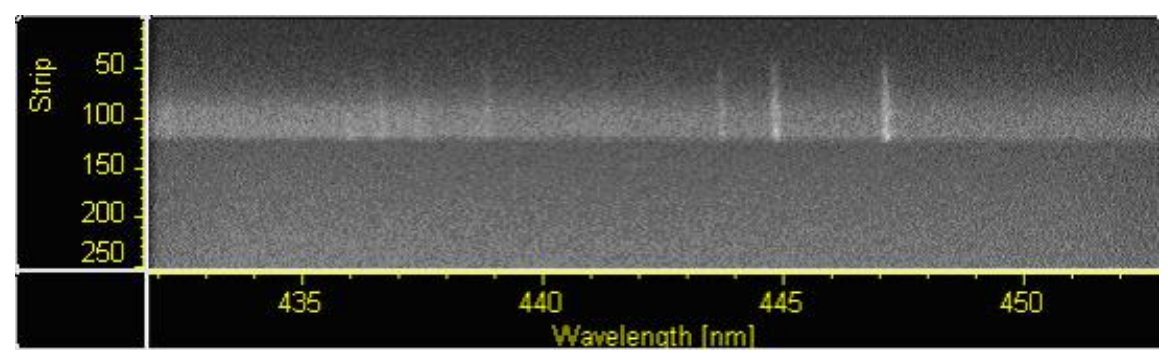

Figure D. 13 - Mix 9 Shot 5 


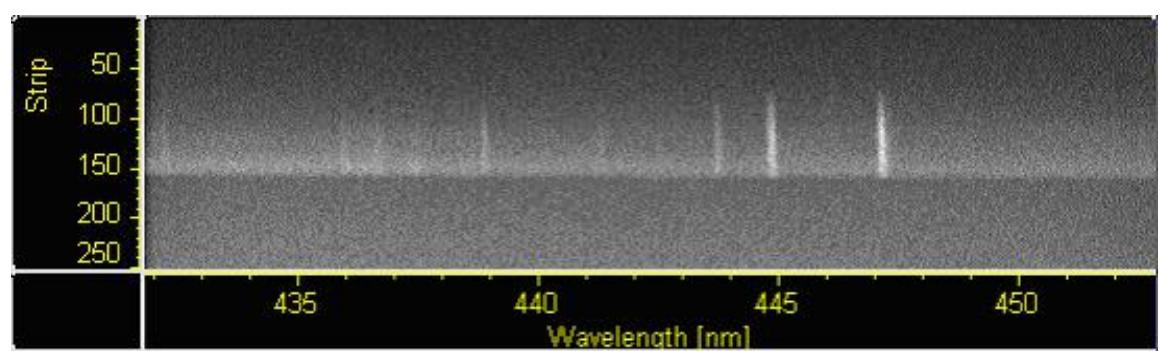

Figure D. 14 - Mix 9 Shot 6

10 dynamic LIBS shots were taken of the iron and mercury mixture. Each shot shows similar temporal characteristics of the emission lines. Notice that the emission lines in this mixture, like the emission lines in the other mixtures, show different lengths along the y-axis. Because of the polygonal scanning mirror, these lengths are a time dependent property of the individual emission lines and share the same time scale as the rest of the dynamic LIBS shots.

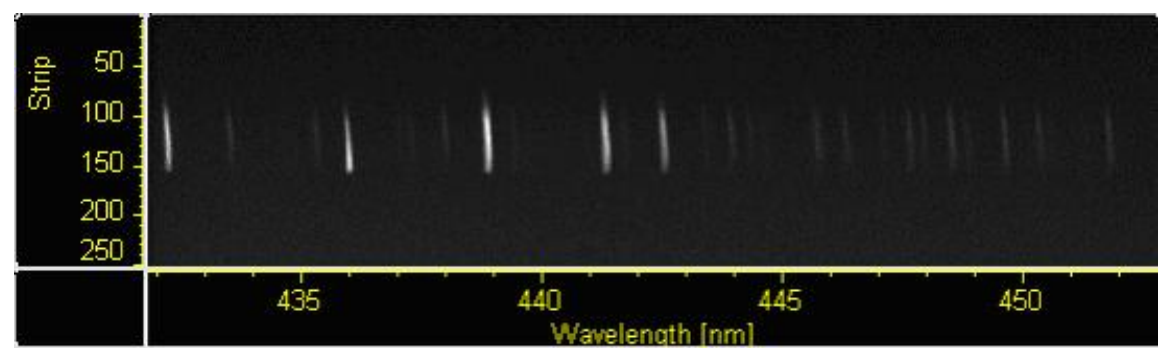

Figure D. 15 -Iron-Mercury Mix Shot 1

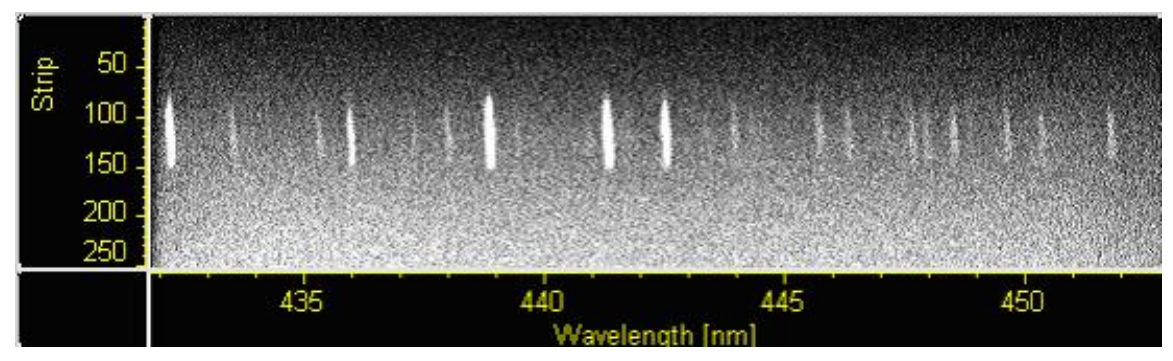

Figure D. 16 -Iron-Mercury Mix Shot 2 


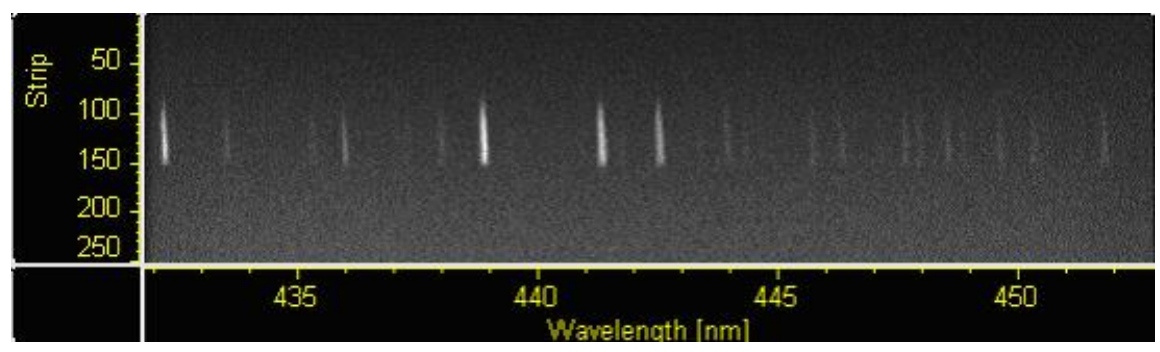

Figure D. 17 -Iron-Mercury Mix Shot 3

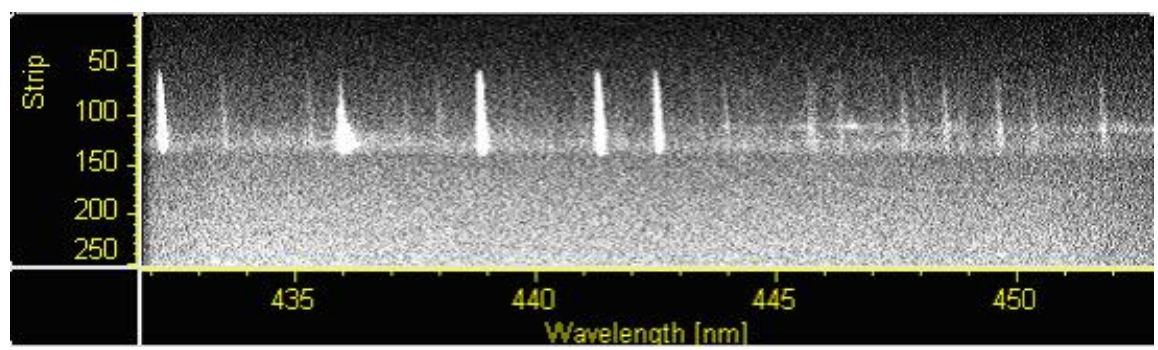

Figure D. 18 -Iron-Mercury Mix Shot 4

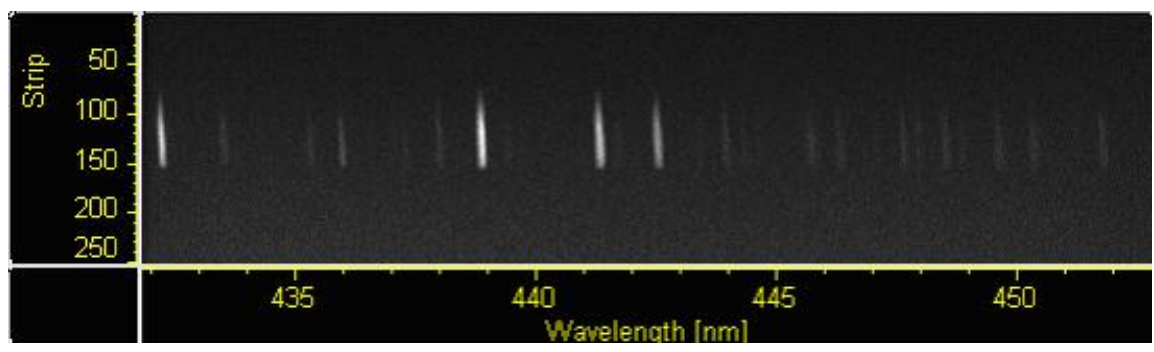

Figure D. 19 -Iron-Mercury Mix Shot 5

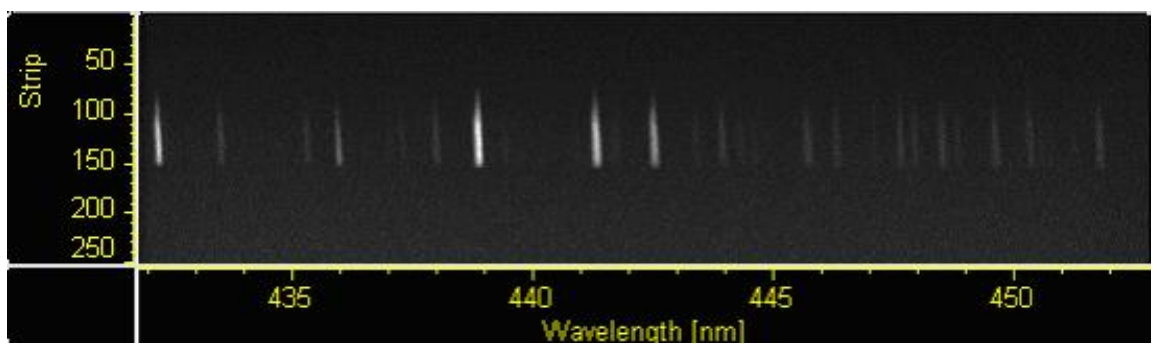

Figure D. 20 -Iron-Mercury Mix Shot 6 


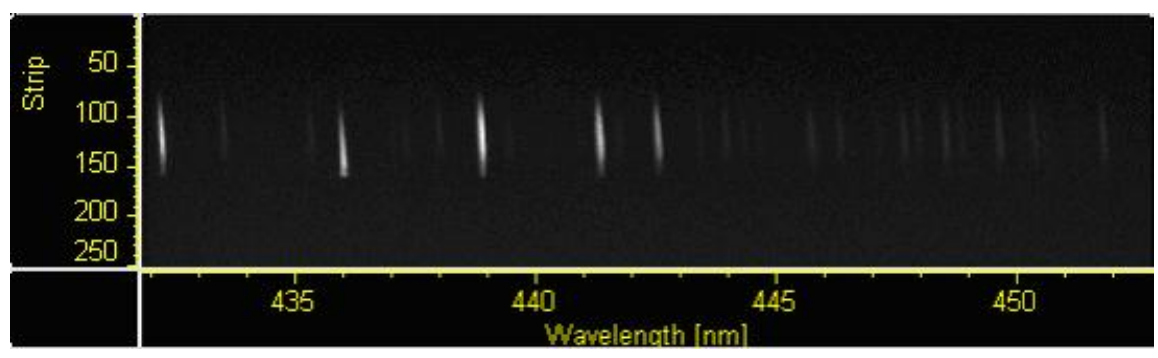

Figure D. 21 -Iron-Mercury Mix Shot 7

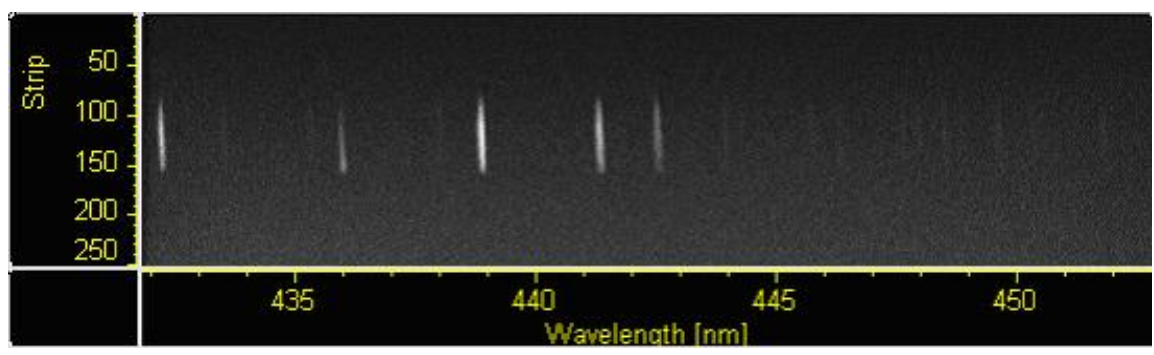

Figure D. 22 -Iron-Mercury Mix Shot 8

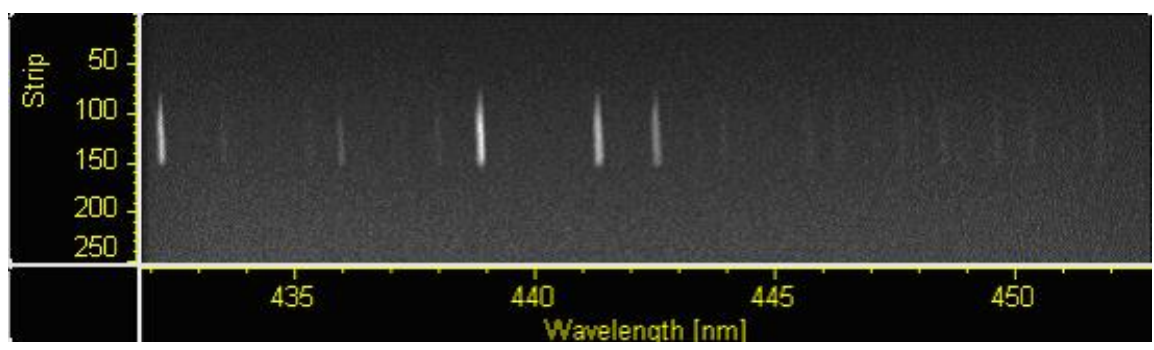

Figure D. 23 -Iron-Mercury Mix Shot 9

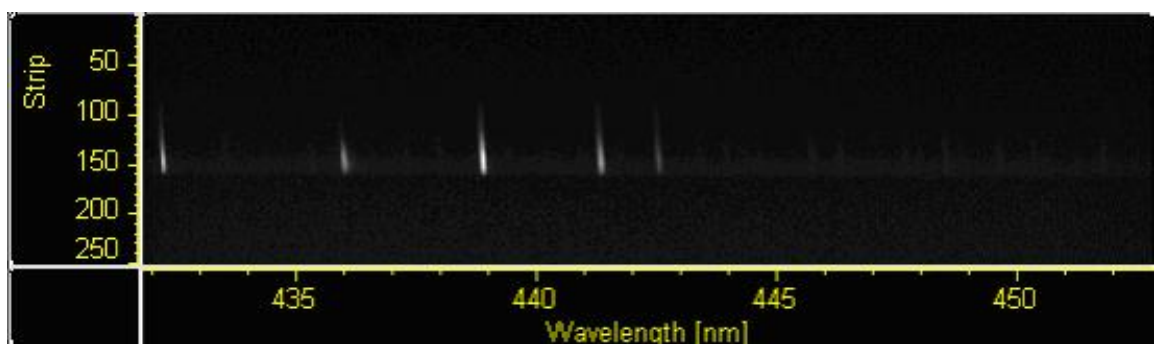

Figure D. 24 -Iron-Mercury Mix Shot 10 